\title{
Inhomogeneous superconductivity in condensed matter and QCD
}

\author{
Roberto Casalbuoni* \\ TH-Division, CERN, CH-1211 Geneva 23, Switzerland \\ Giuseppe Nardulli ${ }^{\dagger}$ \\ Department of Physics, University of Bari, I-70126 Bari, Italy \\ and INFN, Bari, Italy
}

(Published 26 February 2004)

\begin{abstract}
Inhomogeneous superconductivity arises when the species participating in the pairing phenomenon have different Fermi surfaces with a large enough separation. In these conditions it could be more favorable for each of the pairing fermions to stay close to its Fermi surface and, unlike the usual BCS state, for the Cooper pair to have a nonzero total momentum. For this reason, in this state the gap varies in space, the ground state is inhomogeneous, and a crystalline structure might be formed. This situation was considered for the first time by Fulde and Ferrell (1964) and Larkin and Ovchinnikov (1964), after whom the corresponding state is called the LOFF state. The spontaneous breaking of the space symmetries in the vacuum state is a characteristic feature of this phase and is associated with the presence of long-wavelength excitations of zero mass. The situation described here is of interest both in solid-state and in elementary-particle physics, in particular in quantum chromodynamics at high density and low temperature. This review presents the theoretical approach to the LOFF state and its phenomenological applications using the language of the effective field theories.
\end{abstract}

\section{CONTENTS}

I. Introduction

II. The General Setting

A. Nambu-Gor'kov equations

B. Homogeneous superconductors and their phase diagram

C. Gap equation for anisotropic superconductor: One plane wave (Fulde-Ferrell state)

III. Ginzburg-Landau Approximation

A. Gap equation in the Ginzburg-Landau approach $\quad 275$

B. Grand potential

C. Crystalline structures

1. One plane wave

2. Generic crystals

3. Two plane waves

4. Other structures

D. The LOFF phase around the tricritical point; Larkin-Ovchinnikov subspace

IV. Superconductivity in Quantum Chromodynamics

A. High-density effective-field theory

B. Color-flavor-locked phase

C. Two-flavor superconducting phase

D. LOFF phase in QCD

E. One-gluon exchange approximation

F. Mass effects

V. Phonon and Gluon Effective Lagrangians
A. Effective Lagrangian for the LOFF phase
B. One-plane-wave structure
C. Parameters of the phonon effective Lagrangian: one plane wave
D. Cubic structure

263

266

266

268

273

275

277

277

278

278

279

280

284

286

287

288

289

292

293

293

294

295

297

300

*On leave from the Department of Physics of the University of Florence, 50019, Florence, Italy. Electronic address: casalbuoni@fi.infn.it

${ }^{\dagger}$ Electronic address: giuseppe.nardulli@ba.infn.it
E. Parameters of the phonon effective Lagrangian: cubic crystal

F. Gluon dynamics in the LOFF phase $\quad 303$

1. One-plane-wave structure 303

2. Cubic structure 304

VI. Inhomogeneous Superconductivity in Condensed Matter, Nuclear Physics, and Astrophysics 305

A. Type-I superconductors 305

B. "Clean" and strongly type-II superconductors 306

C. Heavy-fermion superconductors 307

D. Two-dimensional, quasi-two-dimensional, and organic superconductors 307

E. Future developments 309

F. LOFF phase in nuclear physics 309

G. Why color LOFF superconductivity could exist in pulsars

H. Astrophysical implications of the QCD LOFF phase

VII. Conclusions

Acknowledgments

Appendix A: Calculation of $J$ and $K$

Appendix B: Expansion of $\Pi$ Around the Tricritical Point 316

References

\section{INTRODUCTION}

The story of superconductivity is one of the most fascinating chapters of modern physics. It has had far reaching influence in many different domains of physics and has shown a tremendous capacity for crossfertilization, to say nothing of its numerous technological applications. This review is devoted to a less-wellknown aspect of this story, inhomogeneous superconductivity. Before giving a more accurate definition of this phenomenon, let us briefly sketch the historical path leading to it. This path began with KamerlinghOnnes (1911), who discovered that the electrical resistance of various metals, e.g., mercury, lead, tin, and 
many others, disappeared when the temperature was lowered below some critical value $T_{c}$. The actual values of $T_{c}$ varied with the metal, but they were all of the order of a few degrees $\mathrm{K}$, or at most of the order of tenths of a degree K. Subsequently, perfect diamagnetism in superconductors was discovered (Meissner and Ochsenfeld, 1933). This property implied not only that magnetic fields are excluded from superconductors, but also that any field originally present in the metal is expelled from it when the temperature is lowered below its critical value. These two features were captured in the equations proposed by the brothers F. London and $\mathrm{H}$. London (1935), who first realized the quantum character of the phenomenon.

In the decade starting in 1950, there were two major theoretical breakthroughs. First, Ginzburg and Landau (1950) created a theory describing the transition between the superconducting and the normal phases. It should be noted that, when it appeared, the GinzburgLandau theory looked rather phenomenological and was not really appreciated in the western literature. Seven years later Bardeen, Cooper, and Schrieffer (BCS) created the microscopic theory that bears their initials (Bardeen et al., 1957). Their theory was based on the fundamental theorem of Cooper (1956), which states that, for a system of many electrons at small $T$, any weak attraction, no matter how small it is, can bind two electrons together, forming the so-called Cooper pair. Subsequently Gor'kov (1959) realized that the Ginzburg-Landau theory was equivalent to the BCS theory around the critical point, and this result vindicated the Ginzburg-Landau theory as a masterpiece in physics. Furthermore, Gor'kov proved that the fundamental quantities of the two theories, i.e., the BCS parameter gap $\Delta$ and the Ginzburg-Landau wave function $\psi$ were related by a proportionality constant, so that $\psi$ could be thought of as the Cooper-pair wave function in the center-of-mass frame. In a sense, the GinzburgLandau theory was the prototype of the modern effective theories. In spite of its limitation to the phase transition, it has a larger field of application, as shown, for example, by its use in the inhomogeneous cases, when the gap is not uniform in space.

Another remarkable advance in these years was Abrikosov's theory of type-II superconductors (Abrikosov, 1957), a class of superconductor allowing penetration of the magnetic field, within certain critical values.

The exciting work in superconductivity inspired related work in the field of elementary-particle physics. Two pioneering papers (Nambu and Jona-Lasinio, 1961a, 1961b) introduced the idea of generating elementary-particle masses through the mechanism of dynamical symmetry breaking suggested by superconductivity. This idea was so fruitful that it eventually became a crucial ingredient of the standard model of elementary particles, in which the masses are generated by the formation of the Higgs condensate much in the same way as superconductivity originates from the presence of a gap. Furthermore, the Meissner effect, which is characterized by a penetration length, is the origin, in the elementary-particle-physics language, of the masses of the gauge-vector bosons. These masses are nothing but the inverse of the penetration length.

With the advent of quantum chromodynamics (QCD) it was early realized that at high density, due to the property of asymptotic freedom (Gross and Wilczek, 1973; Politzer, 1973) and to the existence of an attractive channel in the color interaction, diquark condensates might be formed (Collins and Perry, 1975; Barrois, 1977; Frautschi, 1978; Bailin and Love, 1984). Since these condensates break the color gauge symmetry, the subject took the name of color superconductivity. In the last few years this has become a very active field of research; these developments are reviewed by Hsu (2000), Alford (2001), Hong (2001), Rajagopal and Wilczek (2001), and Nardulli (2002a). It should also be noted that color superconductivity might have implications for astrophysics because in some compact stars, e.g., pulsars, the baryon densities necessary for color superconductivity are probably reached.

Superconductivity in metals was the subject of another breakthrough in the 1980s with the discovery of high- $T_{c}$ superconductors. The main subject of this review, however, is a different and separate development of superconductivity, which took place in 1964. It originates in high-field superconductors where a strong magnetic field, coupled to the spins of the conduction electrons, gives rise to a separation of the Fermi surfaces corresponding to electrons with opposite spins. If the separation is too high, the pairing is destroyed and there is a transition (first-order at small temperature) from the superconducting state to the normal one. In independent papers, Larkin and Ovchinnikov (1964) and Fulde and Ferrell (1964) showed that a new state could be formed, close to the transition line. This state, which hereafter will be called the $\mathrm{LOFF}^{1}$ state or LOFF phase, has the feature of exhibiting an order parameter, or a gap, which is not a constant, but has a space variation whose typical wavelength is of the order of the inverse of the difference in the Fermi energies of the pairing electrons. The space modulation of the gap arises because the electron pair has nonzero total momentum, and it is a rather peculiar phenomenon that leads to the possibility of a nonuniform or anisotropic ground state, breaking translational and rotational symmetries. It has also been conjectured that the typical inhomogeneous ground state might have a periodic or, in other words, a crystalline structure. For this reason, this phenomenon is also known as inhomogeneous, anisotropic, or crystalline superconductivity.

Inhomogeneous superconductivity in metals has been the object of intense experimental investigation, especially in the last decade. For reasons to be discussed below, the experimental research has been aimed at rather unconventional superconductors, such as heavyfermion superconductors, quasi-two-dimensional lay-

\footnotetext{
${ }^{1}$ In the literature the LOFF state is also known as the FFLO state.
} 
ered organic superconductors, or high- $T_{c}$ superconductors. While different from the original LOFF proposal, these investigations still aim at attaining a superconducting state characterized by nonzero total momentum of the Cooper pair and space modulation of its wave function. At the moment they represent the main possibility of discovering the LOFF state in condensed-matter physics.

Quite recently, it has also been realized that at moderate density the mass difference between the strange, up, and down quarks at the weak equilibrium and/or color and electric neutrality lead to a difference in the Fermi momenta, which, in principle, renders the LOFF state possible in color superconductivity (Alford et al., 2001b). The same authors have pointed out that this phenomenon might have some relevance in explaining the sudden variations (glitches) in the rotation periods of pulsars.

We should also point out that other possibilities have been explored in the literature allowing pairing between quarks with different Fermi surfaces. The first possibility, the deformed-Fermi-sphere superconductor, was considered by Muther and Sedrakian (2002). The idea is that Fermi spheres are deformed by the interactions in such a way that they intersect, which allows pairing in a region close to the intersection. This makes the pairing similar to that in the LOFF phase (although there is no breaking of translational invariance) but with a large energy cost due to the deformation. For this reason, one may suspect that the deformed-Fermi-sphere phase has a larger free energy than the LOFF phase. The second possibility is "breached-pair" color superconductivity (Gubankova et al., 2003; Liu and Wilczek, 2003). When the Fermi sphere of the heavy quarks is smaller than that of the light fermions, heavy quarks move from their own surface to momenta of the order of the Fermi momentum of the light sphere, thus allowing Cooper-pair formation. It turns out that this phase is stable depending on whether one is at a fixed number density or at a fixed chemical potential. An energy-free comparison with the LOFF case has not yet been done. These two possibilities are certainly very interesting. However, much study is still necessary in order to reach a firm conclusion about their physical realization. Therefore we shall not discuss them any further in this review.

The main aim of this review is to present the ideas and methods of the two main paths to inhomogeneous superconductivity, i.e., condensed matter and QCD. Our approach will be mainly theoretical, and the discussion of phenomenological consequences will be limited, first because we lack the necessary skills, and second because the theory of LOFF superconductivity is currently more advanced than experiment, and its main phenomenological implications belong to the future. For this reason, we shall devote more space to the theoretical foundations of inhomogeneous superconductivity and will present only a summary of the experimental results. We shall attempt to show the similarities of different physical situations and to present as unified a formalism as possible. This is not only to prove once again the cross- fertilization power of superconductivity, but also to expose experts in the two fields to results that may be easily transferrable from one sector to the other. Moreover, by presenting the LOFF phenomenon in a unified formalism, this review can contribute, we hope, to establishing a common language. To this end, we discuss the LOFF state both in solid-state and in QCD physics, starting with the Nambu-Gor'kov equations. From the solid-state side they will be derived by the effective theory of the relevant degrees of freedom at the Fermi surface, and from the QCD sector by the so-called highdensity effective theory which, as we shall see, leads to equations of motion that coincide with the NambuGor'kov equations. In this way we establish a reference that allows us to switch easily from one field to the other.

The plan of this review is as follows. In Sec. II we begin by describing the general formalism based on the Nambu-Gor'kov equations (Gor'kov, 1959; Nambu, 1960). As shown by Polchinski (1993) using the renormalization-group approach, excitations at the Fermi surface can be described by an effective-field theory. Its equations of motion are exactly the NambuGor'kov equations of ordinary (homogeneous) superconductivity. We shall then apply this formalism to fermions with different Fermi surfaces. The difference can be due to a magnetic field producing an energy splitting between spin-up and spin-down electrons, or, as in QCD, to a difference in the chemical potential originating from weak equilibrium, color and electric neutrality, or a difference in mass between the pairing fermions. We shall discuss the circumstances leading, in these cases, to inhomogeneous superconductivity. The Ginzburg-Landau expansion can be used, as already mentioned, for the description of the inhomogeneous phase. It will be discussed in Sec. III, both at zero temperature and close to the tricritical point. The $T=0$ case is more interesting for QCD applications, while the finite-temperature case might be relevant in condensed matter. In Sec. IV we switch to QCD. We shall first give a brief introduction to color superconductivity and then a description of the effective Lagrangian for quarks at zero temperature close to the Fermi surface. We shall also discuss more specifically the LOFF case for QCD with two massless flavors. Since in the LOFF phase both translational and rotational symmetries are spontaneously broken, the Goldstone theorem requires the presence in the physical spectrum of long-wavelength, gapless excitations (phonons). In Sec. V we discuss the phonon effective Lagrangians for two crystalline structures, the single plane wave and the cubic structure. We limit our presentation to the QCD case, though the presence of these excitations is obviously general. We also discuss gluon propagation inside these two crystalline media. In Sec. VI we shall discuss the possible phenomenological applications of the LOFF phase. This discussion will go from strongly type-II superconductors to two-dimensional structures for condensed matter. For hadronic matter we discuss applications both in nuclear physics and in QCD, with particular emphasis on the physics of glitches in pulsars. 
We conclude this introduction by apologizing to the many authors whose work is not reviewed here in depth. Space constraints forced us to sacrifice a more detailed exposition. The extensive bibliography at the end will, we hope, lead the interested reader to the original sources.

\section{THE GENERAL SETTING}

In this section we give a pedagogical introduction to inhomogeneous superconductivity. We begin by reviewing the Nambu-Gor'kov approach to homogeneous superconductivity via a field theory with effective spin-1/2 fields describing quasiparticles. The effective-field theory considers only the relevant degrees of freedom in the limit of small temperatures and high chemical potential; they are the modes in a shell around the Fermi surface. The dominant coupling in this limit is the fourfermion interaction, as first introduced in the BCS model. The dominance of this coupling can be also proved in more modern language by using the renormalization-group approach (Benfatto and Gallavotti, 1990; Polchinski, 1993; Shankar, 1994), which shows that the BCS coupling is marginal and therefore, in the absence of relevant couplings, it can dominate over other irrelevant couplings and produce the phenomenon of superconductivity.

After having derived the Nambu-Gor'kov equations and the gap equation in Sec. II.A, we discuss the case of homogeneous superconductors in Sec. II.B, and analyze its phase diagram. We assume from the very beginning that the two species participating in the Cooper pairing have different chemical potentials, as this is the necessary situation for the LOFF state. In Sec. II.C we discuss anisotropic superconductivity and also show that for appropriate values of the difference in chemical potentials an anisotropic modulated gap $\Delta(\mathbf{r}) \propto \exp (i \mathbf{q} \cdot \mathbf{r})$ leads to a state that is energetically favored in comparison to both the BCS and the normal nonsuperconducting states. This was the state first discussed by Fulde and Ferrell (1964).

\section{A. Nambu-Gor'kov equations}

To start with, we consider, at $T=0$, a fermion liquid formed by two species, $u$ and $d$, having different Fermi energies. In electron superconductivity, as in the original LOFF papers (Fulde and Ferrell, 1964; Larkin and Ovchinnikov, 1964), the species are the electron spin-up and spin-down states, but our formalism is general and will be applied later to the case in which the fermions forming the Cooper pair are two quarks with different flavors. In superconducting materials the difference of chemical potentials can be produced by the presence of paramagnetic impurities. All these cases give rise to an effective exchange interaction that can described by adding the following term to the Hamiltonian:

$$
H_{\text {exch }}=-\delta \mu \psi^{\dagger} \sigma_{3} \psi
$$

In the case of electron superconductivity, $\delta \mu$ is proportional to the magnetic field, and the effect of Eq. (2.1) is to change the chemical potentials of the two species:

$$
\mu_{u}=\mu+\delta \mu, \quad \mu_{d}=\mu-\delta \mu .
$$

Adopting a BCS interaction, the action can be written

$$
\begin{aligned}
& A=A_{0}+A_{B C S}, \\
& A_{0}=\int d t \frac{d \mathbf{p}}{(2 \pi)^{3}} \psi^{\dagger}(\mathbf{p})\left[i \partial_{t}-E(\mathbf{p})+\mu+\delta \mu \sigma_{3}\right] \psi(\mathbf{p}), \\
& A_{B C S}=\frac{g}{2} \int d t \prod_{k=1}^{4} \frac{d \mathbf{p}_{k}}{(2 \pi)^{3}}\left[\psi^{\dagger}\left(\mathbf{p}_{1}\right) \psi\left(\mathbf{p}_{4}\right)\right] \\
& \quad \times\left[\psi^{\dagger}\left(\mathbf{p}_{2}\right) \psi\left(\mathbf{p}_{3}\right)\right] \times(2 \pi)^{3} \delta\left(\mathbf{p}_{1}+\mathbf{p}_{2}-\mathbf{p}_{3}-\mathbf{p}_{4}\right) .
\end{aligned}
$$

Here and below, unless explicitly stated, $\psi(\mathbf{p})$ denotes the three-dimensional (3D) Fourier transform of the Pauli spinor $\psi(\mathbf{r}, t)$, i.e., $\psi(\mathbf{p}) \equiv \psi_{\sigma}(\mathbf{p}, t)$. For nonrelativistic particles the functional dependence of the energy would be $E(\mathbf{p})=\mathbf{p}^{2} / 2 m$, but we prefer to leave it in the more general form of Eq. (2.4).

The BCS interaction (2.5) can be written

$$
A_{B C S}=A_{\text {cond }}+A_{\text {int }} \text {, }
$$

with

$$
\begin{aligned}
& A_{\text {cond }}=-\frac{g}{4} \int d t \prod_{k=1}^{4} \frac{d \mathbf{p}_{k}}{(2 \pi)^{3}}\left[\tilde{\Xi}\left(\mathbf{p}_{3}, \mathbf{p}_{4}\right) \psi^{\dagger}\left(\mathbf{p}_{1}\right) C \psi^{\dagger}\left(\mathbf{p}_{2}\right)\right. \\
& \left.-\widetilde{\Xi} *\left(\mathbf{p}_{1}, \mathbf{p}_{2}\right) \psi\left(\mathbf{p}_{3}\right) C \psi\left(\mathbf{p}_{4}\right)\right](2 \pi)^{3} \\
& \times \delta\left(\mathbf{p}_{1}+\mathbf{p}_{2}-\mathbf{p}_{3}-\mathbf{p}_{4}\right), \\
& A_{\text {int }}=-\frac{g}{4} \int d t \prod_{k=1}^{4} \frac{d \mathbf{p}_{k}}{(2 \pi)^{3}}\left[\psi^{\dagger}\left(\mathbf{p}_{1}\right) C \psi^{\dagger}\left(\mathbf{p}_{2}\right)\right. \\
& \left.+\widetilde{\Xi} *\left(\mathbf{p}_{1}, \mathbf{p}_{2}\right)\right]\left[\psi\left(\mathbf{p}_{3}\right) C \psi\left(\mathbf{p}_{4}\right)-\widetilde{\Xi}\left(\mathbf{p}_{3}, \mathbf{p}_{4}\right)\right] \\
& \times(2 \pi)^{3} \delta\left(\mathbf{p}_{1}+\mathbf{p}_{2}-\mathbf{p}_{3}-\mathbf{p}_{4}\right),
\end{aligned}
$$

where $C=i \sigma_{2}$ and

$$
\widetilde{\Xi}\left(\mathbf{p}, \mathbf{p}^{\prime}\right)=\left\langle\psi(\mathbf{p}) C \psi\left(\mathbf{p}^{\prime}\right)\right\rangle .
$$

In the mean-field approximation the interaction term can be neglected while the gap term $A_{\text {cond }}$ is added to $A_{0}$. Note that the spin-0 condensate $\widetilde{\Xi}\left(\mathbf{p}, \mathbf{p}^{\prime}\right)$ is simply related to the condensate wave function

$$
\Xi(\mathbf{r})=\langle\psi(\mathbf{r}, t) C \psi(\mathbf{r}, t)\rangle
$$

by the formula

$$
\Xi(\mathbf{r})=\int \frac{d \mathbf{p}}{(2 \pi)^{3}} \frac{d \mathbf{p}^{\prime}}{(2 \pi)^{3}} e^{-i\left(\mathbf{p}+\mathbf{p}^{\prime}\right) \cdot \mathbf{r}} \widetilde{\Xi}\left(\mathbf{p}, \mathbf{p}^{\prime}\right) .
$$

In general the condensate wave function can depend on r. Only for homogeneous materials does it not depend on the space coordinates. Therefore in this case $\widetilde{\Xi}\left(\mathbf{p}, \mathbf{p}^{\prime}\right)$ is proportional to $\delta\left(\mathbf{p}+\mathbf{p}^{\prime}\right)$. 
In order to write down the Nambu-Gor'kov equations we define the Nambu-Gor'kov spinor

$$
\chi(\mathbf{p})=\frac{1}{\sqrt{2}}\left(\begin{array}{c}
\psi(\mathbf{p}) \\
\psi^{c}(-\mathbf{p})
\end{array}\right)
$$

where we have introduced the charge-conjugate field

$$
\psi^{c}=C \psi^{\dagger} \text {. }
$$

We also define

$$
\Delta\left(\mathbf{p},-\mathbf{p}^{\prime}\right)=\frac{g}{2} \int \frac{d \mathbf{p}^{\prime \prime}}{(2 \pi)^{6}} \widetilde{\Xi}\left(\mathbf{p}^{\prime \prime}, \mathbf{p}+\mathbf{p}^{\prime}-\mathbf{p}^{\prime \prime}\right) .
$$

The free action can therefore be written as follows:

$$
A=\int d t \frac{d \mathbf{p}}{(2 \pi)^{3}} \frac{d \mathbf{p}^{\prime}}{(2 \pi)^{3}} \chi^{\dagger}(\mathbf{p}) S^{-1}\left(\mathbf{p}, \mathbf{p}^{\prime}\right) \chi\left(\mathbf{p}^{\prime}\right),
$$

with

$$
S^{-1}\left(\mathbf{p}, \mathbf{p}^{\prime}\right)=(2 \pi)^{3}\left(\begin{array}{cc}
\left(i \partial_{t}-\xi_{\mathbf{p}}+\delta \mu \sigma_{3}\right) \delta\left(\mathbf{p}-\mathbf{p}^{\prime}\right) & -\Delta\left(\mathbf{p}, \mathbf{p}^{\prime}\right) \\
-\Delta^{*}\left(\mathbf{p}, \mathbf{p}^{\prime}\right) & \left(i \partial_{t}+\xi_{\mathbf{p}}+\delta \mu \sigma_{3}\right) \delta\left(\mathbf{p}-\mathbf{p}^{\prime}\right)
\end{array}\right)
$$

Here

$$
\xi_{\mathbf{p}}=E(\mathbf{p})-\mu \approx \mathbf{v}_{F} \cdot\left(\mathbf{p}-\mathbf{p}_{F}\right),
$$

where

$$
\mathbf{v}_{F}=\left.\frac{\partial E(\mathbf{p})}{\partial \mathbf{p}}\right|_{\mathbf{p}=\mathbf{p}_{F}}
$$

is the Fermi velocity. We have used the fact that we are considering only degrees of freedom near the Fermi surface, i.e.,

$$
p_{F}-\delta<p<p_{F}+\delta,
$$

where $\delta$ is the ultraviolet cutoff, of the order of the Debye frequency. In particular in the nonrelativistic case

$$
\xi_{\mathbf{p}}=\frac{\mathbf{p}^{2}}{2 m}-\frac{p_{F}^{2}}{2 m} \text { and } \mathbf{v}_{F}=\frac{\mathbf{p}_{F}}{m} .
$$

$S^{-1}$ in Eq. (2.15) is the 3D Fourier transform of the inverse propagator. We can make the energy dependence explicit by Fourier-transforming the time variable as well. In this way, we get for the inverse propagator written as an operator

$$
S^{-1}=\left(\begin{array}{cc}
\left(\mathbf{G}_{\mathbf{0}}^{+}\right)^{-1} & -\boldsymbol{\Delta} \\
-\boldsymbol{\Delta}^{*} & -\left(\mathbf{G}_{\mathbf{0}}^{-}\right)^{-\mathbf{1}}
\end{array}\right),
$$

and

$$
\begin{aligned}
& {\left[\mathbf{G}_{\mathbf{0}}^{+}\right]^{-\mathbf{1}}=E-\xi_{\mathbf{P}}+\delta \mu \sigma_{3}+i \epsilon \operatorname{sgn} E,} \\
& {\left[\mathbf{G}_{\mathbf{0}}^{-}\right]^{-\mathbf{1}}=-E-\xi_{\mathbf{P}}-\delta \mu \sigma_{3}-i \epsilon \operatorname{sgn} E,}
\end{aligned}
$$

with $\epsilon=0^{+}$and $\mathbf{P}$ the momentum operator. The $i \epsilon$ prescription is nothing but the usual one for the Feynman propagator, that is, forward propagation in time for the positive-energy solutions and backward propagation for the negative-energy solutions. As for the NambuGor'kov propagator $S$, one gets

$$
S=\left(\begin{array}{cc}
\mathbf{G} & -\widetilde{\mathbf{F}} \\
-\mathbf{F} & \widetilde{\mathbf{G}}
\end{array}\right) .
$$

$S$ has both spin, $\sigma, \sigma^{\prime}$, and $a, b$ indices, i.e., $S_{\sigma \sigma^{\prime}}^{a b} .^{2}$ The Nambu-Gor'kov equations in compact form are

$$
S^{-1} S=1,
$$

or, explicitly,

$$
\begin{aligned}
& {\left[\mathbf{G}_{\mathbf{0}}^{+}\right]^{-1} \mathbf{G}+\boldsymbol{\Delta} \mathbf{F}=\mathbf{1},} \\
& -\left[\mathbf{G}_{\mathbf{0}}^{-}\right]^{-1} \mathbf{F}+\boldsymbol{\Delta}^{*} \mathbf{G}=\mathbf{0} .
\end{aligned}
$$

Note that we shall use

$$
\left\langle\mathbf{r}|\Delta| \mathbf{r}^{\prime}\right\rangle=\frac{g}{2} \Xi(\mathbf{r}) \delta\left(\mathbf{r}-\mathbf{r}^{\prime}\right)=\Delta(\mathbf{r}) \delta\left(\mathbf{r}-\mathbf{r}^{\prime}\right)
$$

or

$$
\left\langle\mathbf{p}|\Delta| \mathbf{p}^{\prime}\right\rangle=\Delta\left(\mathbf{p}, \mathbf{p}^{\prime}\right),
$$

depending on our choice of the coordinate or momentum representation. The formal solution of the system (2.24) is

$$
\begin{aligned}
& \mathbf{F}=\mathbf{G}_{\mathbf{0}}^{-} \Delta^{*} \mathbf{G}, \\
& \mathbf{G}=\mathbf{G}_{\mathbf{0}}^{+}-\mathbf{G}_{\mathbf{0}}^{+} \Delta \mathbf{F},
\end{aligned}
$$

so that $\mathbf{F}$ satisfies the equation

$$
\mathbf{F}=\mathbf{G}_{0}^{-} \Delta^{*}\left(\mathbf{G}_{0}^{+}-\mathbf{G}_{0}^{+} \Delta \mathbf{F}\right)
$$

and is therefore given by

$$
\mathbf{F}=\frac{1}{\boldsymbol{\Delta}^{*}\left[\mathbf{G}_{0}^{+}\right]^{-\mathbf{1}}\left[\boldsymbol{\Delta}^{*}\right]^{-1}\left[\mathbf{G}_{\mathbf{0}}^{-}\right]^{-\mathbf{1}}+\boldsymbol{\Delta}^{*} \boldsymbol{\Delta}} \boldsymbol{\Delta}^{*} .
$$

In the configuration space, the Nambu-Gor'kov equations (2.24) are as follows:

\footnotetext{
${ }^{2}$ We note that the presence of the factor $1 / \sqrt{2}$ in Eq. (2.11) implies an extra factor of 2 in the propagator: $S\left(x, x^{\prime}\right)$ $=2\left\langle T\left\{\chi(x) \chi^{\dagger}\left(x^{\prime}\right)\right\}\right\rangle$, as can be seen considering, e.g., the matrix element $S^{11}:\left\langle T\left\{\psi(x) \psi^{\dagger}\left(x^{\prime}\right)\right\}\right\rangle=\left(i \partial_{t}-\xi_{-i \vec{\nabla}}-\delta \mu \sigma_{3}\right)^{-1} \delta(x$ $\left.-x^{\prime}\right)$, with $(x \equiv(t, \mathbf{r}))$.
} 


$$
\begin{aligned}
& {\left[E-E(-i \nabla)+\mu+\delta \mu \sigma_{3}\right] G\left(\mathbf{r}, \mathbf{r}^{\prime}, E\right)+\Delta(\mathbf{r}) F\left(\mathbf{r}, \mathbf{r}^{\prime}, E\right)} \\
& \quad=\delta\left(\mathbf{r}-\mathbf{r}^{\prime}\right), \\
& {\left[-E-E(-i \nabla)+\mu-\delta \mu \sigma_{3}\right] F\left(\mathbf{r}, \mathbf{r}^{\prime}, E\right)} \\
& \quad-\Delta^{*}(\mathbf{r}) G\left(\mathbf{r}, \mathbf{r}^{\prime}, E\right)=0 .
\end{aligned}
$$

The gap equation at $T=0$ is the following consistency condition:

$$
\Delta^{*}(\mathbf{r})=-i \frac{g}{2} \int \frac{d E}{2 \pi} \operatorname{Tr} F(\mathbf{r}, \mathbf{r}, E),
$$

where $F$ is given by Eq. (2.29). To derive the gap equation we observe that

$$
\begin{aligned}
\Delta^{*}(\mathbf{r})= & \frac{g}{2} \Xi^{*}(\mathbf{r}) \\
= & \frac{g}{2} \int \frac{d \mathbf{p}_{1}}{(2 \pi)^{3}} \frac{d \mathbf{p}_{2}}{(2 \pi)^{3}} e^{i\left(\mathbf{p}_{1}+\mathbf{p}_{2}\right) \cdot \mathbf{r} \Xi *\left(\mathbf{p}_{1}, \mathbf{p}_{2}\right)} \\
= & -\frac{g}{2} \int \frac{d E}{2 \pi} \frac{d \mathbf{p}_{1}}{(2 \pi)^{3}} \frac{d \mathbf{p}_{2}}{(2 \pi)^{3}} e^{i\left(\mathbf{p}_{1}+\mathbf{p}_{2}\right) \cdot \mathbf{r}} \\
& \times\left\langle\psi^{\dagger}\left(\mathbf{p}_{1}, E\right) \psi^{c}\left(\mathbf{p}_{2}, E\right)\right\rangle \\
= & +i \frac{g}{2} \sum_{\sigma} \int \frac{d E}{2 \pi} \frac{d \mathbf{p}_{1}}{(2 \pi)^{3}} \frac{d \mathbf{p}_{2}}{(2 \pi)^{3}} \\
& \times e^{i\left(\mathbf{p}_{1}-\mathbf{p}_{2}\right) \cdot \mathbf{r}} S_{\sigma \sigma}^{21}\left(\mathbf{p}_{2}, \mathbf{p}_{1}\right) \\
= & +i \frac{g}{2} \sum_{\sigma} \int \frac{d E}{2 \pi} S_{\sigma \sigma}^{21}(\mathbf{r}, \mathbf{r}),
\end{aligned}
$$

which gives Eq. (2.31).

At finite temperature, and introducing the Matsubara frequencies $\omega_{n}=(2 n+1) \pi T$, the gap equation reads

$$
\Delta^{*}(\mathbf{r})=\left.\frac{g}{2} T \sum_{n=-\infty}^{+\infty} \operatorname{Tr} F(\mathbf{r}, \mathbf{r}, E)\right|_{E=i \omega_{n}} .
$$

\section{B. Homogeneous superconductors and their phase diagram}

It is useful to specialize these relations to the case of homogeneous materials. In this case we have

$$
\begin{aligned}
& \Xi(\mathbf{r})=\mathrm{const} \equiv \frac{2 \Delta}{g}, \\
& \widetilde{\Xi}\left(\mathbf{p}_{1}, \mathbf{p}_{2}\right)=\frac{2 \Delta}{g} \frac{\pi^{2}}{p_{F}^{2} \delta}(2 \pi)^{3} \delta\left(\mathbf{p}_{1}+\mathbf{p}_{2}\right) .
\end{aligned}
$$

Therefore we get

$$
\Delta\left(\mathbf{p}_{1}, \mathbf{p}_{2}\right)=\Delta \delta\left(\mathbf{p}_{1}-\mathbf{p}_{2}\right)
$$

and, from Eqs. (2.25) and (2.34),

$$
\Delta(\mathbf{r})=\Delta *(\mathbf{r})=\Delta .
$$

Therefore $F(\mathbf{r}, \mathbf{r}, E)$ is independent of $\mathbf{r}$ and, from Eq. (2.29), we get

$$
\operatorname{Tr} F(\mathbf{r}, \mathbf{r}, E)=-2 \Delta \int \frac{d^{3} p}{(2 \pi)^{3}} \frac{1}{(E-\delta \mu)^{2}-\xi_{\mathbf{p}}^{2}-\Delta^{2}},
$$

which gives the gap equation at $T=0$,

$$
\Delta=i g \Delta \int \frac{d E}{2 \pi} \frac{d^{3} p}{(2 \pi)^{3}} \frac{1}{(E-\delta \mu)^{2}-\xi_{\mathbf{p}}^{2}-\Delta^{2}},
$$

and at $T \neq 0$,

$$
\Delta=g T \sum_{n=-\infty}^{+\infty} \int \frac{d^{3} p}{(2 \pi)^{3}} \frac{\Delta}{\left(\omega_{n}+i \delta \mu\right)^{2}+\epsilon(\mathbf{p}, \Delta)^{2}},
$$

with

$$
\epsilon(\mathbf{p}, \Delta)=\sqrt{\Delta^{2}+\xi_{\mathbf{p}}^{2}}
$$

We now use the identity

$$
\begin{aligned}
\frac{1}{2}\left[1-n_{u}-n_{d}\right] & \\
& =\epsilon(\mathbf{p}, \Delta) T \sum_{n=-\infty}^{+\infty} \frac{1}{\left(\omega_{n}+i \delta \mu\right)^{2}+\epsilon^{2}(\mathbf{p}, \Delta)},
\end{aligned}
$$

where

$$
n_{u}(\mathbf{p})=\frac{1}{e^{(\epsilon+\delta \mu) / T}+1} \text { and } n_{d}(\mathbf{p})=\frac{1}{e^{(\epsilon-\delta \mu) / T}+1} .
$$

The gap equation can be therefore written as

$$
\Delta=\frac{g \Delta}{2} \int \frac{d^{3} p}{(2 \pi)^{3}} \frac{1}{\epsilon(\mathbf{p}, \Delta)}\left[1-n_{u}(\mathbf{p})-n_{d}(\mathbf{p})\right] .
$$

In the Landau theory of the Fermi liquid, $n_{u}$ and $n_{d}$ are interpreted as the equilibrium distributions for quasiparticles of types $u$ and $d$. It should be noted that the last two terms act as blocking factors, reducing the phase space and eventually producing $\Delta \rightarrow 0$ when $T$ reaches a critical value $T_{c}$ (see below).

Before considering the solutions of the gap equations in the general case let us first consider the case $\delta \mu=0$; the corresponding gap is denoted $\Delta_{0}$. At $T=0$ there is no reduction of the phase space, and the gap equation becomes

$$
1=\frac{g}{2} \int \frac{d^{3} p}{(2 \pi)^{3}} \frac{1}{\epsilon\left(\mathbf{p}, \Delta_{0}\right)},
$$

whose solution (assuming $d^{3} p=p_{F}^{2} d p d \Omega$ ) is

$$
\Delta_{0}=\frac{\delta}{\sinh \frac{2}{g \rho}} .
$$

Here

$$
\rho=\frac{p_{F}^{2}}{\pi^{2} v_{F}}
$$

is the density of states and we have used $\xi_{\mathbf{p}} \approx v_{F}(p$ $-p_{F}$ ) [see Eqs. (2.16)-(2.19)]. In the weak-coupling limit Eq. (2.46) gives 


$$
\Delta_{0}=2 \delta e^{-2 / \rho g} .
$$

Let us now consider the case $\delta \mu \neq 0$. By Eq. (2.44) the gap equation is written as

$$
-1+\frac{g}{2} \int \frac{d^{3} p}{(2 \pi)^{3}} \frac{1}{\epsilon}=\frac{g}{2} \int \frac{d^{3} p}{(2 \pi)^{3}} \frac{n_{u}+n_{d}}{\epsilon} .
$$

Using the gap equation for the BCS superconductor, we can write the left-hand side (lhs), in the weak-coupling limit, as

$$
\mathrm{lhs}=\frac{g \rho}{2} \ln \frac{\Delta_{0}}{\Delta},
$$

where we got rid of the cutoff $\delta$ by using $\Delta_{0}$, the gap at $\delta \mu=0$, and $T=0$. Let us now evaluate the right-hand side (rhs) at $T=0$. We get

$$
\left.\operatorname{rhs}\right|_{T=0}=\frac{g \rho}{2} \int_{0}^{\delta} \frac{d \xi_{\mathbf{p}}}{\epsilon}[\theta(-\epsilon-\delta \mu)+\theta(-\epsilon+\delta \mu)] .
$$

The gap equation at $T=0$ can therefore be written as follows:

$$
\ln \frac{\Delta_{0}}{\Delta}=\theta(\delta \mu-\Delta) \operatorname{arcsinh} \frac{\sqrt{\delta \mu^{2}-\Delta^{2}}}{\Delta}
$$

i.e.,

$$
\ln \frac{\Delta_{0}}{\delta \mu+\sqrt{\delta \mu^{2}-\Delta^{2}}}=0
$$

One can immediately see that there are no solutions for $\delta \mu>\Delta_{0}$. For $\delta \mu \leqslant \Delta_{0}$ one has two solutions:
(a) $\Delta=\Delta_{0}$
(b) $\Delta^{2}=2 \delta \mu \Delta_{0}-\Delta_{0}^{2}$.

The first arises since for $\Delta=\Delta_{0}$, the lhs of Eq. (2.52) is zero. But since we may have solutions only for $\delta \mu$ $\leqslant \Delta_{0}$, the $\theta$ function in Eq. (2.52) also makes the rhs zero. The existence of this solution can also be seen from Eq. (2.39). In fact, in this equation one can shift the integration variable $E \rightarrow E+\delta \mu$, getting the result that, in the superconductive phase, the gap $\Delta$ is independent of $\delta \mu$, i.e., $\Delta=\Delta_{0}$.

To compute the free energy we make use of the theorem saying that, for small variations of an external parameter of the system, all the thermodynamical quantities vary in the same way (Landau and Lifshitz, 1996). We apply this to the grand potential to get

$$
\frac{\partial \Omega}{\partial g}=\left\langle\frac{\partial H}{\partial g}\right\rangle .
$$

From the expression for the interaction Hamiltonian [see Eq. (2.5)], we immediately get (cf. Abrikosov et al., 1963, Chap. 7)

$$
\Omega=-\int \frac{d g}{g^{2}} \int d \mathbf{x}|\Delta(\mathbf{x})|^{2} .
$$

For homogeneous media this gives

$$
\frac{\Omega}{V}=-\int \frac{d g}{g^{2}}|\Delta|^{2} .
$$

Using the result (2.48), we can trade the integration over the coupling constant $g$ for an integration over $\Delta_{0}$, the BCS gap at $\delta \mu=0$, because $d \Delta_{0} / \Delta_{0}=2 d g / \rho g^{2}$. Therefore the difference in free energy between the superconductor and the normal state is (we shall use interchangeably the symbol $\Omega$ for the grand potential and its density $\Omega / V)$

$$
\Omega_{\Delta}-\Omega_{0}=-\frac{\rho}{2} \int_{\Delta_{f}}^{\Delta_{0}} \Delta^{2} \frac{d \Delta_{0}}{\Delta_{0}} .
$$

Here $\Delta_{f}$ is the value of $\Delta_{0}$ corresponding to $\Delta=0 . \Delta_{f}$ $=0$ in case (a), Eq. (2.54), and $\Delta_{f}=2 \delta \mu$ in case (b) Eq. (2.55); in the latter case we see immediately that $\Omega_{\Delta}$ $-\Omega_{0}>0$ because from Eq. (2.55) it follows that $\Delta_{0}$ $<2 \delta \mu$. The free energies for $\delta \mu \neq 0$ corresponding to the cases (a) and (b) above can be computed by substituting Eq. (2.54) and Eq. (2.55) into Eq. (2.59). Before doing that, let us derive the density of free energy at $T$ $=0$ and $\delta \mu \neq 0$ in the normal nonsuperconducting state. Let us start from the very definition of the grand potential for free spin- $1 / 2$ particles:

$$
\Omega_{0}(0, T)=-2 V T \int \frac{d^{3} p}{(2 \pi)^{3}} \ln \left(1+e^{[\mu-\epsilon(\mathbf{p})] / T}\right) .
$$

Integrating this expression by parts we get, for $T \rightarrow 0$,

$$
\Omega_{0}(0)=-\frac{V}{12 \pi^{3}} \int d \Omega_{\mathbf{p}} p^{3} d \epsilon \theta(\mu-\epsilon) .
$$

From this expression we can easily evaluate the grand potential for two fermions with different chemical potentials, expanding at the first nontrivial order in $\delta \mu / \mu$. The result is

$$
\Omega_{0}(\delta \mu)=\Omega_{0}(0)-\frac{\delta \mu^{2}}{2} \rho .
$$

Therefore from Eqs. (2.54), (2.55), and (2.59) in cases (a) and (b) we have

$$
\text { (a) } \Omega_{\Delta}(\delta \mu)=\Omega_{0}(\delta \mu)-\frac{\rho}{4}\left(-2 \delta \mu^{2}+\Delta_{0}^{2}\right)
$$

and

$$
\text { (b) } \Omega_{\Delta}(\delta \mu)=\Omega_{0}(\delta \mu)-\frac{\rho}{4}\left(-4 \delta \mu^{2}+4 \delta \mu \Delta_{0}-\Delta_{0}^{2}\right) \text {. }
$$

Comparing Eqs. (2.63) and (2.64) we see that the solution (a) has lower $\Omega$. Therefore, for $\delta \mu<\Delta_{0} / \sqrt{2}$, the BCS superconductive state is stable (Clogston, 1962). At $\delta \mu=\Delta_{0} / \sqrt{2}$ it becomes metastable, as the normal state has a lower free energy. This transition would be first order, since the gap does not depend on $\delta \mu$.

The grand potentials for the two cases (a) and (b) and for the gapless phase, Eq. (2.62), are depicted in Fig. 1, together with the corresponding gaps.

A different proof is obtained by integrating the gap equation written in the form 


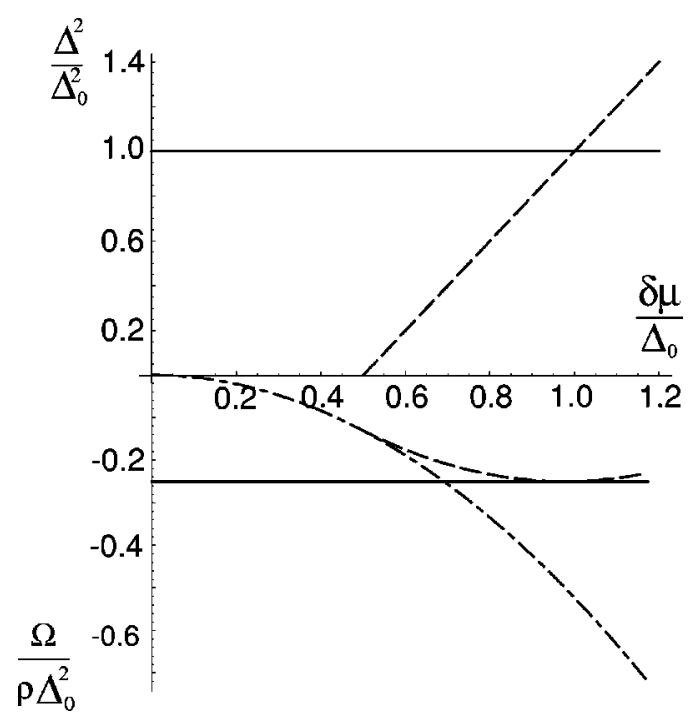

FIG. 1. Gap and grand potential as functions of $\delta \mu$ for the two solutions (a) and (b) discussed in the text; see Eqs. (2.54), (2.55), (2.63), and (2.64). Upper solid line, gap for solution (a); upper dashed line, gap for solution (b). In the lower part we plot the grand potential: solid line, for solution (a); dashed line, for solution (b); dashed-dotted line for the normal gapless state with $\delta \mu \neq 0$. All the grand potentials are referred to the value $\Omega_{0}(0)$ (normal state with $\delta \mu=0$ )

$$
\frac{\partial \Omega}{\partial \Delta}=0 .
$$

Normalization can be obtained by considering the homogeneous case with $\delta \mu=0$, when, in the weak-coupling limit, from Eqs. (2.57) and (2.48) one gets

$$
\Omega=-\frac{\rho}{4} \Delta_{0}^{2}
$$

[see Eq. (2.71) below]. In this way one obtains again the results of Eqs. (2.63) and (2.64).

This analysis shows that at $\delta \mu=\delta \mu_{1}=\Delta_{0} / \sqrt{2}$ one would pass abruptly from the superconducting $(\Delta \neq 0)$ to the normal $(\Delta=0)$ phase. However, as we shall discuss below, the real ground state for $\delta \mu>\delta \mu_{1}$ turns out to be an inhomogeneous one, where the assumption in Eq. (2.37) of a uniform gap is not justified.

Let us now consider the phase diagram of a homogeneous superconductor for small values of the gap parameter, which allows us to perform a Ginzburg-Landau expansion of the gap equation and grand potential. In order to perform a complete study, we need to expand the grand potential up to sixth order in the gap. As a matter of fact, in the plane $(\delta \mu, T)$ there is a first-order transition at $\left(\delta \mu_{1}, 0\right)$, and a second-order one at $\left(0, T_{c}\right)$ (the usual BCS second-order transition). Therefore we expect that second-order and first-order lines start from these points and meet at a tricritical point, which by definition is the meeting point of a second-order and a first-order transition line. A tricritical point is characterized by the simultaneous vanishing of the $\Delta^{2}$ and $\Delta^{4}$ coefficients in the grand-potential expansion, which is why we need to introduce the sixth-order term in the grand potential. For stability reasons the corresponding coefficient should be positive; if not, we should also include the $\Delta^{8}$ term.

We consider the grand potential, as measured from the normal state, near a second-order phase transition:

$$
\Omega=\frac{1}{2} \alpha \Delta^{2}+\frac{1}{4} \beta \Delta^{4}+\frac{1}{6} \gamma \Delta^{6} .
$$

Minimization gives the gap equation:

$$
\alpha \Delta+\beta \Delta^{3}+\gamma \Delta^{5}=0 .
$$

Expanding Eq. (2.40) up to fifth order in $\Delta$ and comparing with the previous equation, we determine the coefficients $\alpha, \beta$, and $\gamma$ up to a normalization constant. We get

$$
\begin{aligned}
\Delta= & 2 g \rho T \operatorname{Re} \sum_{n=0}^{\infty} \int_{0}^{\delta} d \xi\left[\frac{\Delta}{\left(\bar{\omega}_{n}^{2}+\xi^{2}\right)}-\frac{\Delta^{3}}{\left(\bar{\omega}_{n}^{2}+\xi^{2}\right)^{2}}\right. \\
& \left.+\frac{\Delta^{5}}{\left(\bar{\omega}_{n}^{2}+\xi^{2}\right)^{3}}+\cdots\right]
\end{aligned}
$$

with

$$
\bar{\omega}_{n}=\omega_{n}+i \delta \mu=(2 n+1) \pi T+i \delta \mu .
$$

The grand potential can be obtained, up to a normalization factor, by integrating the gap equation over $\Delta$. The normalization can be obtained by the simple BCS case, considering the grand potential as obtained, in the weakcoupling limit, from Eqs. (2.57) and (2.48):

$$
\Omega=-\frac{\rho}{4} \Delta_{0}^{2} .
$$

The same result can be obtained by multiplying the gap equation (2.45) by $\Delta_{0}$ and integrating the result, provided that we multiply it by the factor $2 / g$, which fixes the normalization. Therefore

$$
\begin{aligned}
& \alpha=\frac{2}{g}\left(1-2 g \rho T \operatorname{Re} \sum_{n=0}^{\infty} \int_{0}^{\delta} \frac{d \xi}{\left(\bar{\omega}_{n}^{2}+\xi^{2}\right)}\right), \\
& \beta=4 \rho T \operatorname{Re} \sum_{n=0}^{\infty} \int_{0}^{\infty} \frac{d \xi}{\left(\bar{\omega}_{n}^{2}+\xi^{2}\right)^{2}}, \\
& \gamma=-4 \rho T \operatorname{Re} \sum_{n=0}^{\infty} \int_{0}^{\infty} \frac{d \xi}{\left(\bar{\omega}_{n}^{2}+\xi^{2}\right)^{3}} .
\end{aligned}
$$

In the coefficients $\beta$ and $\gamma$ we have extended the integration in $\xi$ up to infinity since both the sum and the integral are convergent. To evaluate $\alpha$ is less trivial. We can proceed in two different ways. We can sum over the Matsubara frequencies and then integrate over $\xi$, or we can perform the operations in the inverse order. Let us begin with the former method. We get

$$
\alpha=\frac{2}{g}\left\{1-\frac{g \rho}{4} \int_{0}^{\delta} \frac{d \xi}{\xi}\left[\tanh \left(\frac{\xi-\mu}{2 T}\right)+\tanh \left(\frac{\xi+\mu}{2 T}\right)\right]\right\} .
$$

Performing an integration by parts, we can extract the logarithmic divergence in $\delta$. This can be eliminated using the result (2.46), valid for $\delta \mu=T=0$ in the weakcoupling limit, 


$$
1=\frac{g \rho}{2} \ln \frac{2 \delta}{\Delta_{0}} .
$$

We find

$$
\begin{aligned}
\alpha=\rho\left[\ln \frac{2 T}{\Delta_{0}}+\frac{1}{4} \int_{0}^{\infty} d x \ln x\left(\frac{1}{\cosh ^{2} \frac{x+y}{2}}\right.\right. \\
\left.\left.+\frac{1}{\cosh ^{2} \frac{x-y}{2}}\right)\right],
\end{aligned}
$$

where

$$
y=\frac{\delta \mu}{T} .
$$

Defining

$\ln \frac{\Delta_{0}}{2 T_{c}(y)}=\frac{1}{4} \int_{0}^{\infty} d x \ln x\left(\frac{1}{\cosh ^{2} \frac{x+y}{2}}+\frac{1}{\cosh ^{2} \frac{x-y}{2}}\right)$,

we get

$$
\alpha(v, t)=\rho \ln \frac{t}{t_{c}(v / t)},
$$

where

$$
v=\frac{\delta \mu}{\Delta_{0}}, \quad t=\frac{T}{\Delta_{0}}, \quad t_{c}=\frac{T_{c}}{\Delta_{0}} .
$$

Therefore the equation

$$
t=t_{c}(v / t)
$$

defines the line of the second-order phase transition. Performing the calculation in the reverse order brings us to a more manageable result for $t_{c}(y)$ (Buzdin and Kachkachi, 1997). In Eq. (2.72), we first integrate over $\xi$, obtaining a divergent series which can be regulated by cutting the sum at a maximal value of $n$ determined by

$$
\omega_{N}=\delta \Rightarrow N \approx \frac{\delta}{2 \pi T} .
$$

We obtain

$$
\alpha=\frac{2}{g}\left(1-\pi g \rho T \operatorname{Re} \sum_{n=0}^{N} \frac{1}{\bar{\omega}_{n}}\right) .
$$

The sum can be performed in terms of the Euler function $\psi(z)$ :

$$
\begin{aligned}
\operatorname{Re} \sum_{n=0}^{N} \frac{1}{\bar{\omega}_{n}}= & \frac{1}{2 \pi T} \operatorname{Re}\left[\psi\left(\frac{3}{2}+i \frac{y}{2 \pi}+N\right)\right. \\
& \left.-\psi\left(\frac{1}{2}+i \frac{y}{2 \pi}\right)\right] \\
\approx & \frac{1}{2 \pi T}\left[\ln \frac{\delta}{2 \pi T}-\operatorname{Re} \psi\left(\frac{1}{2}+i \frac{y}{2 \pi}\right)\right] .
\end{aligned}
$$

Eliminating the cutoff as we did before, we get

$$
\alpha(v, t)=\rho\left[\ln (4 \pi t)+\operatorname{Re} \psi\left(\frac{1}{2}+i \frac{v}{2 \pi t}\right)\right] .
$$

By comparing this with Eq. (2.77) we get the following identity:

$$
\begin{aligned}
\operatorname{Re} \psi\left(\frac{1}{2}+i \frac{y}{2 \pi}\right)= & -\ln (2 \pi)+\frac{1}{4} \int_{0}^{\infty} d x \ln x\left(\frac{1}{\cosh ^{2} \frac{x+y}{2}}\right. \\
& \left.+\frac{1}{\cosh ^{2} \frac{x-y}{2}}\right)
\end{aligned}
$$

Equation (2.79) can be rewritten as

$$
\ln \frac{\Delta_{0}}{4 \pi T_{c}(y)}=\operatorname{Re} \psi\left(\frac{1}{2}+i \frac{y}{2 \pi}\right) .
$$

In particular, at $\delta \mu=0$, using

$$
\psi\left(\frac{1}{2}\right)=-\ln (4 \gamma), \quad \gamma=e^{C}, \quad C=0.5777 \ldots,
$$

where $C$ is the Euler-Mascheroni constant, we find from Eq. (2.86)

$$
\alpha\left(0, T / \Delta_{0}\right)=\rho \ln \frac{\pi T}{\gamma \Delta_{0}}
$$

reproducing the critical temperature for the BCS case:

$$
T_{c}=\frac{\gamma}{\pi} \Delta_{0} \approx 0.56693 \Delta_{0}
$$

The other terms in the expansion of the gap equation are easily evaluated by integrating over $\xi$ and summing over the Matsubara frequencies. We get

$$
\begin{aligned}
\beta & =\pi \rho T \operatorname{Re} \sum_{n=0}^{\infty} \frac{1}{\bar{\omega}_{n}^{3}} \\
& =-\frac{\rho}{16 \pi^{2} T^{2}} \operatorname{Re} \psi^{(2)}\left(\frac{1}{2}+i \frac{\delta \mu}{2 \pi T}\right), \\
\gamma & =-\frac{3}{4} \pi \rho T \operatorname{Re} \sum_{n=0}^{\infty} \frac{1}{\bar{\omega}_{n}^{5}} \\
& =\frac{3}{4} \frac{\rho}{768 \pi^{4} T^{4}} \operatorname{Re} \psi^{(4)}\left(\frac{1}{2}+i \frac{\delta \mu}{2 \pi T}\right),
\end{aligned}
$$

where

$$
\psi^{(n)}(z)=\frac{d^{n}}{d z^{n}} \psi(z)
$$

Let us now briefly review some results on the grand potential in the Ginzburg-Landau expansion (2.67). We shall assume $\gamma>0$ in order to ensure the stability of the potential. Minimization leads to the solutions

$$
\begin{aligned}
& \Delta=0 \\
& \Delta^{2}=\Delta_{ \pm}^{2}=\frac{1}{2 \gamma}\left(-\beta \pm \sqrt{\beta^{2}-4 \alpha \gamma}\right) .
\end{aligned}
$$




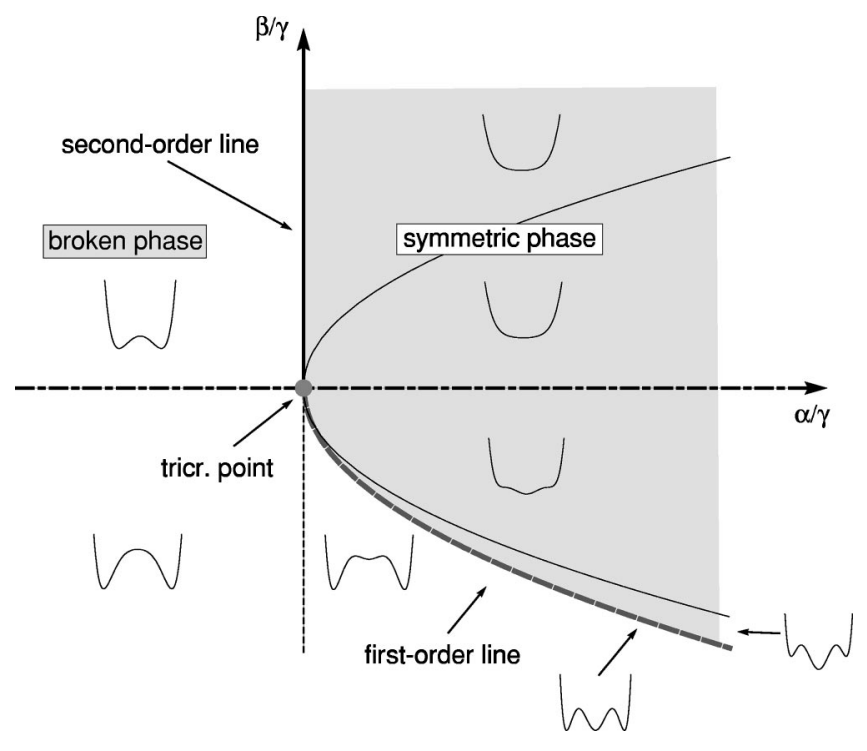

FIG. 2. First-order and second-order transition lines for the potential of Eq. (2.67). We show the tricritical point and the regions corresponding to the symmetric and the broken phases. Also shown is the behavior of the grand potential in the various regions. The thin solid line is the locus of the points $\beta^{2}-4 \alpha \gamma=0$. In the interior region we have $\beta^{2}-4 \alpha \gamma<0$.

The discussion of the minima of $\Omega$ depends on the signs of the parameters $\alpha$ and $\beta$. The results are the following:

(1) $\alpha>0, \beta>0$. In this case there is a single minimum given by Eq. (2.95) and the phase is symmetric.

(2) $\alpha>0, \beta<0$. Here there are three minima, one given by Eq. (2.95) and the other two degenerate minima at

$$
\Delta= \pm \Delta_{+} .
$$

The line along which the three minima become equal is given by

$$
\Omega(0)=\Omega\left( \pm \Delta_{+}\right) \rightarrow \beta=-4 \sqrt{\frac{\alpha \gamma}{3}} .
$$

Along this line there is a first-order transition with a discontinuity in the gap given by

$$
\Delta_{+}^{2}=-\frac{4 \alpha}{\beta}=-\frac{3}{4} \frac{\beta}{\gamma} .
$$

To the right of the first-order line we have $\Omega(0)$ $<\Omega\left( \pm \Delta_{+}\right)$. It follows that to the right of this line there is the symmetric phase, whereas to the left is the broken phase (see Fig. 2).

(3) $\alpha<0, \beta>0$. In this case Eq. (2.95) gives a maximum, and there are two degenerate minima given by Eq. (2.97). Since for $\alpha>0$ the two minima disappear, it follows that there is a second-order phase transition along the line $\alpha=0$. This can also be seen by noticing that going from the broken phase to the symmetric one we have

$$
\lim _{\alpha \rightarrow 0} \Delta_{+}^{2}=0
$$

(4) $\alpha<0, \beta<0$. The minima and the maximum are as in the previous case.

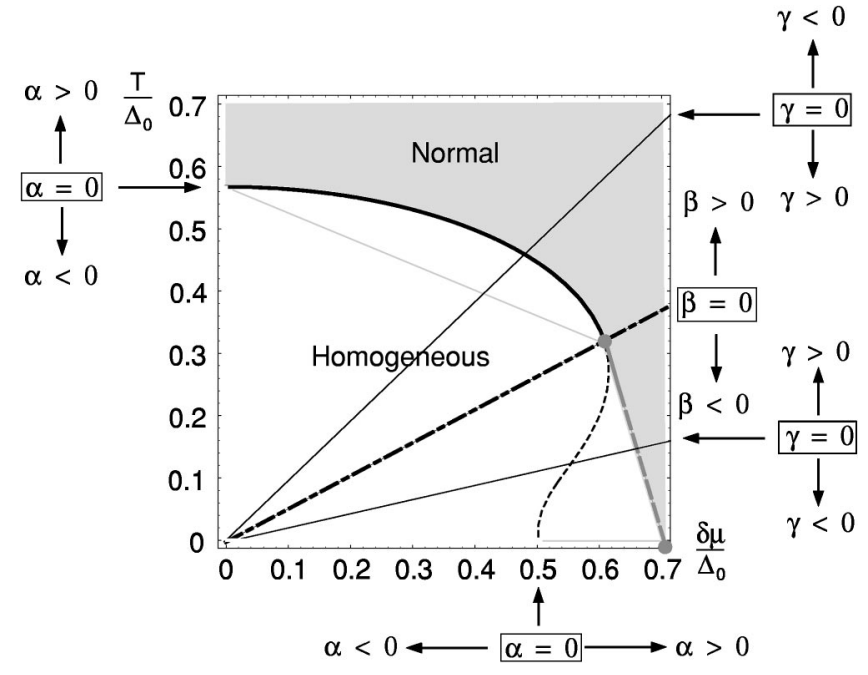

FIG. 3. The point solutions of the equation $\Delta=0$ in the plane $(v, t)=\left(\delta \mu / \Delta_{0}, T / \Delta_{0}\right)$. The tricritical point at $(\delta \mu, T)$ $\approx(0.61,0.32) \Delta_{0}$ is also shown. The upper part of the curve (solid line) separates the homogeneous phase from the normal one. Along the dashed line $\Delta=0$, but this is not the absolute minimum of the grand potential.

Notice also that the solutions $\Delta_{ \pm}$do not exist in the region $\beta^{2}<4 \alpha \gamma$. This situation is summarized in Fig. 2, where we show the behavior of the grand potential in the different sectors of the plane $(\alpha / \gamma, \beta / \gamma)$, together with the transition lines. In the quadrant $(\alpha>0, \beta<0)$ there are metastable phases corresponding to nonabsolute minima. In the sector included between the line $\beta$ $=-2 \sqrt{\alpha / \gamma}$ and the first-order transition line the metastable phase is the broken one, whereas in the region between the first-order and the $\alpha=0$ lines the metastable phase is the symmetric one.

Using Eqs. (2.86), (2.92), and (2.93), which give the parameters $\alpha, \beta$, and $\gamma$ in terms of the variables $v$ $=\delta \mu / \Delta_{0}$ and $t=T / \Delta_{0}$, we can map the plane $\alpha$ and $\beta$ into the plane $\left(\delta \mu / \Delta_{0}, T / \Delta_{0}\right)$. The result is shown in Fig. 3. From this mapping we can draw several conclusions. First, the region where the previous discussion applies in terms of the parameters $\alpha, \beta$, and $\gamma$ is inside the triangular part delimited by the lines $\gamma=0$. In fact, as already stressed, our expansion does not hold outside this region. This statement can be made quantitative by noticing that along the first-order transition line the gap increases when going away from the tricritical point as

$$
\Delta_{+}^{2}=-\frac{4 \alpha}{\beta}=\sqrt{\frac{3 \alpha}{\gamma}} .
$$

Notice that the lines $\beta(v, t)=0$ and $\gamma(v, t)=0$ are straight lines, since these zeros are determined by the functions $\psi^{(2)}$ and $\psi^{(4)}$, which depend only on the ratio $v / t$. Calculating the first-order line around the tricritical point one gets the result plotted as a solid line in Fig. 3. Since we know that $\delta \mu=\delta \mu_{1}=\Delta_{0} / \sqrt{2}$ is a first-order 
transition point, the first-order line must end there. In Fig. 3 we have simply connected the line and the point with a gray dashed line. To obtain this line, a numerical evaluation at all orders in $\Delta$ would be required. This is feasible, but we shall skip it since the results will not be necessary in the following (see Sarma, 1963). The location of the tricritical point is determined by the intersection of the lines $\alpha=0$ and $\beta=0$. One finds (Buzdin and Kachkachi, 1997; Combescot and Mora, 2002)

$$
\left.\frac{\delta \mu}{\Delta_{0}}\right|_{\text {tric }}=0.60822,\left.\quad \frac{T}{\Delta_{0}}\right|_{\text {tric }}=0.31833 .
$$

The line $\alpha=0$ should cross the temperature axis at the BCS point. In this way one reobtains the result in Eq. (2.91) for the BCS critical temperature, as well as the value for the tricritical temperature,

$$
\frac{T_{\text {tric }}}{T_{\mathrm{BCS}}}=0.56149 \text {. }
$$

The results given in this section are valid as long as other possible condensates are neglected. In fact, we shall see that close to the first-order transition of the homogeneous phase the LOFF phase with inhomogeneous gap can be formed.

\section{Gap equation for anisotropic superconductor: One plane wave (Fulde-Ferrell state)}

Let us now consider again the condensate wave function $\Xi(\mathbf{r})$ of Eq. (2.9):

$$
\Xi(\mathbf{r})=\langle\operatorname{vac}|\psi(\mathbf{r}, t) C \psi(\mathbf{r}, t)| \mathrm{vac}\rangle .
$$

Here $|v a c\rangle$ is the ground state. We develop it as follows:

$$
|\mathrm{vac}\rangle=\sum_{N=0}^{\infty} c_{N}|N\rangle
$$

where $N$ is even, the state $|N\rangle$ contains $N / 2$ quark pairs of momenta

$$
\mathbf{p}_{1}=+\mathbf{p}+\mathbf{q}, \quad \mathbf{p}_{2}=-\mathbf{p}+\mathbf{q},
$$

respectively, for up and down species, and the sum also implies an integration over the $\mathbf{p}$ variables and sum over spin. Clearly we have

$$
\begin{aligned}
\Xi(\mathbf{r}) & =\sum_{N, M} c_{N}^{*} c_{M}\langle N|\psi(\mathbf{r}, t) C \psi(\mathbf{r}, t)| M\rangle \\
& =\sum_{N} c_{N}^{*} c_{N+2}\langle N|\psi(\mathbf{r}, t) C \psi(\mathbf{r}, t)| N+2\rangle \\
& =\sum_{N} c_{N}^{*} c_{N+2} e^{2 i \mathbf{q}_{N} \cdot \mathbf{r}}\langle N|\psi(0) C \psi(0)| N+2\rangle .
\end{aligned}
$$

The homogeneous solution discussed in the previous subsection corresponds to the choice (Cooper pairs)

$$
\mathbf{q}_{N}=0 \quad(\text { for all } N) \text {, }
$$

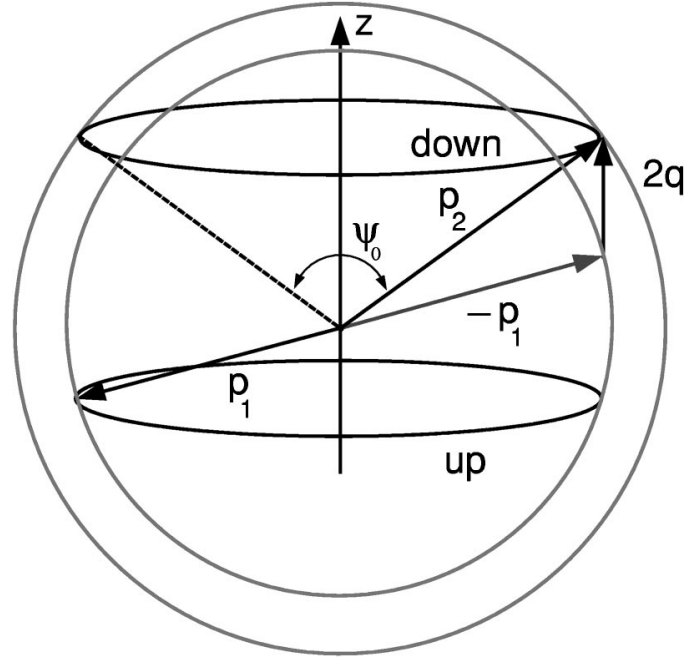

FIG. 4. Kinematics of the Larkin-Ovchinnikov-Fulde-Ferrell (LOFF) state in the case of one-plane-wave behavior of the condensate. The Cooper pair has a total momentum $2 \mathbf{q} \neq 0$.

while $\mathbf{q}_{N} \neq 0$ corresponds to the inhomogeneous state. Let us now assume that the interaction favors the formation of pairs with nonzero total momentum and suppose that the values $\mathbf{q}_{1}, \mathbf{q}_{2}, \ldots, \mathbf{q}_{P}$ are possible. Clearly this hypothesis has to be tested by comparing the values of the free energies for the normal, homogeneous, and inhomogeneous states. In any event, under such hypothesis, since the gap is proportional to $\Xi(\mathbf{r})$, we would get

$$
\Delta(\mathbf{r})=\sum_{m=1}^{P} \Delta_{m} e^{2 i \mathbf{q}_{m} \cdot \mathbf{r}} .
$$

We shall call the phase with $\Delta(\mathbf{r})$ given by Eq. (2.109) inhomogeneous or LOFF superconducting. At the moment we shall assume the existence of a single $\mathbf{q}$ and therefore

$$
\Delta(\mathbf{r})=\Delta e^{2 i \mathbf{q} \cdot \mathbf{r}} .
$$

This is the simplest hypothesis, the one considered by Fulde and Ferrell (1964) (see Fig. 4). It is often called FF state. The paper by Larkin and Ovchinnikov (1964) examines the more general case of Eq. (2.109); we shall come to it below. The assumption of Eq. (2.106) with $q$ $\neq 0$ produces a shift in energy:

$$
\begin{aligned}
\xi_{\mathbf{p}} \pm \delta \mu & =\mathbf{v}_{F} \cdot\left(\mathbf{p}-\mathbf{p}_{F}\right) \pm \delta \mu \rightarrow \mathbf{v}_{F} \cdot\left(\mathbf{p} \mp \mathbf{q}-\mathbf{p}_{F}\right) \pm \delta \mu \\
& =\xi_{\mathbf{p}} \pm \bar{\mu}_{\mathbf{p}},
\end{aligned}
$$

with

$$
\bar{\mu}_{\mathbf{p}}=\delta \mu-\mathbf{q} \cdot \mathbf{v}_{F}=\delta \mu-q v_{F} \cos \theta,
$$

where the upper and lower signs refer to the $d$ and $u$ quasiparticles, respectively. Using the analogous result for a hole with field $\psi^{c}(-\vec{p})$, one can follow the same steps leading to Eq. (2.44) from Eq. (2.38); therefore the gap equation is still given by Eq. (2.44), but now the quasiparticle occupation numbers are

$$
n_{u}(\mathbf{p})=\frac{1}{e^{\left(\epsilon+\bar{\mu}_{\mathbf{p}}\right) / T}+1} \quad \text { and } n_{d}(\mathbf{p})=\frac{1}{e^{\left(\epsilon-\bar{\mu}_{\mathbf{p}}\right) / T}+1} .
$$


TABLE I. The three blocking regions, where we have defined $\cos \theta_{ \pm}=z_{q}(1 \pm \Delta / \delta \mu)$.

\begin{tabular}{ccc}
\hline \hline Region & Definition & Domain of integration in $\cos \theta$ \\
\hline E & $q v_{F}-\delta \mu \leqslant-\Delta$ & $(-1,+1)$ \\
S & $-\Delta \leqslant q v_{F}-\delta \mu \leqslant+\Delta$ & $\left(-1, \cos \theta_{-}\right)$ \\
D & $q v_{F}-\delta \mu \geqslant+\Delta$ & $\left(-1, \cos \theta_{-}\right) \cup\left(\cos \theta_{+},+1\right)$ \\
\hline \hline
\end{tabular}

By Eq. (2.113), using the gap equation for a BCS superconductor with gap $\Delta_{0}$, one writes the gap equation for the inhomogeneous superconductor as

$$
\frac{g \rho}{2} \ln \frac{\Delta_{0}}{\Delta}=\frac{g}{2} \int \frac{d^{3} p}{(2 \pi)^{3}}\left[n_{u}(\mathbf{p})+n_{d}(\mathbf{p})\right] .
$$

In contrast to the case of equal chemical potentials $(\delta \mu=0)$, when there is a phase space reduction at $T$ $\neq 0$, now at $T=0$ the blocking factors also reduce the phase space available for pairing. As a matter of fact the gap equation at $T=0$ reads

$$
\begin{aligned}
\frac{g \rho}{2} \ln \frac{\Delta_{0}}{\Delta} & =\frac{g}{2} \int \frac{d^{3} p}{(2 \pi)^{3}} \frac{1}{\epsilon(\mathbf{p}, \Delta)}\left[\theta\left(-\epsilon-\bar{\mu}_{\mathbf{p}}\right)+\theta\left(-\epsilon+\bar{\mu}_{\mathbf{p}}\right)\right] \\
& =\frac{g \rho}{2} \int_{B R} \frac{d \Omega_{\mathbf{p}}}{4 \pi} \operatorname{arcsinh} \frac{C(\theta)}{\Delta},
\end{aligned}
$$

where

$$
C(\theta)=\sqrt{q^{2} v_{F}^{2}\left(z_{q}-\cos \theta\right)^{2}-\Delta^{2}}
$$

and

$$
z_{q}=\frac{\delta \mu}{q v_{F}}=\cos \frac{\psi_{0}}{2}
$$

where $\psi_{0}$ is the angle depicted in Fig. 4. The angular integration is not over the whole Fermi surface, but only over the region defined by $\epsilon(\mathbf{p}, \Delta)<\left|\bar{\mu}_{\mathbf{p}}\right|$, or

$$
q^{2} v_{F}^{2}\left(z_{q}-\cos \theta\right)^{2}>\Delta^{2} .
$$

Notice that there are no solutions to this inequality for $q v_{F}+\delta \mu \leqslant \Delta$ [compare with Eq. (2.52)]. Analyzing this inequality in terms of $\cos \theta$ we see that there are three regions, obtained by comparing $q v_{F}-\delta \mu$ to $\pm \Delta$. The regions are characterized by different domains of angular integration. They are displayed in Table I. As pointed out by Fulde and Ferrell (1964), the blocking regions correspond to regions in momentum space where fermions do not pair. In regions $\mathrm{E}$ and $\mathrm{S}$ fermions of one type (for instance spin-up) do not pair, whereas in region $\mathrm{D}$ fermions of both types do not pair. The effect of the blocking regions is to reduce the phase space where pairing is possible. The complementary phase space, where pairing is possible, will therefore be called the pairing region. It is formed by two rings that, loosely speaking, are around the two circles of Fig. 4. Since pairing is possible not only on the Fermi surface, but also for modes just below and above it, each ring has a toroidal shape. $\psi_{0}=2 \arccos \left(z_{q}\right)$ is the aperture of the cone, with the vertex at the origin of the spheres, intersecting the Fermi surfaces along the rings.
Once the integration domain is fixed, the remaining integral in $\cos \theta$ is trivial, and the result can be expressed for the three cases in the following uniform way:

$$
\ln \frac{\Delta_{0}}{\Delta}=\frac{\Delta}{2 q v_{F}}\left[G\left(\frac{q v_{F}+\delta \mu}{\Delta}\right)+G\left(\frac{q v_{F}-\delta \mu}{\Delta}\right)\right],
$$

where the function $G(x)$ is defined as follows:

$$
\begin{aligned}
& G(x)=x \quad \operatorname{arccosh}(x)-\sqrt{x^{2}-1}, \quad|x|>1, \\
& G(x)=0, \quad|x|<1, \\
& G(x)=-G(-x), \quad x<0 .
\end{aligned}
$$

The reduction of the available phase space implies a reduction of the gap, which leads us to expect in general smaller gaps than in the homogeneous case. In particular, we see from Eq. (2.115) that with increasing $\delta \mu$ the effect of the blocking terms increases; eventually a phase transition to the normal phase occurs when $\delta \mu$ approaches a maximum value $\delta \mu_{2}$. Therefore the anisotropic superconducting phase can exist only in a window:

$$
\delta \mu_{1}<\delta \mu<\delta \mu_{2}
$$

We expect that $\delta \mu_{1}$ is near the Chandrasekhar-Clogston (Chandrasekhar, 1962; Clogston, 1962) limit $\Delta_{0} / \sqrt{2}$ because Eq. (2.63) shows that near this point the difference in energy between the isotropic superconducting and the normal phases is small and we might expect the LOFF state to correspond to the real ground state. This guess and the gap equation will be discussed below. For the moment we determine $\delta \mu_{2}$. For $\delta \mu \rightarrow \delta \mu_{2}$ the gap $\Delta$ $\rightarrow 0$, and in blocking regions $\mathrm{E}$ and $\mathrm{D}$ the domain of integration in $\cos \theta$ is $(-1,1)$ (the region $\mathrm{S}$ disappears in the limit). Expanding the function $G(x)$ for $x \rightarrow \infty$ we get from Eq. (2.119)

$\ln \frac{\Delta_{0}}{\Delta}=-1+\frac{1}{2} \frac{\delta \mu}{q v_{F}} \ln \frac{q v_{F}+\delta \mu}{q v_{F}-\delta \mu}-\frac{1}{2} \ln \frac{\Delta^{2}}{4\left(q^{2} v_{F}^{2}-\delta \mu^{2}\right)}$,

which can be rewritten as

$$
\begin{aligned}
\alpha\left(q v_{F}, \delta \mu\right)= & -1+\frac{1}{2} \frac{\delta \mu}{q v_{F}} \ln \frac{q v_{F}+\delta \mu}{q v_{F}-\delta \mu} \\
& -\frac{1}{2} \ln \frac{\Delta_{0}^{2}}{4\left(q^{2} v_{F}^{2}-\delta \mu^{2}\right)}=0 .
\end{aligned}
$$

In terms of the dimensionless variables

$$
y=\frac{\delta \mu}{\Delta_{0}}, \quad z=\frac{q v_{F}}{\Delta_{0}},
$$

the condition $\alpha=0$ is equivalent to the equation

$$
y+z=\frac{e}{2}\left(\frac{z+y}{z-y}\right)^{(z-y) / 2 z} .
$$

The critical line is plotted in Fig. 5.

Notice that Eq. (2.122) can also be written in the form 


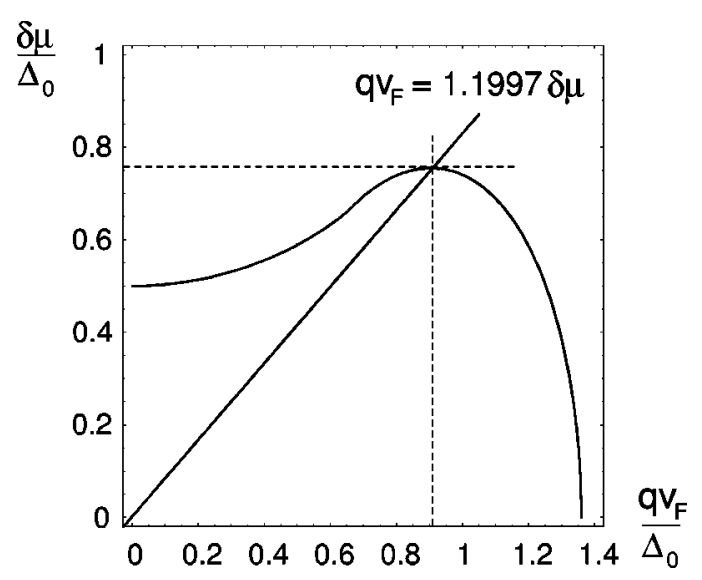

FIG. 5. The critical line for the LOFF phase at $T=0$ in the plane $\left(q v_{F} / \Delta_{0}, \delta \mu / \Delta_{0}\right)$. The line determining $q v_{F}$ as a function of $\delta \mu_{2}$ is also given.

$$
\begin{aligned}
\ln \frac{\Delta_{0}}{2 \delta \mu} & =\frac{1}{2} f_{0}\left(\frac{q v_{F}}{\delta \mu}\right) \\
& =-1+\frac{1}{2} \frac{\delta \mu}{q v_{F}} \ln \frac{q v_{F}+\delta \mu}{q v_{F}-\delta \mu}-\frac{1}{2} \ln \frac{\delta \mu^{2}}{\left(q^{2} v_{F}^{2}-\delta \mu^{2}\right)}
\end{aligned}
$$

where

$$
f_{0}(x)=\int_{-1}^{+1} d u \ln (1+x u) .
$$

We can fix $q$ by minimizing the function $\alpha$ with respect to it. This is equivalent to minimizing the grand potential close to the second-order phase transition. The transition occurs at a value of $x$ satisfying the equation

$$
x=\operatorname{coth} x,
$$

i.e., at

$$
x=\frac{q v_{F}}{\delta \mu_{2}}=1.1997 \equiv x_{2} .
$$

This result can also be obtained from Fig. 5, intersecting the curve $\alpha=0$ at its maximum value $\delta \mu_{2} / \Delta_{0}$ with a straight line passing through the origin.

The value of $\delta \mu$ at which the transition occurs is obtained by substituting this value into Eq. (2.126) and solving for $\delta \mu_{2}$. One gets in this way

$$
\delta \mu_{2}=0.754 \Delta_{0} \text {. }
$$

Since $\delta \mu_{2}>\delta \mu_{1} \approx 0.71 \Delta_{0}$, there exists a window of values of $\delta \mu$ where LOFF pairing is possible. We shall prove below, using the Landau-Ginzburg approach, that the phase transition for the one-plane-wave condensate at $T=0$ and $\delta \mu=\delta \mu_{2}$ is second order.

\section{GINZBURG-LANDAU APPROXIMATION}

The condensate wave function acts as an order parameter characterized by its nonvanishing value in the superconducting phase. At the second-order phase transi-

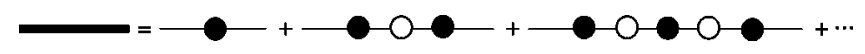

FIG. 6. Ginzburg-Landau expansion of the propagator. The lines represent alternatively $\mathbf{G}_{\mathbf{0}}^{-}$and $\mathbf{G}_{\mathbf{0}}^{+}$, see Eq. (3.1). Full and empty circles represent $\Delta^{*}$ and $\Delta$, respectively.

tion it vanishes and we can apply the general GinzburgLandau approximation (Ginzburg and Landau, 1950). We begin by performing the Ginzburg-Landau expansion at $T=0$ for a general inhomogeneous gap function (Larkin and Ovchinnikov, 1964; Bowers and Rajagopal, 2002). From this we shall derive the grand potential measured with respect to the normal state, and we shall evaluate it explicitly for several cases. Next, in Sec. III.D we shall perform an analogous expansion at $T \neq 0$ around the tricritical point that we have shown to exist in Sec. II.B (Alexander and McTague, 1978; Buzdin and Kachkachi, 1997; Houzet et al., 1999, 2002; Houzet and Buzdin, 2000; Combescot and Mora, 2002). In this discussion, we shall follow Combescot and Mora (2002). These authors have made a rather general analysis with the conclusion that in the generic case the favored state corresponds to a pair of antipodal wave vectors.

\section{A. Gap equation in the Ginzburg-Landau approach}

We begin this section by considering the GinzburgLandau expansion of the Nambu-Gor'kov equations. Let us perform an expansion in $\Delta$ of the propagator $\mathbf{F}$ in Eq. (2.28). This is depicted in Fig. 6. Formally it is written

$$
\begin{aligned}
\mathbf{F}= & +\mathbf{G}_{\mathbf{0}}^{-} \boldsymbol{\Delta}^{*} \mathbf{G}_{\mathbf{0}}^{+}-\mathbf{G}_{\mathbf{0}}^{-} \boldsymbol{\Delta}^{*} \mathbf{G}_{\mathbf{0}}^{+} \Delta \mathbf{G}_{\mathbf{0}}^{-} \boldsymbol{\Delta}^{*} \mathbf{G}_{\mathbf{0}}^{+} \\
& +\mathbf{G}_{\mathbf{0}}^{-} \boldsymbol{\Delta}^{*} \mathbf{G}_{\mathbf{0}}^{+} \boldsymbol{\Delta} \mathbf{G}_{\mathbf{0}}^{-} \boldsymbol{\Delta}^{*} \mathbf{G}_{\mathbf{0}}^{+} \boldsymbol{\Delta} \mathbf{G}_{\mathbf{0}}^{-} \boldsymbol{\Delta}^{*} \mathbf{G}_{\mathbf{0}}^{+}
\end{aligned}
$$

The gap equation has an analogous expansion, schematically depicted in Fig. 7. It has the form

$$
\begin{aligned}
\Delta^{*}= & -i \frac{g}{2} \operatorname{Tr} \int \frac{d E}{2 \pi}\left(\int d \mathbf{r}_{1} G_{0}^{-}\left(\mathbf{r}, \mathbf{r}_{1}\right) \Delta^{*}\left(\mathbf{r}_{1}\right) G_{0}^{+}\left(\mathbf{r}_{1}, \mathbf{r}\right)\right. \\
& -\int \prod_{j=1}^{3} d \mathbf{r}_{\mathbf{j}} G_{0}^{-}\left(\mathbf{r}, \mathbf{r}_{1}\right) \Delta^{*}\left(\mathbf{r}_{1}\right) G_{0}^{+}\left(\mathbf{r}_{\mathbf{1}}, \mathbf{r}_{2}\right) \Delta\left(\mathbf{r}_{2}\right) \\
& \times G_{0}^{-}\left(\mathbf{r}_{2}, \mathbf{r}_{3}\right) \Delta^{*}\left(\mathbf{r}_{3}\right) G_{0}^{+}\left(\mathbf{r}_{3}, \mathbf{r}\right) \\
& +\int \prod_{j=1}^{5} d \mathbf{r}_{\mathbf{j}} G_{0}^{-}\left(\mathbf{r}, \mathbf{r}_{1}\right) \Delta^{*}\left(\mathbf{r}_{1}\right) G_{0}^{+}\left(\mathbf{r}_{\mathbf{1}}, \mathbf{r}_{2}\right) \Delta\left(\mathbf{r}_{2}\right) \\
& \times G_{0}^{-}\left(\mathbf{r}_{2}, \mathbf{r}_{3}\right) \Delta^{*}\left(\mathbf{r}_{3}\right) G_{0}^{+}\left(\mathbf{r}_{3}, \mathbf{r}_{4}\right) \\
& \times \Delta\left(\mathbf{r}_{4}\right) G_{0}^{-}\left(\mathbf{r}_{4}, \mathbf{r}_{5}\right) \Delta^{*}\left(\mathbf{r}_{5}\right) G_{0}^{+}\left(\mathbf{r}_{5}, \mathbf{r}\right)
\end{aligned}
$$

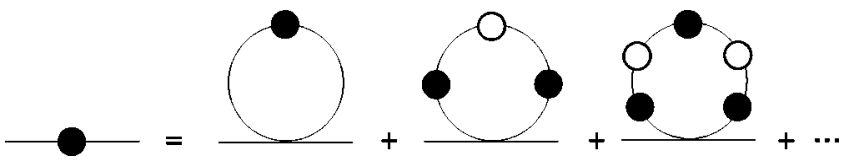

FIG. 7. Ginzburg-Landau expansion of the gap equation. The lines represent alternatively $\mathbf{G}_{\mathbf{0}}^{-}$and $\mathbf{G}_{\mathbf{0}}^{+}$; see Eq. (3.2). Full and empty circles represent $\Delta^{*}$ and $\Delta$, respectively. 
Substituting Eq. (2.109) we get

$$
\begin{aligned}
\Delta_{n}^{*}= & \left(\sum_{k} \Pi\left(\mathbf{q}_{\mathbf{k}}, \mathbf{q}_{\mathbf{n}}\right) \Delta_{k}^{*} \delta\left(\mathbf{q}_{\mathbf{k}}-\mathbf{q}_{\mathbf{n}}\right)\right. \\
& +\sum_{k, \ell, m} J\left(\mathbf{q}_{\mathbf{k}}, \mathbf{q}_{\ell}, \mathbf{q}_{\mathbf{m}}, \mathbf{q}_{\mathbf{n}}\right) \Delta_{k}^{*} \Delta_{\ell} \Delta_{m}^{*} \delta\left(\mathbf{q}_{\mathbf{k}}-\mathbf{q}_{\ell}+\mathbf{q}_{\mathbf{m}}-\mathbf{q}_{\mathbf{n}}\right) \\
& +\sum_{k, \ell, m, j, i} K\left(\mathbf{q}_{\mathbf{k}}, \mathbf{q}_{\ell}, \mathbf{q}_{\mathbf{m}} \mathbf{q}_{\mathbf{j}}, \mathbf{q}_{\mathbf{i}}, \mathbf{q}_{\mathbf{n}}\right) \Delta_{k}^{*} \Delta_{\ell} \Delta_{m}^{*} \Delta_{j} \Delta_{i}^{*} \\
& \left.\times \delta\left(\mathbf{q}_{\mathbf{k}}-\mathbf{q}_{\ell}+\mathbf{q}_{\mathbf{m}}-\mathbf{q}_{\mathbf{j}}+\mathbf{q}_{\mathbf{i}}-\mathbf{q}_{\mathbf{n}}\right)\right)
\end{aligned}
$$

Here $\delta\left(\mathbf{q}_{\mathbf{k}}-\mathbf{q}_{\mathbf{n}}\right)$ means the Kronecker delta: $\delta_{n, k}$ and

$\Pi\left(\mathbf{q}_{1}, \mathbf{q}_{2}\right)$

$$
=+\frac{i g \rho}{2} \int \frac{d \hat{\mathbf{w}}}{4 \pi} \int_{-\delta}^{+\delta} d \xi \int_{-\infty}^{+\infty} \frac{d E}{2 \pi} \prod_{i=1}^{2} f_{i}(E, \delta \mu,\{\mathbf{q}\}),
$$

$J\left(\mathbf{q}_{1}, \mathbf{q}_{2}, \mathbf{q}_{3}, \mathbf{q}_{4}\right)$

$$
=+\frac{i g \rho}{2} \int \frac{d \hat{\mathbf{w}}}{4 \pi} \int_{-\delta}^{+\delta} d \xi \int_{-\infty}^{+\infty} \frac{d E}{2 \pi} \prod_{i=1}^{4} f_{i}(E, \delta \mu,\{\mathbf{q}\}),
$$

\section{$K\left(\mathbf{q}_{1}, \mathbf{q}_{2}, \mathbf{q}_{3}, \mathbf{q}_{4}, \mathbf{q}_{5}, \mathbf{q}_{6}\right)$}

$$
=+\frac{i g \rho}{2} \int \frac{d \hat{\mathbf{w}}}{4 \pi} \int_{-\delta}^{+\delta} d \xi \int_{-\infty}^{+\infty} \frac{d E}{2 \pi} \prod_{i=1}^{6} f_{i}(E, \delta \mu,\{\mathbf{q}\}) .
$$

We have put $\mathbf{w} \equiv v_{F} \hat{\mathbf{w}}$ and

$$
\begin{aligned}
f_{i}(E & , \delta \mu,\{\mathbf{q}\}) \\
& =\frac{1}{E+i \epsilon \operatorname{sgn} E-\delta \mu+(-1)^{i}\left[\xi-2 \sum_{k=1}^{i}(-1)^{k} \mathbf{w} \cdot \mathbf{q}_{\mathbf{k}}\right]} .
\end{aligned}
$$

Moreover, the condition

$$
\sum_{k=1}^{M}(-1)^{k} \mathbf{q}_{\mathbf{k}}=0
$$

holds, with $M=2,4,6$, respectively, for $\Pi, J$, and $K$.

For $\Pi(q) \equiv \Pi(\mathbf{q}, \mathbf{q})$ one gets

$$
\begin{aligned}
\Pi(q)= & \frac{i g \rho}{2} \int \frac{d \hat{\mathbf{w}}}{4 \pi} \int_{-\delta}^{+\delta} d \xi \int_{-\infty}^{+\infty} \frac{d E}{2 \pi} \\
& \times \frac{1}{(E+i \epsilon \operatorname{sgn} E-\bar{\mu})^{2}-\xi^{2}},
\end{aligned}
$$

where $\bar{\mu}=\delta \mu-v_{F} \mathbf{q} \cdot \hat{w}$ is defined in Eq. (2.112) and is identical to the function $C(\theta)$ of Eq. (2.116) with $\Delta$ $=0$. In performing the energy integration in Eq. (3.9) we use the fact that there are contributions only for $|\xi|$ $>|\bar{\mu}|$. Using the gap equation for the homogeneous pairing to get rid of the cutoff $\delta$ we obtain the result

$$
\begin{aligned}
\Pi(q)= & +\frac{g \rho}{2}\left[1+\frac{1}{2} \ln \frac{\Delta_{0}^{2}}{4\left|\left(q v_{F}\right)^{2}-\delta \mu^{2}\right|}\right. \\
& \left.-\frac{\delta \mu}{2 q v_{F}} \ln \left|\frac{q v_{F}+\delta \mu}{q v_{F}-\delta \mu}\right|\right] .
\end{aligned}
$$

$\Pi(q)$ can be rewritten in terms of the function $\alpha$ introduced in Eq. (2.123) as follows:

$$
\alpha(q)=2 \frac{1-\Pi(q)}{g \rho} .
$$

Clearly the gap equation in the Ginzburg-Landau limit, $1=\Pi(q)$, coincides with Eq. (2.123), which was obtained in the one-plane-wave hypothesis. The reason is that, since $\Pi$ depends only on $|\mathbf{q}|$, it assumes the same value for all the crystalline configurations; therefore $\Pi$ does not depend on the crystalline structure of the condensate, and the transition point we determined in Sec. II.C is universal.

For the evaluation of $J$ and $K$ we have to specialize to the different LOFF condensate choices. This will be discussed below.

\section{B. Grand potential}

The grand potential $\Omega$ is given in the GinzburgLandau approximation by

$$
\begin{aligned}
\Omega= & -\frac{1}{g}\left(\sum_{k, n=1}^{P}\left[\Pi\left(\mathbf{q}_{\mathbf{k}}, \mathbf{q}_{\mathbf{n}}\right)-1\right] \Delta_{k}^{*} \Delta_{n} \delta_{\mathbf{q}_{\mathbf{k}}-\mathbf{q}_{\mathbf{n}}}\right. \\
& +\frac{1}{2} \sum_{k, \ell, m, n=1}^{P} J\left(\mathbf{q}_{\mathbf{k}}, \mathbf{q}_{\ell}, \mathbf{q}_{\mathbf{m}}, \mathbf{q}_{\mathbf{n}}\right) \Delta_{k}^{*} \Delta_{\ell} \Delta_{m}^{*} \Delta_{n} \\
& \times \delta_{\mathbf{q}_{k}-\mathbf{q}_{\ell}+\mathbf{q}_{\mathbf{m}}-\mathbf{q}_{\mathbf{n}}}+\frac{1}{3} \sum_{k, \ell, m, j, i, n=1}^{P} K\left(\mathbf{q}_{\mathbf{k}}, \mathbf{q}_{\ell}, \mathbf{q}_{\mathbf{m}}, \mathbf{q}_{\mathbf{j}}, \mathbf{q}_{\mathbf{i}}, \mathbf{q}_{\mathbf{n}}\right) \\
& \left.\times \Delta_{k}^{*} \Delta_{\ell} \Delta_{m}^{*} \Delta_{j} \Delta_{i}^{*} \Delta_{n} \delta_{\mathbf{q}_{\mathbf{k}}-\mathbf{q}_{\ell}+\mathbf{q}_{\mathbf{m}}-\mathbf{q}_{\mathbf{j}}+\mathbf{q}_{\mathbf{i}}-\mathbf{q}_{\mathbf{n}}}\right)
\end{aligned}
$$

where $P$ is the number of independent plane waves in the condensate. Let us assume that

$$
\Delta_{k}=\Delta_{k}^{*}=\Delta \quad(\text { for any } k),
$$

so that we can rewrite Eq. (3.12) as

$$
\frac{\Omega}{\rho}=P \frac{\alpha}{2} \Delta^{2}+\frac{\beta}{4} \Delta^{4}+\frac{\gamma}{6} \Delta^{6},
$$

where $\alpha$ is related to $\Pi(q)$ through Eq. (3.11) and

$$
\begin{aligned}
\beta= & -\frac{2}{g \rho} \sum_{k, \ell, m, n=1}^{P} J\left(\mathbf{q}_{\mathbf{k}}, \mathbf{q}_{\ell}, \mathbf{q}_{\mathbf{m}}, \mathbf{q}_{\mathbf{n}}\right) \delta_{\mathbf{q}_{\mathbf{k}}-\mathbf{q}_{\ell}+\mathbf{q}_{\mathbf{m}}-\mathbf{q}_{\mathbf{n}},} \\
\gamma= & -\frac{2}{g \rho} \sum_{k, \ell, m, j, i, n=1}^{P} K\left(\mathbf{q}_{\mathbf{k}}, \mathbf{q}_{\ell}, \mathbf{q}_{\mathbf{m}}, \mathbf{q}_{\mathbf{j}}, \mathbf{q}_{\mathbf{i}}, \mathbf{q}_{\mathbf{n}}\right) \\
& \times \delta_{\mathbf{q}_{\mathbf{k}}-\mathbf{q}_{\ell}+\mathbf{q}_{\mathbf{m}}-\mathbf{q}_{\mathbf{j}}+\mathbf{q}_{\mathbf{i}}-\mathbf{q}_{\mathbf{n}} .}
\end{aligned}
$$


It follows from the discussion in Sec. II.C that, at $\delta \mu$ $=\delta \mu_{2}, \alpha$ vanishes; moreover, $\alpha<0$ for $\delta \mu<\delta \mu_{2}$ [see Eq. (3.23) below]. Exactly as we did in Sec. II.B, we distinguish different cases:

(1) $\beta>0, \gamma>0$. In this case $\Delta=0$ is a maximum for $\Omega$, and the minima occur at the points given in Eq. (2.96), which now reads

$$
\Delta^{2}=\frac{-\beta+\sqrt{\beta^{2}-4 P \alpha \gamma}}{2 \gamma} \text {. }
$$

Near the transition point one has

$$
\Delta^{2} \approx-\frac{P \alpha}{\beta} .
$$

A phase transition occurs when $\alpha=0$, i.e., at $\delta \mu$ $=\delta \mu_{2}$. The transition is second order since the gap goes continuously to zero at the transition point.

(2) $\beta<0, \gamma>0$. Both for $\alpha<0$ and for $\alpha>0, \Delta^{2}$ in Eq. (3.17) is a minimum for $\Omega$. In the former case it is the only minimum, as $\Delta=0$ is a maximum; in the latter case it competes with the solution $\Delta=0$. Therefore the LOFF phase can persist beyond $\delta \mu_{2}$, the limit for the single-plane-wave LOFF condensate, up to a maximal value $\delta \mu^{*}$. At $\delta \mu=\delta \mu^{*}$ the free energy vanishes and there are degenerate minima at

$\Delta=0, \quad \Delta^{2}=\frac{-3 \beta}{4 \gamma}$.

The critical point $\delta \mu^{*}$ is obtained by Eq. (2.98), which in the present case can be written as

$\alpha\left(q v_{F}=1.1997 \delta \mu^{*}, \delta \mu^{*}\right)=\frac{3 \beta^{2}}{16 P \gamma}$.

The phase transition from the crystalline to the normal phase at $\delta \mu^{*}$ is first order.

(3) $\beta<0, \gamma<0$. In this case the Ginzburg-Landau expansion (3.14) is inadequate, since $\Omega$ is not bounded from below, and another term $\mathcal{O}\left(\Delta^{8}\right)$ is needed.

In the case $\beta<0, \gamma>0$ we can select the most favored structure by computing the free energy at a fixed value of $\delta \mu$. We choose $\delta \mu=\delta \mu_{2}$ where the Fulde-Ferrell state has a second-order phase transition and $\alpha=0$. There one has

$$
\Delta^{2}=-\frac{\beta}{\gamma}, \quad \frac{\Omega}{\rho}=\frac{\beta^{3}}{12 \gamma^{2}} .
$$

\section{Crystalline structures}

For any crystalline structure the function $\alpha$ in the first term of the Ginzburg-Landau expansion is given by
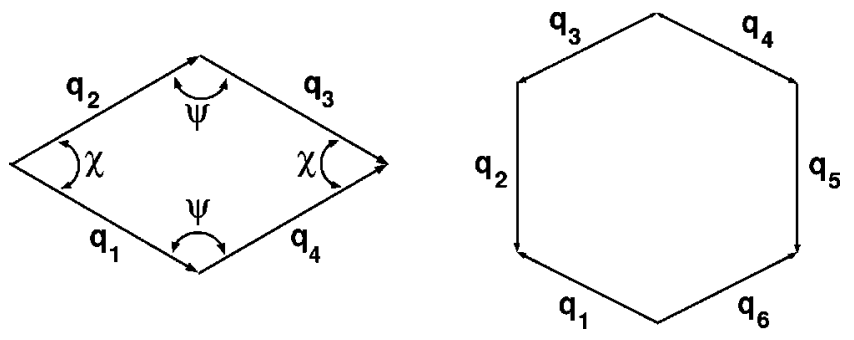

FIG. 8. Rhombic and hexagonal configurations for the vectors $\mathbf{q}_{i}$. The vectors are assumed to be of the same length $q$ and such that $\mathbf{q}_{1}-\mathbf{q}_{2}+\mathbf{q}_{3}-\mathbf{q}_{4}=0$ for the rhombus and $\mathbf{q}_{1}-\mathbf{q}_{2}+\mathbf{q}_{3}$ $-\mathbf{q}_{4}+\mathbf{q}_{5}-\mathbf{q}_{6}=0$ for the hexagon. The vectors need not be all in the same plane.

$$
\begin{aligned}
\alpha & =-1-\frac{1}{2} \ln \frac{\Delta_{0}^{2}}{4\left|\left(q v_{F}\right)^{2}-\delta \mu^{2}\right|}+\frac{\delta \mu}{2 q v_{F}} \ln \left|\frac{q v_{F}+\delta \mu}{q v_{F}-\delta \mu}\right| \\
& =-\ln \frac{\Delta_{0}}{2 \delta \mu}+\frac{1}{2} f_{0}\left(\frac{q v_{F}}{\delta \mu}\right),
\end{aligned}
$$

where we have used Eqs. (2.126), (3.10), and (3.11); $\alpha$ vanishes for $\delta \mu=\delta \mu_{2}$, which characterizes the secondorder transition point at $T=0$, see Eq. (2.123) or Eq. (3.18). Therefore we can write

$$
\alpha=-\frac{\eta}{\delta \mu_{2}},
$$

where

$$
\eta=\delta \mu_{2}-\delta \mu
$$

and we have expanded $\alpha$ around $\delta \mu_{2}$ and used the property of a minimum of $f_{0}(x)$ at $\delta \mu=\delta \mu_{2}$. We observe that, for $\delta \mu<\delta \mu_{2}, \alpha$ is negative; therefore the transition at $T=0$ is always second order if $\beta>0$.

As for the other terms, we can use the results of Appendix A to get the first terms of the Ginzburg-Landau expansion for any crystal structure. The exception is the one-plane-wave case, in which the free energy can be computed to any desired order.

\section{One plane wave}

Using the results of Appendix A for the Fulde-Ferrel one-plane-wave condensate we get
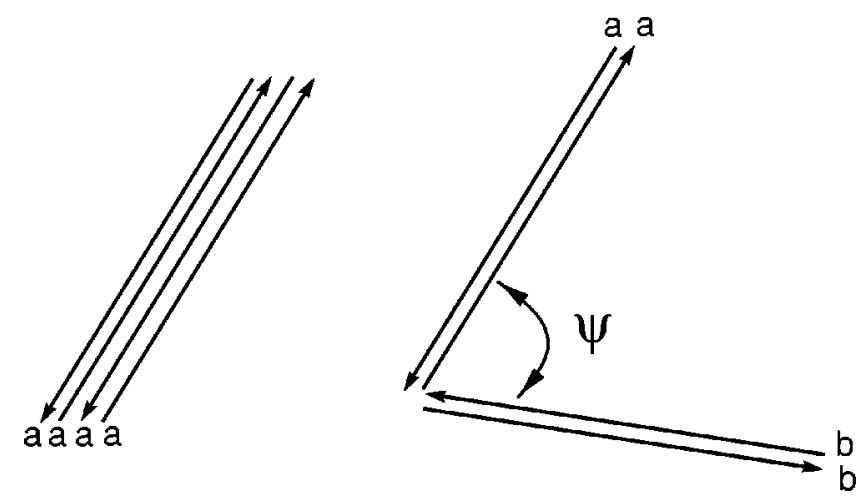

FIG. 9. The two rhombic structures corresponding to the integrals $J_{0}$ and $J_{\psi}$ of Eqs. (3.30). The indices $a$ and $b$ refer to the vectors $\mathbf{q}_{a}$ and $\mathbf{q}_{b}$, respectively. 


$$
\begin{aligned}
& J=J_{0} \equiv-\frac{g \rho}{8} \frac{1}{\left(q v_{F}\right)^{2}-\delta \mu^{2}}, \\
& K=K_{0} \equiv-\frac{g \rho}{64} \frac{\left(q v_{F}\right)^{2}+3 \delta \mu^{2}}{\left[\left(q v_{F}\right)^{2}-\delta \mu^{2}\right]^{3}} .
\end{aligned}
$$

From Eq. (3.25) we get $\left(x_{2}=q v_{F} / \delta \mu_{2}=1.1997\right)$

$$
\begin{aligned}
& \beta=\frac{1}{4 \delta \mu_{2}^{2}\left(x_{2}^{2}-1\right)}=+\frac{0.569}{\delta \mu_{2}^{2}}, \\
& \gamma=\frac{3+x_{2}^{2}}{8 \delta \mu_{2}^{4}\left(x_{2}^{2}-1\right)^{3}}=+\frac{1.637}{\delta \mu_{2}^{4}} .
\end{aligned}
$$

Since $\beta>0$, the $\gamma$ term is ineffective near the transition point and Eq. (3.18) gives

$$
\Delta^{2}=4 \eta\left(x_{2}^{2}-1\right) \delta \mu_{2} \approx 1.757 \eta \delta \mu_{2} .
$$

We can get $\Omega$ from Eq. (3.14) with $P=1$ and using Eqs. (3.23) and (3.26). The result is

$$
\Omega=-\frac{\alpha^{2} \rho}{4 \beta}=-0.439 \rho\left(\delta \mu-\delta \mu_{2}\right)^{2} .
$$

The same result could also be obtained using Eqs. (2.59) and (2.62).

\section{Generic crystals}

In the general case $P \neq 1$, and the evaluation of $J$ and $K$ is more complicated. First, we introduce Feynman parametrizations, then the energy, longitudinal momenta, and angles integrations are performed along the lines sketched in Appendix A, which are based mainly on Bowers and Rajagopal (2002). Next, we have to perform the integration over the Feynman parameters. To do this it is useful to draw two pictures: a rhombus with lines formed by the four vectors appearing in $J\left(\mathbf{q}_{\mathbf{k}}, \mathbf{q}_{\ell}, \mathbf{q}_{\mathbf{m}}, \mathbf{q}_{\mathbf{n}}\right)$ implementing the condition $\mathbf{q}_{\mathbf{k}}-\mathbf{q}_{\ell}+\mathbf{q}_{\mathbf{m}}-\mathbf{q}_{\mathbf{n}}=0$, and a hexagon with lines formed by the six vectors appearing in $K\left(\mathbf{q}_{k}, \mathbf{q}_{\ell}, \mathbf{q}_{\mathbf{m}}, \mathbf{q}_{\mathbf{j}}, \mathbf{q}_{\mathbf{i}}, \mathbf{q}_{\mathbf{n}}\right)$ that satisfy $\mathbf{q}_{\mathbf{k}}-\mathbf{q}_{\ell}+\mathbf{q}_{\mathbf{m}}-\mathbf{q}_{\mathbf{j}}+\mathbf{q}_{\mathbf{i}}$ $-\mathbf{q}_{\mathbf{n}}=0$, see Fig. 8. Note that the rhombus and the hexagon need not be in a plane. The simplest example is provided by two plane waves.

\section{Two plane waves}

In this case $P=2$; let the two vectors be $\mathbf{q}_{\mathbf{a}}, \mathbf{q}_{\mathbf{b}}$, forming an angle $\psi$, a simpler case is provided by an antipodal pair, $\mathbf{q}_{\mathbf{a}}=-\mathbf{q}_{\mathbf{b}}=\mathbf{q}$ and $\psi=\pi$, with

$$
\Delta(\mathbf{r})=2 \Delta \cos 2 \mathbf{q} \cdot \mathbf{r} \text {. }
$$

To get $\beta$ from Eq. (A5) we note that the integral $J$ assumes two different values:

$$
J_{0}=J\left(\mathbf{q}_{\mathbf{a}}, \mathbf{q}_{\mathbf{a}}, \mathbf{q}_{\mathbf{a}}, \mathbf{q}_{\mathbf{a}}\right) \text { and } J_{\psi}=J\left(\mathbf{q}_{\mathbf{a}}, \mathbf{q}_{\mathbf{a}}, \mathbf{q}_{\mathbf{b}}, \mathbf{q}_{\mathbf{b}}\right),
$$

corresponding to Fig. 9. $J_{0}$ has already been computed [see Eq. (3.25)]; on the other hand,

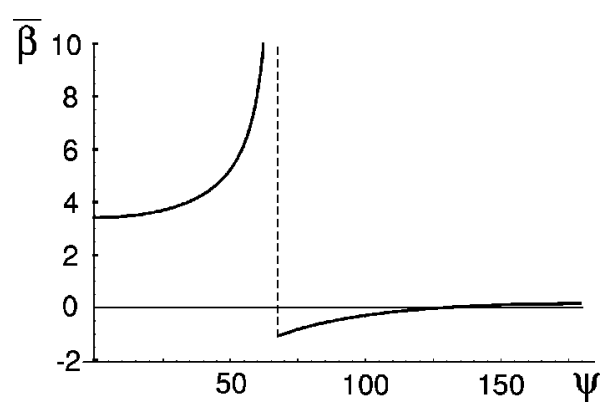

FIG. 10. $\bar{\beta}=\beta \delta \mu_{2}^{2}$ as a function of the opening angle $\psi$ between the two plane-wave vectors $\mathbf{q}_{\mathbf{a}}$ and $\mathbf{q}_{\mathbf{b}} ; \psi=\psi_{0}=67.07^{\circ}$ is the angle defining the LOFF ring; $\bar{\beta}\left(\psi_{0}\right)=-1.138$.

$$
J_{\psi}=-\frac{g \rho}{2 \delta \mu^{2}} \operatorname{Re} \frac{\arctan \frac{x_{2} \sqrt{\cos \psi-1}}{2 x_{2} \sqrt{(\cos \psi-1)\left[2-x_{2}^{2}(1+\cos \psi\right.}}}{2, \cos \psi)]}
$$

which for $\psi=\pi$ gives

$$
J_{\pi}=-\frac{g \rho}{8 \delta \mu_{2}^{2}} .
$$

Using rotation and parity symmetry of the integrals one gets

$$
\beta(\psi)=-\frac{2}{g \rho}\left(2 J_{0}+4 J_{\psi}\right) .
$$

The result for $\beta(\psi)$ as a function of $\psi$ is reported in Fig. 10. In the case of the antipodal pair $(\mathbf{q},-\mathbf{q})$, when $\psi$ $=180^{\circ}$, one gets

$$
\begin{aligned}
\beta=-\frac{2}{g \rho}\left(2 J_{0}+4 J_{\pi}\right) & =\frac{1}{\delta \mu_{2}^{2}}\left(\frac{1}{2\left(x_{2}^{2}-1\right)}-1\right) \\
& =+\frac{0.138}{\delta \mu_{2}^{2}} .
\end{aligned}
$$

For $K$ we have three possibilities (see Fig. 11):

$$
\begin{aligned}
& K_{0}=K\left(\mathbf{q}_{\mathbf{a}}, \mathbf{q}_{\mathbf{a}}, \mathbf{q}_{\mathbf{a}}, \mathbf{q}_{\mathbf{a}}, \mathbf{q}_{\mathbf{a}}, \mathbf{q}_{\mathbf{a}}\right), \\
& K_{1}(\psi)=K\left(\mathbf{q}_{\mathbf{a}}, \mathbf{q}_{\mathbf{a}}, \mathbf{q}_{\mathbf{a}}, \mathbf{q}_{\mathbf{a}}, \mathbf{q}_{\mathbf{b}}, \mathbf{q}_{\mathbf{b}}\right), \\
& K_{2}(\psi)=K\left(\mathbf{q}_{\mathbf{a}}, \mathbf{q}_{\mathbf{a}}, \mathbf{q}_{\mathbf{b}}, \mathbf{q}_{\mathbf{b}}, \mathbf{q}_{\mathbf{b}}, \mathbf{q}_{\mathbf{b}}\right) .
\end{aligned}
$$

Therefore we have

$$
\gamma(\psi)=-\frac{2}{g \rho}\left[2 K_{0}+12 K_{1}(\psi)+6 K_{2}(\psi)\right] .
$$

$K_{0}$ has been already computed in Eq. (3.25), whereas $K_{1}$ and $K_{2}$ can be evaluated using the results given in Appendix A. $\gamma(\psi)$ is plotted in Fig. 12. In the case of the antipodal pair, when $\psi=180^{\circ}$, the result for $\gamma$ is shown in Table II.

Figures 10 and 12 show a divergence at

$$
\psi=\psi_{0}=67.07^{\circ}=2 \arccos \frac{\delta \mu_{2}}{q v_{F}} .
$$

Here $\psi_{0}$ is the opening angle depicted in Fig. 4. In this 

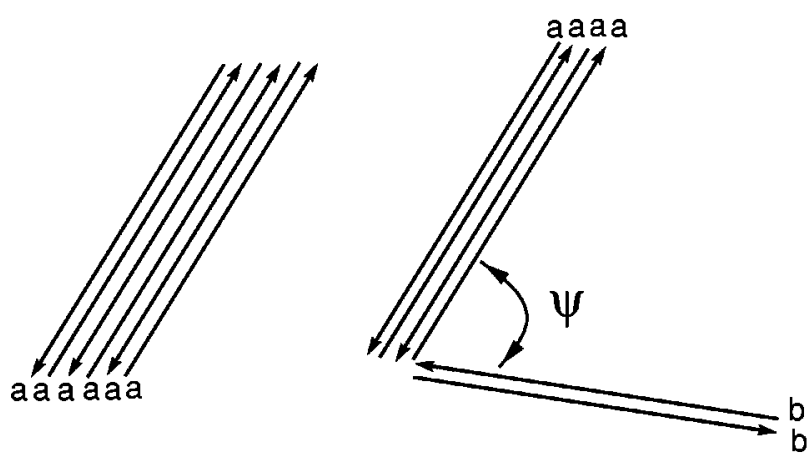

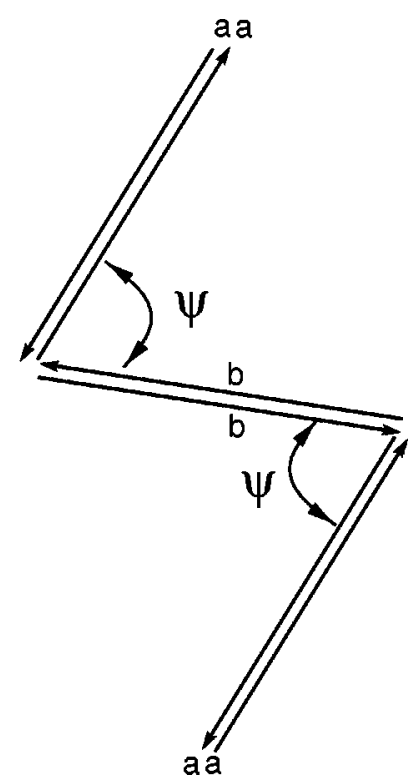

FIG. 11. The three hexagonal structures corresponding to the integrals $K_{0}, K_{1}$, and $K_{2}$ of Eqs. (3.35). The indices $a$ and $b$ refer to the vectors $\mathbf{q}_{a}$ and $\mathbf{q}_{b}$, respectively. case, unlike the one-plane-wave situation, we have two different rings for each Fermi surface. For $\psi>\psi_{0}$ the two rings do not intersect, at $\psi=\psi_{0}$ they are contiguous, while for $\psi<\psi_{0}$ they overlap. The structure with $\psi<\psi_{0}$ is energetically disfavored because, when $\beta$ is large and positive the free energy would be smaller according to Eq. (3.18). According to the discussion of Bowers and Rajagopal (2002), this behavior seems universal, i.e., structures with overlapping rings are energetically disfavored in comparison with structures without overlaps. We shall use this result in Sec. V.D.

For $\psi_{0}<\psi<132^{\circ}, \beta$ is negative. Therefore according to the discussion above we are in the presence of a second-order phase transition ( $\gamma$ is always positive as can be seen from Fig. 12). As is clear from Eq. (3.21), the most favorable case from the energetic point of view occurs when $\gamma$ assumes its smallest value and $|\beta|$ its largest, i.e., at $\psi=\psi_{0}$ when the rings are tangent. The values for this case are reported in Table II.

For comparison, at $\psi=90^{\circ}$ we have $\delta \mu_{2}^{2} \beta\left(90^{\circ}\right)$ $=-0.491$ and $\delta \mu_{2}^{4} \gamma\left(90^{\circ}\right)=1.032$; the first-order transition takes place at $\delta \mu^{*}=0.771 \Delta_{0}$, which is only marginally larger than $\delta \mu_{2}$, and the dimensionless free energy $\bar{\Omega}=\Omega /\left(\rho \Delta_{0}^{2}\right)$ assumes the value $\bar{\Omega}=-0.005$ at $\delta \mu$

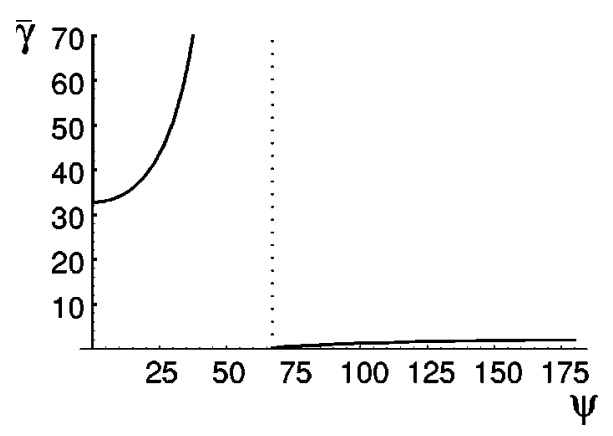

FIG. 12. $\bar{\gamma}=\gamma \delta \mu_{2}^{4}$ as a function of the opening angle $\psi$ between the two plane-wave vectors $\mathbf{q}_{\mathbf{a}}$ and $\mathbf{q}_{\mathbf{b}} ; \psi=\psi_{0}=67.07^{\circ}$ is the angle defining the LOFF ring; $\bar{\gamma}\left(\psi_{0}\right)=0.249$. $=\delta \mu_{2}$, which is larger than the value obtained for $\psi$ $=\psi_{0}$ (see Table II).

\section{Other structures}

One could continue in the same way by considering other structures. An extensive analysis was performed by Bowers and Rajagopal (2002), who considered 23 different crystalline structures. We refer the interested reader to Table I in this paper, as well as to Appendix A, where the technical aspects of integration over the Feynman parameters of the $K$ integrals for more complicated structures are worked out. From our previous discussion we know that the most energetically favored crystals are those which present a first-order phase transition between the LOFF and the normal phase. Among the regular structures, with $\gamma>0$, examined by Bowers and Rajagopal (2002) the favored one seems to be the octahedron $(P=6)$, with $\delta \mu^{*}=3.625 \Delta_{0}$. Special attention, however, should be given to the face-centered cube; we have reported the values of its parameters, as computed by Bowers and Rajagopal (2002), in Table II. We note that $\gamma<0$ for this structure. The condensate in this case is given by

$$
\Delta(\mathbf{r})=\sum_{k=1}^{8} \Delta_{k}(\mathbf{r})=\sum_{k=1}^{8} \Delta \exp \left(2 i q \hat{\mathbf{n}}_{\mathbf{k}} \cdot \mathbf{r}\right),
$$

where $\hat{\mathbf{n}}_{\mathbf{k}}$ are the eight unit vectors defining the vertices of the cube:

$$
\begin{aligned}
& \hat{\mathbf{n}}_{\mathbf{1}}=\frac{1}{\sqrt{3}}(+1,+1,+1), \quad \hat{\mathbf{n}}_{\mathbf{2}}=\frac{1}{\sqrt{3}}(+1,-1,+1), \\
& \hat{\mathbf{n}}_{\mathbf{3}}=\frac{1}{\sqrt{3}}(-1,-1,+1), \quad \hat{\mathbf{n}}_{\mathbf{4}}=\frac{1}{\sqrt{3}}(-1,+1,+1), \\
& \hat{\mathbf{n}}_{\mathbf{5}}=\frac{1}{\sqrt{3}}(+1+, 1,-1), \quad \hat{\mathbf{n}}_{\mathbf{6}}=\frac{1}{\sqrt{3}}(+1,-1,-1),
\end{aligned}
$$


TABLE II. Candidate crystal structures with $P$ plane waves. $\bar{\beta}=\delta \mu_{2}^{2} \beta, \bar{\gamma}=\delta \mu_{2}^{4} \gamma, \bar{\Omega}=\Omega /\left(\rho \Delta_{0}\right)$, with $\rho=p_{F}^{2} /\left(\pi^{2} v_{F}\right)$, is the $($ dimensionless) minimum free energy computed at $\delta \mu=\delta \mu_{2}$, obtained from Eq. (3.21). The phase transition (first order for $\bar{\beta}<0$ and $\bar{\gamma}>0$, second order for $\bar{\beta}>0$ and $\bar{\gamma}>0$ ) occurs at $\delta \mu^{*}$, given by Eq. (3.20) for first-order transitions.

\begin{tabular}{cccccc}
\hline \hline Structure & $P$ & $\bar{\beta}$ & $\bar{\gamma}$ & $\bar{\Omega}_{\min }$ & $\delta \mu^{*} / \Delta_{0}$ \\
\hline Fulde-Ferrell state & 1 & 0.569 & 1.637 & 0 & 0.754 \\
Antipodal plane waves & 2 & 0.138 & 1.952 & 0 & 0.754 \\
Two plane waves $\left(\psi=\psi_{0}\right)$ & 2 & -1.138 & 0.249 & -1.126 & 1.229 \\
Face-centered cube & 8 & -110.757 & -459.242 & & \\
\hline \hline
\end{tabular}

$$
\hat{\mathbf{n}}_{\mathbf{7}}=\frac{1}{\sqrt{3}}(-1,-1,-1), \quad \hat{\mathbf{n}}_{\mathbf{8}}=\frac{1}{\sqrt{3}}(-1,+1,-1) .
$$

Strictly speaking, since both $\beta$ and $\gamma$ are negative, nothing can be said about the cube and one should compute the eighth order in the Ginzburg-Landau expansion, given by $\delta \Delta^{8} / 8$; the transition would be first order if $\delta$ $>0$. However, Bowers and Rajagopal (2002) argue that, given the large value of $\gamma$, this structure would necessarily dominate. Reasonable numerical examples discussed by the authors confirm this guess.

\section{The LOFF phase around the tricritical point; Larkin- Ovchinnikov subspace}

The LOFF phase can be studied analytically around the tricritical point that we considered in Sec. II.B (Buzdin and Kachkachi, 1997; Combescot and Mora, 2002). Here we shall follow the treatment of Combescot and Mora (2002). The tricritical point is the place where one expects the LOFF transition line to start. Close to it one also expects the total pair momentum to vanish. Therefore one can perform simultaneous expansions in the gap parameter and in the total momentum. Starting from the expressions given in Sec. III [see Eqs. (3.4), (3.5), and (3.6)] and proceeding as in Sec. II.B we find

$$
\begin{aligned}
\Omega= & \sum_{\mathbf{q}} \widetilde{\alpha}(\mathbf{q})\left|\Delta_{\mathbf{q}}\right|^{2}+\frac{1}{2} \sum_{\mathbf{q}_{i}} \widetilde{\beta}\left(\mathbf{q}_{i}\right) \Delta_{\mathbf{q}_{1}} \Delta_{\mathbf{q}_{2}}^{*} \Delta_{\mathbf{q}_{3}} \Delta_{\mathbf{q}_{4}}^{*} \\
& +\frac{1}{3} \sum_{\mathbf{q}_{i}} \widetilde{\gamma}\left(\mathbf{q}_{i}\right) \Delta_{\mathbf{q}_{1}} \Delta_{\mathbf{q}_{2}}^{*} \Delta_{\mathbf{q}_{3}} \Delta_{\mathbf{q}_{4}}^{*} \Delta_{\mathbf{q}_{5}} \Delta_{\mathbf{q}_{6}}^{*} .
\end{aligned}
$$

Here we have used momentum conservation in the fourth- and sixth-order terms,

$$
\mathbf{q}_{1}+\mathbf{q}_{3}=\mathbf{q}_{2}+\mathbf{q}_{4}, \quad \mathbf{q}_{1}+\mathbf{q}_{3}+\mathbf{q}_{5}=\mathbf{q}_{2}+\mathbf{q}_{4}+\mathbf{q}_{6},
$$

with

$$
\begin{aligned}
& \widetilde{\alpha}(\mathbf{q})=\alpha+\frac{2}{3} \beta Q^{2}+\frac{8}{15} \gamma Q^{4} \\
& \widetilde{\beta}\left(\mathbf{q}_{i}\right)=\beta+\frac{4}{9} \gamma\left(Q_{1}^{2}+Q_{2}^{2}+Q_{3}^{2}+Q_{4}^{2}+\mathbf{Q}_{1} \cdot \mathbf{Q}_{3}+\mathbf{Q}_{2} \cdot \mathbf{Q}_{4}\right) \\
& \widetilde{\gamma}\left(\mathbf{q}_{i}\right)=\gamma
\end{aligned}
$$

where $\alpha, \beta$, and $\gamma$ were defined in Eqs. (2.86), (2.92), and (2.93), and

$$
\mathbf{Q}=\mathbf{q} v_{F}
$$

In Appendix B we show, as an example, how $\widetilde{\alpha}$ can be obtained from the expansion of $\Pi(q)$ around $\mathbf{Q}=0$. In order to get a coherent expansion we have to consider the modulus of the pair total momentum of the same order as the gap. In fact, as we shall see, the optimal choice for $Q$ turns out to be of order $\Delta$. Correspondingly we have to expand the coefficient of the quadratic term in the gap up to the fourth order in the momentum and the fourth-order term in the gap up to the second order in the momentum. In the form given in Eq. (3.40) we can easily apply the general analysis used around the tricritical point in Sec. II.B. In particular, for vanishing total momenta of the pairs, we are back to the case of the homogeneous superconductor studied in Sec. II.B.

It is interesting to write the expression for the grand potential in configuration space because it shows that around the critical point the minimization problem boils down to solving a differential equation, whereas at a generic point the Ginzburg-Landau equations are integral ones. By Fourier transformation we get from Eq. (3.40)

$$
\begin{aligned}
\Omega= & \int d^{3} \mathbf{r}\left[\alpha|\Delta(\mathbf{r})|^{2}+\frac{2}{3} \beta|\vec{\nabla} \Delta(\mathbf{r})|^{2}+\frac{8}{15} \gamma\left|\vec{\nabla}^{2} \Delta(\mathbf{r})\right|^{2}\right] \\
& +\int d^{3} \mathbf{r}\left[\beta|\Delta(\mathbf{r})|^{4}+\frac{2}{9} \gamma\left[2\left(\vec{\nabla}|\Delta(\mathbf{r})|^{2}\right]^{2}\right.\right. \\
& +3\left[\vec{\nabla} \Delta^{2}(\mathbf{r})\right]\left[\vec{\nabla} \Delta^{* 2}(\mathbf{r})\right]+\frac{1}{8} \gamma \int d^{3} \mathbf{r}|\Delta(\mathbf{r})|^{6} .
\end{aligned}
$$

We recall from Sec. II.B [see Eq. (2.98)] that the firstorder phase transition is given by

$$
\beta_{\mathrm{first}}=-4 \sqrt{\frac{\alpha \gamma}{3}}
$$

with a discontinuity in the gap given by

$$
\Delta^{2}=-4 \frac{\alpha}{\beta}=-\frac{3}{4} \frac{\beta}{\gamma}
$$

[see Eq. (2.99)]. Let us now consider the possibility of a second-order transition in the general LOFF case. Only the quadratic term in the gap is necessary for the discussion, and we have to look at its zero, given by $\widetilde{\alpha}=0$. Since we are considering only the quadratic term we can 


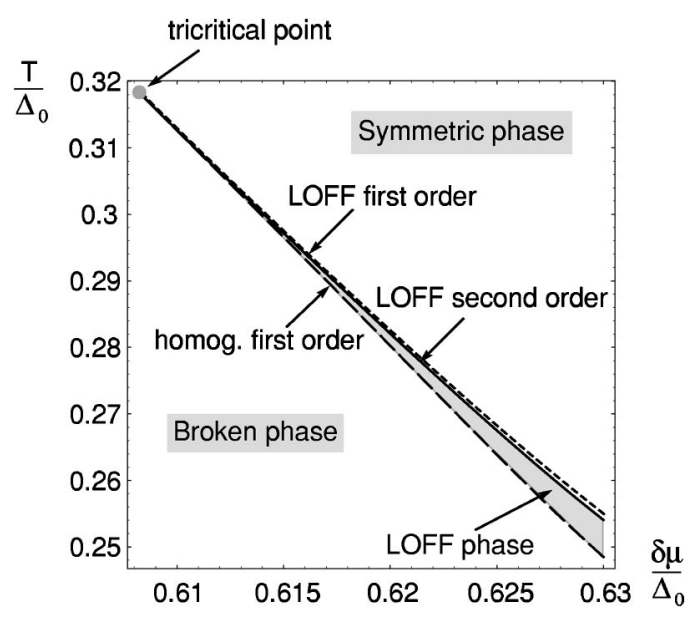

FIG. 13. Transition lines in the plane $\left(\delta \mu / \Delta_{0}, T / \Delta_{0}\right)$ : dashed line, the first-order transition line from the homogeneous broken phase to the symmetric phase; solid line, the second-order transition from the LOFF phase to the symmetric one. The lines start from the tricritical point and end when the LandauGinzburg expansion is no longer valid.

choose an optimal value for $Q^{2}$ by minimizing this term with respect to $|\mathbf{Q}|$. We find

$$
Q^{2}=-\frac{5}{8} \frac{\beta}{\gamma}
$$

requiring $\beta<0$. The corresponding value for $\alpha$ turns out to be

$$
\alpha=\frac{5}{24} \frac{\beta^{2}}{\gamma}
$$

or

$$
\beta_{\text {second }}=-\sqrt{\frac{24}{5} \alpha \gamma} .
$$

The LOFF second-order transition line is higher than the first-order transition line of the homogeneous case, since $\beta_{\text {second }}>\beta_{\text {first }}$ [see Fig. 13, which shows the relevant lines in the plane $\left.\left(\delta \mu / \Delta_{0}, T / \Delta_{0}\right)\right]$. Therefore the secondorder transition to the LOFF case overcomes the firstorder transition to the homogeneous symmetric phase, as can be checked by evaluating the grand potential for the LOFF state along the first-order transition line.

The situation considered above corresponds to the physics of the problem only when the second-order transition is a true minimum of the grand potential. This is not necessarily the case, as we shall explore below.

The second-order term in the grand potential requires that all of the vectors $\mathbf{Q}$ have the same length along the second-order transition line. It is natural to consider the Larkin-Ovchinnikov subspace spanned by plane waves corresponding to momenta with the same length $Q_{0}$ :

$$
\Delta(\mathbf{r})=\sum_{|\mathbf{Q}|=Q_{0}} \Delta_{\mathbf{q}} e^{2 i \mathbf{q} \cdot \mathbf{r}} .
$$

Larkin and Ovchinnikov (1964) restricted their considerations to periodic solutions, but this is not strictly necessary, although the solution found by Combescot and
Mora (2002) is indeed periodic. We shall see that within this subspace the usual LOFF transition (the one corresponding to a single plane wave) is not a stable one. Moreover, there is a first-order transition that overcomes the second-order line. In order that the LOFF line, characterized by Eqs. (3.47) and (3.48), be a true second-order transition, the coefficient of the fourthorder term should be positive. However, in the actual case, the mixed terms in the scalar products of the vectors $\mathbf{Q}$ could change this sign. Combescot and Mora (2002) studied the mixed terms by defining the following quantity:

$$
\begin{aligned}
2 b Q_{0}^{2} \sum_{\mathbf{q}_{i}} \Delta_{\mathbf{q}_{1}} \Delta_{\mathbf{q}_{2}}^{*} \Delta_{\mathbf{q}_{3}} \Delta_{\mathbf{q}_{4}}^{*} \\
\quad=\sum_{\mathbf{q}_{i}} \Delta_{\mathbf{q}_{1}} \Delta_{\mathbf{q}_{2}}^{*} \Delta_{\mathbf{q}_{3}} \Delta_{\mathbf{q}_{4}}^{*}\left(\mathbf{Q}_{1} \cdot \mathbf{Q}_{3}+\mathbf{Q}_{2} \cdot \mathbf{Q}_{4}\right) .
\end{aligned}
$$

Clearly

$$
-1 \leqslant b \leqslant 1,
$$

where $b=1$ is reached in the case of a single plane wave. With this definition and for the optimal choice of $Q_{0}$ [see Eq. (3.47)], for the coefficients appearing in the expression of the grand potential [see Eqs. (3.40) and (3.42)] we get

$$
\widetilde{\alpha}=\alpha-\frac{5}{24} \frac{\beta^{2}}{\gamma}, \quad \widetilde{\beta}=-\frac{1}{9} \beta(5 b+1), \quad \widetilde{\gamma}=\gamma .
$$

Therefore, for any order parameter such that

$$
b<-\frac{1}{5},
$$

it follows that

$$
\widetilde{\beta}<0
$$

and the LOFF line becomes unstable (we recall that close to the second-order line $\beta<0$ ). In fact, since $\widetilde{\alpha}$ $=0$ along this line and $\widetilde{\beta}<0$, a small order parameter is sufficient to make $\Omega$ negative. In other words, we gain by increasing the order parameter as long as the sixthorder term does not grow too much. But then we can make $\Omega=0$ (equal to its value in the symmetric phase) by increasing $\alpha$. Therefore we have a new transition line in the plane $(\alpha, \beta)$ [which is the same in the plane $\left.\left(\delta \mu / \Delta_{0}, T / \Delta_{0}\right)\right]$ to the right of the LOFF line. Combescot and Mora (2002) also shows that necessarily

$$
b \geqslant-\frac{1}{3} \text {. }
$$

Equality is reached for any real order parameter $\bar{\Delta}(\mathbf{r})$. In order to get a better feeling for the parameter $b$ it is useful to consider the quantity.

$2 c Q_{0}^{2} \sum_{\mathbf{q}_{i}} \Delta_{\mathbf{q}_{1}} \Delta_{\mathbf{q}_{2}}^{*} \Delta_{\mathbf{q}_{3}} \Delta_{\mathbf{q}_{4}}^{*}=\sum_{\mathbf{q}_{i}}\left(\mathbf{Q}_{1}-\overline{\mathbf{Q}}_{2}\right)^{2} \Delta_{\mathbf{q}_{1}} \Delta_{\mathbf{q}_{2}}^{*} \Delta_{\mathbf{q}_{3}} \Delta_{\mathbf{q}_{4}}^{*}$.

Expanding the right-hand side of this equation and using $\mathbf{Q}_{1} \cdot \mathbf{Q}_{2}=\mathbf{Q}_{3} \cdot \mathbf{Q}_{4}$ we find 


$$
c=1-b .
$$

Minimizing $b$ is equivalent to maximizing $c$. To this end, it is convenient to have opposite $\mathbf{Q}_{1}$ and $\mathbf{Q}_{2}$ because then $\left(\mathbf{Q}_{1}-\mathbf{Q}_{2}\right)^{2}$ reaches its maximum value equal to $4 Q_{0}^{2}$. In this case we have

$$
\Delta_{\mathbf{q}}^{*}=\Delta_{-\mathbf{q}} .
$$

This is equivalent to requiring that the order parameter be real in configuration space. Of course, it is not necessary that the amplitudes of the different pairs of plane waves be equal.

To proceed further we can introduce a measure of the size of the order parameter,

$$
\frac{1}{\pi^{3}} \int d^{3} \mathbf{r}|\Delta(\mathbf{r})|^{2}=\sum_{\mathbf{q}_{i}} \Delta_{\mathbf{q}_{1}} \Delta_{\mathbf{q}_{2}}^{*} \equiv N_{2} \bar{\Delta}^{2}
$$

and

$$
\begin{aligned}
& \frac{1}{\pi^{3}} \int d^{3} \mathbf{r}|\Delta(\mathbf{r})|^{4}=\sum_{\mathbf{q}_{i}} \Delta_{\mathbf{q}_{1}} \Delta_{\mathbf{q}_{2}}^{*} \Delta_{\mathbf{q}_{3}} \Delta_{\mathbf{q}_{4}}^{*} \equiv N_{4} \bar{\Delta}^{4} \\
& \frac{1}{\pi^{3}} \int d^{3} \mathbf{r}|\Delta(\mathbf{r})|^{6}=\sum_{\mathbf{q}_{i}} \Delta_{\mathbf{q}_{1}} \Delta_{\mathbf{q}_{2}}^{*} \Delta_{\mathbf{q}_{3}} \Delta_{\mathbf{q}_{4}}^{*} \Delta_{\mathbf{q}_{5}} \Delta_{\mathbf{q}_{6}}^{*} \equiv N_{6} \bar{\Delta}^{6} .
\end{aligned}
$$

In the case of a single plane wave we get

$$
N_{2}=N_{4}=N_{6}=1 \text {. }
$$

For a real gap the set of the vectors $\mathbf{Q}_{i}$ is made of $N / 2$ pairs. If all the plane waves have the same amplitude one can show (Combescot and Mora, 2002) that the quantities $N_{2}, N_{4}$, and $N_{6}$ assume the values

$$
\begin{aligned}
& N_{2}=2 N, \quad N_{4}=3 N(N-1), \\
& N_{6}=5 N\left(3 N^{2}-9 N+8\right) .
\end{aligned}
$$

With these notations the grand potential becomes

$$
\begin{aligned}
\Omega= & N_{2}\left(\alpha+\frac{2}{3} \beta Q_{0}^{2}+\frac{8}{15} \gamma Q_{0}^{4}\right) \bar{\Delta}^{2} \\
& +\frac{1}{2} N_{4}\left(\beta+\frac{8}{3} a \gamma Q_{0}^{2}\right) \bar{\Delta}^{4}+\frac{1}{3} N_{6} \gamma \bar{\Delta}^{6},
\end{aligned}
$$

where

$$
a=\frac{b+2}{3}, \quad \frac{1}{3} \leqslant a \leqslant 1 .
$$

Minimizing this expression with respect to $Q_{0}$ we find

$$
Q_{0}^{2}=-\frac{5}{8} \frac{\beta}{\gamma}-\frac{5}{4} a \frac{N_{4}}{N_{2}} \bar{\Delta}^{2} .
$$

Therefore a nonzero solution for $Q_{0}$ is obtained if

$$
\bar{\Delta}^{2} \leqslant-\frac{1}{2 a} \frac{\beta}{\gamma} \frac{N_{2}}{N_{4}}=\bar{\Delta}_{\max }^{2} .
$$

The corresponding expression for $\Omega$ becomes

$$
\begin{aligned}
\Omega= & \alpha N_{2}\left(1-\frac{5}{24} \frac{\beta^{2}}{\alpha \gamma}\right) \bar{\Delta}^{2}+\frac{1}{2} \beta N_{4}\left(1-\frac{5}{3} a\right) \bar{\Delta}^{4} \\
& +\frac{1}{3} \gamma N_{6}\left(1-\frac{5 a^{2}}{2} \frac{N_{4}^{2}}{N_{2} N_{6}}\right) \bar{\Delta}^{6} .
\end{aligned}
$$

In order to have a transition from the symmetric phase we must allow $\Omega / \bar{\Delta}^{2}$ to become negative. The zero of $\Omega$ is reached for

$$
\alpha=\frac{5}{24} \frac{\beta^{2}}{\gamma}+\frac{3}{16} \frac{\beta^{2}}{\gamma} \frac{\left(1-\frac{5 a}{3}\right)^{2}}{\left(\frac{N_{2} N_{6}}{N_{4}^{2}}-\frac{5}{2} a^{2}\right)} .
$$

The value of $\bar{\Delta}$ corresponding to the zero of $\Omega$ is given by

$$
\bar{\Delta}^{2}=-\frac{3}{2} \frac{\beta}{\gamma} \frac{N_{2}}{N_{4}} \frac{\left(1-\frac{5 a}{3}\right)}{\left(\frac{N_{2} N_{6}}{N_{4}^{2}}-\frac{5}{2} a^{2}\right)},
$$

showing that this is a first-order transition. We have shown that it is convenient to have $b$ as small as possible and that the minimum is reached for $b=-1 / 3$ or $a$ $=5 / 9$. In correspondence with this value we have $\bar{\Delta}^{2}$ $\geqslant 0$ and

$$
\alpha>\frac{5}{24} \frac{\beta^{2}}{\gamma} \text {. }
$$

We get

$$
\alpha=\frac{5}{24} \frac{\beta^{2}}{\gamma}+\frac{1}{972} \frac{\beta^{2}}{\gamma} \frac{1}{\frac{N_{2} N_{6}}{N_{4}^{2}}-\frac{125}{162}} .
$$

We see that it is convenient to take $N_{2} N_{6} / N_{4}^{2}$ as small as possible. However, notice that

$$
\frac{N_{2} N_{6}}{N_{4}} \geqslant 1
$$

as follows from the Schwartz inequality

$$
\frac{\left[\int d^{3} \mathbf{r} \bar{\Delta}^{2}(\mathbf{r})\right]\left[\int d^{3} \mathbf{r} \bar{\Delta}^{6}(\mathbf{r})\right]}{\left[\int d^{3} \mathbf{r} \bar{\Delta}^{4}(\mathbf{r})\right]^{2}} \geqslant 1 .
$$

Let us look, for example, at the case of pairs of plane waves with opposite $\mathbf{q}$ but without any further constraints. In this case we get

$$
\frac{N_{2} N_{6}}{N_{4}^{2}}=\frac{5}{9} \frac{3 N^{2}-9 N+8}{(N-1)^{2}} .
$$

This expression has a minimum at $N=2$ (here $N$ is even), where Eq. (3.76) equals 10/9 and then it increases monotonically with $N$ up to the value $15 / 9$. Note that for crystalline structures the situation could be different. For instance, in the case of a cube, the value of $N_{4}$ is not the one given by Eq. (3.64), i.e., not 168, but rather 216. 
However, Combescot and Mora (2002) showed that this expression indeed gets its minimum value for $N=2$. This result is obtained assuming that the plane waves form a generic set of antipodal vectors, which means that the only way to satisfy momentum conservation is through the cancellation of each momentum with the opposite one in the same pair. This excludes special configurations in which other arrangements of vectors could give a zero result. Combescot and Mora (2002) argue that this result should hold in general, but a complete proof is lacking.

The value obtained for $\alpha$ for the two-plane-wave case is

$$
\alpha=\frac{\beta^{2}}{\gamma}\left(\frac{5}{24}+\frac{1}{330}\right)=\frac{\beta^{2}}{\gamma}\left(\frac{5}{36}+3 \times 10^{-3}\right) .
$$

In this case we have three lines, the first-order line just found, the second-order LOFF transition for $\alpha$ $=5 \beta^{2} /(24 \gamma)$, and the first-order transition to the homogeneous broken phase for $\alpha=3 \beta^{2} /(16 \gamma)$. The distance of these two last lines is given by

$$
\frac{\beta^{2}}{\gamma}\left(\frac{3}{16}-\frac{5}{24}\right)=-\frac{1}{48} \frac{\beta^{2}}{\gamma}=-2.1 \times 10^{-2} \frac{\beta^{2}}{\gamma} .
$$

We know that these two lines stay close together up to zero temperature. It turns out that the same is true for the new first-order line as was shown by Matsuo et al. (1998). These results are illustrated in Fig. 13. We notice that whereas in the expansion around the tricritical point the favored state seems to correspond to a pair of plane waves, with a first-order transition between the LOFF and the normal state, at zero temperature one has a second-order phase transition. Therefore the firstorder transition line must change into a second-order line at low temperatures. Matsuo et al. (1998) showed that this happens at a temperature $T / T_{B C S}=0.075$.

It is also interesting to see how things change when the spatial dimensions are varied. In fact it was found by Burkhardt and Rainer (1994) that the first-order transition found previously is second order in two spatial dimensions. This result was confirmed by Buzdin and Tugushev (1983), Buzdin and Polonski (1987), Machida and Nakanishi (1989), and Buzdin and Kachkachi (1997), who showed that the transition is second order in one spatial dimension and can be given an exact solution in terms of the Jacobi elliptic functions. This solution has the property that along the second-order transition line it reduces to the two-plane-wave case considered here. It is very simple to obtain the dependence on the number of dimensions. In fact, the only place where the dimensions enter is in the angular integration as, for instance, in Eq. (B2). In general, this is an average over the $D$-dimensional sphere and we need the following equations for the terms of order $Q^{2}$ and $Q^{4}$, respectively:

$\int \frac{d \hat{\mathbf{w}}}{S_{D}} \hat{w}_{i} \hat{w}_{j}=\frac{\delta_{i j}}{D}$, $\int \frac{d \hat{\mathbf{w}}}{S_{D}} \hat{w}_{i} \hat{w}_{j} \hat{w}_{k} \hat{w}_{l}=\frac{1}{D(D+2)}\left(\delta_{i j} \delta_{k l}+\delta_{i k} \delta_{j l}+\delta_{i l} \delta_{j k}\right)$,

where $S_{D}$ is the surface of the sphere with unitary radius in $D$ dimensions,

$$
S_{D}=\frac{2 \pi^{D / 2}}{\Gamma(D / 2)} .
$$

Therefore terms proportional to $Q^{2}$ are multiplied by $3 / D$ and those proportional to $Q^{4}$ by $15 / D(D+2)$. We get

$$
\begin{aligned}
\widetilde{\alpha}(\mathbf{q})= & \alpha+\frac{2}{D} \beta Q^{2}+\frac{8}{D(D+2)} \gamma Q^{4}, \\
\widetilde{\beta}\left(\mathbf{q}_{i}\right)= & \beta+\frac{4}{3 D} \gamma\left(Q_{1}^{2}+Q_{2}^{2}+Q_{3}^{2}+Q_{4}^{2}+\mathbf{Q}_{1} \cdot \mathbf{Q}_{3}\right. \\
& \left.+\mathbf{Q}_{2} \cdot \mathbf{Q}_{4}\right), \\
\widetilde{\gamma}\left(\mathbf{q}_{i}\right)= & \gamma .
\end{aligned}
$$

Proceeding as before, we find the grand potential evaluated at the optimal value of $Q^{2}$,

$$
\begin{aligned}
\Omega= & \alpha N_{2}\left(1-\frac{D+2}{8 D} \frac{\beta^{2}}{\alpha \gamma}\right) \bar{\Delta}^{2}+\frac{1}{2} \beta N_{4}\left(1-\frac{D+2}{D} a\right) \bar{\Delta}^{4} \\
& +\frac{1}{3} \gamma N_{6}\left(1-\frac{3(D+2)}{2 D} a^{2} \frac{N_{4}^{2}}{N_{2} N_{6}}\right) \bar{\Delta}^{6} .
\end{aligned}
$$

We see from here that the critical dimension for the transition to change from first to second order is at the zero of the second term, that is,

$$
D=\frac{2 a}{1-a},
$$

and for $a=5 / 9, D=2.5$. This means that we have a firstorder transition for $D>2.5$ and a second-order one for $D<2.5$ (remember that $\beta<0$ ). The location of the transition is at $a=5 / 9$ :

$$
\alpha=\frac{D+2}{8 D} \frac{\beta^{2}}{\gamma}+\frac{3}{20} \frac{\beta^{2}}{\gamma} \frac{(2 D-5)^{2}}{D(7 D-10)} .
$$

The value of the gap along the transition line is given by

$$
\Delta^{2}=-\frac{6}{5} \frac{\beta}{\gamma} \frac{2 D-5}{7 D-10}
$$

We see that $\Delta^{2}>0$ for $D>2.5$.

Combescot and Mora (2002) also considered the possibility that solutions around the tricritical point did not belong to the LOFF subspace. In fact, the antipodal solution does not satisfy the Euler-Lagrange equation obtained from Eq. (3.44), due to the nonlinear terms. If these are small it is reasonable to look for solutions close to $\Delta \cos (\mathbf{q} \cdot \mathbf{r})$. This assumption simplifies the problem because the antipodal solution is essentially one dimensional, characterized by the direction of q. Combe- 
scot and Mora (2002) found that the corrections at $\Delta \cos (\mathbf{q} \cdot \mathbf{r})$, expressed in terms of higher harmonics, are indeed very small. Of course this is only a consistency argument, but it is an indication that the choice of the LOFF subspace is a good approximation to the full problem.

To conclude this section, let us say that in our opinion the status of the LOFF phase is not yet settled. Up to now we have considered the Ginzburg-Landau expansion both at $T=0$ and at the tricritical point. The results in the three-dimensional case can be summarized as follows:

- Zero-temperature point: Larkin and Ovchinnikov (1964) found that the favored phase has a gap with a phase modulation $\cos (\mathbf{q} \cdot \mathbf{r})$ corresponding to a structure with two antipodal vectors. This phase and the normal one are separated by a second-order transition line. However, Bowers and Rajagopal (2002) carried out a rather complete study of the possible crystalline structures and argue that the most favorable structure would be the face-centered cube. The transition between the corresponding phase and the normal one should be first order.

- Tricritical point: Buzdin and Kachkachi (1997) studied the nonuniform phase in different dimensions with the result that the space modulation related to a single wave vector [i.e., $\exp (2 i \mathbf{q} \cdot \mathbf{r})]$ is always unfavored. These authors also found that the solution with two antipodal wave vectors is the preferred one. In one and two space dimensions, the transition to the normal state is second order, whereas it is first order in three space dimensions. Analogous results were found by Houzet et al. (1999; see also Agterberg and Yang, 2001), who extended the study to space modulations such as $\cos (q x)+\cos (q y)$ or $\cos (q x)+\cos (q y)$ $+\cos (q z)$. These authors argue that there could be various transition lines at temperatures lower than the tricritical point. Finally, as thoroughly discussed in this section, Combescot and Mora (2002) confirmed the results of Buzdin and Kachkachi (1997).

There have also been numerical investigations of the full phase space. In particular, Burkhardt and Rainer (1994) proved that in the two-dimensional case (layered superconductors) the phase transition from the normal phase to that characterized by two antipodal vectors is second order. The second-order transition line from the phase with a single plane wave to the normal phase was studied by Sarma (1963) and Saint-James et al. (1969). The two-dimensional case for type-II superconductors was studied by Shimahara (1998a), who considered states corresponding to single wave vectors and antipodal pairs together with configurations corresponding to triangular, square, and hexagonal states. It was found that, depending on the temperature, all these states may play a role. Finally, Matsuo et al. (1998), as already mentioned, performed a numerical analysis based on the use of quasiclassical Green's functions. They found that in three dimensions the transition line between the normal phase and the antipodal vectors phase becomes first order at the tricritical point and becomes second order at $T=0.0075 T_{B C S}$. The two-dimensional case will be discussed again in Sec. VI.D.

In conclusion, the question of the preferred nonuniform state cannot yet be considered resolved. The threedimensional case offers a good example. We have seen that there are strong indications that the favored state around the tricritical point is the one corresponding to two antipodal vectors. This being the case, the natural question is: how does the transition line extend down to zero temperature? If at $T=0$ the preferred state were the antipodal pair, then a further tricritical point in the plane $(\delta \mu, T)$ would arise. Recall that the transition is first order at the tricritical point and second order at $T$ $=0$. However, if the conjecture of Bowers and Rajagopal (2002) is correct, the cubic phase would emerge in the path going to $T=0$. A possibility is that one goes from one structure to another in analogy to what was suggested by Shimahara (1998a) for the twodimensional case. The other logical possibility is that the Combescot and Mora result at the tricritical point might be evaded by an exceptional arrangement of the wave vectors as, for instance, in the case of the face-centered cube. Therefore we think that more theoretical work is necessary in order to fill in these gaps in our understanding of the nonuniform superconducting phase.

\section{SUPERCONDUCTIVITY IN QUANTUM CHROMODYNAMICS}

Color superconductivity is an old subject (Collins and Perry, 1975; Barrois, 1977; Frautschi, 1978; Bailin and Love, 1984) that has recently become one of the most fascinating research fields in quantum chromodynamics (QCD). ${ }^{3}$ It offers a clue to the behavior of strong interactions at high baryonic densities, an issue of paramount importance both for the understanding of heavy-ion collisions and for the physics of compact stars. Color superconductivity arises because, for sufficiently high baryon chemical potential $\mu$ and low temperature, the color interaction favors the formation of a quark-quark condensate in the color-antisymmetric channel $\overline{\mathbf{3}}$. In the asymptotic regime it is also possible to understand the structure of the condensates. In fact, consider the matrix element

$$
\left\langle 0\left|\psi_{i a}^{\alpha} \psi_{j b}^{\beta}\right| 0\right\rangle,
$$

where $\alpha, \beta=1,2,3$ are color indices, $a, b=1,2$ are spin

\footnotetext{
${ }^{3}$ The literature of these developments includes Alford et al. (1998, 2000), Rapp et al. (1998), Agasian et al. (1999), Alford, Berges, and Rajagopal (1999), Alford, Rajagopal, and Wilczek (1999), Carter and Diakonov (1999), Pisarski and Rischke (1999a, 2000a), Schafer and Wilczek (1999a, 1999b, 1999c, 1999d), Hong et al. (2000), and Shuster and Son (2000); for reviews, see Hsu (2000), Alford (2001), Hong (2001), and Rajagopal and Wilczek (2001).
} 
indices, and $i, j=1, \ldots, N$ are flavor indices. Its color, spin, and flavor structure are completely fixed by the following considerations:

- Antisymmetry in color indices $(\alpha, \beta)$ in order to have attraction.

- Antisymmetry in spin indices $(a, b)$ in order to get a spin-zero condensate. The isotropic structure of the condensate is favored since a larger portion of the phase space around the Fermi surface is available.

- Antisymmetry in flavor indices $(i, j)$, which, given the structure in color and spin, the Pauli principles requires.

Since the quark and spin momenta in the pair are opposite, it follows that the left-handed quarks can pair only with left-handed quarks and right-handed with righthanded. In the case of three flavors, the favored condensate is

$$
\left\langle 0\left|\psi_{i L}^{\alpha} \psi_{j L}^{\beta}\right| 0\right\rangle=-\left\langle 0\left|\psi_{i R}^{\alpha} \psi_{j R}^{\beta}\right| 0\right\rangle=\Delta \sum_{C=1}^{3} \epsilon^{\alpha \beta C} \epsilon_{i j C} .
$$

This gives rise to the so-called color-flavor-locked (CFL) phase (Alford, Rajagopal, and Wilczek, 1999; Schafer and Wilczek, 1999a). However, at moderate densities other less attractive channels could play a role (Alford et al., 2003). The reason for the name is that simultaneous transformations in color and flavor leave the condensate invariant. In fact, the symmetry-breaking pattern turns out to be

$$
\begin{aligned}
& \mathrm{SU}(3)_{c} \otimes \mathrm{SU}(3)_{L} \otimes \mathrm{SU}(3)_{R} \otimes \mathrm{U}(1)_{B} \\
& \rightarrow \mathrm{SU}(3)_{c+L+R} \otimes Z_{2},
\end{aligned}
$$

where $\mathrm{SU}(3)_{c+L+R}$ is the diagonal subgroup of the three SU(3) groups. Both the chiral group and the color symmetry are broken, but a diagonal SU(3) subgroup remains unbroken. The $Z_{2}$ group arises from the invariance of the condensate when the quark fields are multiplied by -1 . We have 17 broken generators: since there is a broken gauge group, eight of these correspond to eight longitudinal degrees of the gluons, while because the gauge bosons acquire a mass, there are nine NambuGoldstone bosons organized in an octet associated with the breaking of the flavor group and a singlet associated with the breaking of the baryonic number. The effective theory describing the Nambu-Goldstone bosons for the CFL model was studied by Casalbuoni and Gatto (1999).

This is the typical situation when the chemical potential is much larger than the quark masses $m_{u}, m_{d}$, and $m_{s}$ (in these considerations one should discuss densitydependent masses). However, one can ask what happens when the chemical potential is decreased. At intermediate densities asymptotic freedom is no longer supported, but all the model calculations show that one still has a sizable color condensation. In particular, if the chemical potential $\mu$ is much less than the strange-quark mass, one expects the strange quark to decouple, and the corresponding condensate should be

$$
\left\langle 0\left|\psi_{i L}^{\alpha} \psi_{j L}^{\beta}\right| 0\right\rangle=\Delta \epsilon^{\alpha \beta 3} \epsilon_{i j},
$$

since due to the antisymmetry in color the condensate must necessarily choose a direction in color space. Notice that now the symmetry-breaking pattern is completely different from the three-flavor case. In fact, we have

$$
\begin{aligned}
& \mathrm{SU}(3)_{c} \otimes \mathrm{SU}(2)_{L} \otimes \mathrm{SU}(2)_{R} \otimes \mathrm{U}(1)_{B} \\
& \quad \rightarrow \mathrm{SU}(2)_{c} \otimes \mathrm{SU}(2)_{L} \otimes \mathrm{SU}(2)_{R} \otimes \mathrm{U}(1) \otimes Z_{2} .
\end{aligned}
$$

The chiral group remains unbroken, while the original color symmetry group is broken to $\mathrm{SU}(2)_{c}$, with generators $T^{A}$ corresponding to the generators $T^{1}, T^{2}, T^{3}$ of $\mathrm{SU}(3)_{c}$. As a consequence, three gluons remain massless, whereas the remaining five acquire a mass. Even though the original $\mathrm{U}(1)_{B}$ is broken, there is an unbroken global symmetry that plays the role of $\mathrm{U}(1)_{B}$. As for $\mathrm{U}(1)_{A}$, this axial symmetry is broken by anomalies, so that in principle there is no Goldstone boson associated with its being broken by the condensate. However, at high densities, explicit axial symmetry breaking is weak, and therefore there is a light would-be Goldstone boson associated with the breaking of the axial $\mathrm{U}(1)_{A}$. One can construct an effective-field theory to describe the emergence of the unbroken subgroup $\mathrm{SU}(2)_{c}$ and the low-energy excitations, much in the same way as one builds up a chiral effective Lagrangian with effective fields at zero density. For the two-flavor case, such a theory was developed by Casalbuoni et al. (2000) and Rischke et al. (2001).

It is natural to ask what happens in the intermediate region of $\mu$. It turns out that the interesting case is for $\mu \approx M_{s}^{2} / \Delta$. To understand this point, let us consider the case of two fermions, one massive, $m_{1}=M_{s}$, and the other one massless, both at the same chemical potential $\mu$. The Fermi momenta are of course different:

$$
p_{F_{1}}=\sqrt{\mu^{2}-M_{s}^{2}}, \quad p_{F_{2}}=\mu .
$$

The grand potential for the two unpaired fermions is

$$
\begin{aligned}
\Omega_{\text {unpair. }}= & 2 \int_{0}^{p_{F_{1}}} \frac{d^{3} p}{(2 \pi)^{3}}\left(\sqrt{\mathbf{p}^{2}+M_{s}^{2}}-\mu\right) \\
& +2 \int_{0}^{p_{F_{2}}} \frac{d^{3} p}{(2 \pi)^{3}}(|\mathbf{p}|-\mu) .
\end{aligned}
$$

For the two fermions to pair they have to reach some common momentum $p_{\text {comm }}^{F}$, and the corresponding grand potential is

$$
\begin{aligned}
\Omega_{\text {pair. }}= & 2 \int_{0}^{p_{\text {comm }}^{F}} \frac{d^{3} p}{(2 \pi)^{3}}\left(\sqrt{\mathbf{p}^{2}+M_{s}^{2}}-\mu\right) \\
& +2 \int_{0}^{p_{\text {comm }}^{F}} \frac{d^{3} p}{(2 \pi)^{3}}(|\vec{p}|-\mu)-\frac{\mu^{2} \Delta^{2}}{4 \pi^{2}},
\end{aligned}
$$

where the last term is the energy necessary for the condensation of a fermion pair [see Eq. (2.71)]. The com- 
mon momentum $p_{\text {comm }}^{F}$ can be determined by minimizing $\Omega_{\text {pair. }}$ with respect to $p_{\text {comm }}^{F}$. The result (expanding in $\left.M_{s}\right)$ is

$$
p_{\mathrm{comm}}^{F}=\mu-\frac{M_{s}^{2}}{4 \mu} \text {. }
$$

It is now easy to evaluate the difference $\Omega_{\text {unpair. }}-\Omega_{\text {pair. }}$ at the order $M_{s}^{4}$, with the result

$$
\Omega_{\text {pair. }}-\Omega_{\text {unpair. }} \approx \frac{1}{16 \pi^{2}}\left(M_{s}^{4}-4 \Delta^{2} \mu^{2}\right) .
$$

We see that in order to have condensation the condition

$$
\mu>\frac{M_{s}^{2}}{2 \Delta}
$$

must be realized. The problem of one massless and one massive flavor has been studied by Kundu and Rajagopal (2002). One can simulate this situation by letting the two quarks both be massless, but with two different chemical potentials, which is equivalent to having two different Fermi spheres. The big advantage here is that one can use the LOFF analysis discussed in Sec. II.C

Color superconductivity due to the nonvanishing of the condensates of Eqs. (4.1) or (4.3) is the result of a mechanism analogous to the formation of an electron Cooper pair in a BCS superconductor, and, as in BCS superconductivity, the only relevant fermion degrees of freedom are those near the Fermi surface. Therefore a two-dimensional effective-field theory has been developed to describe it. We shall briefly review it below, but our main interest is to delineate another development of color superconductivity, i.e., the presence of a LOFF superconducting phase. In this case, the condensation is generated by the attractive color interaction in the antitriplet channel. This phase of QCD has been mainly studied at low temperatures (see, for example, Alford et al., 2001a, 2001b; Bowers et al., 2001; Leibovich et al., 2001; Rajagopal, 2001; Bowers and Rajagopal, 2002). Like the color-flavor-locked and two-flavor superconducting phases, the QCD LOFF phase can be studied by effective-field theory. ${ }^{4}$ This description is useful for deriving the effective Lagrangian for the Goldstone bosons associated with the breaking of space symmetries, i.e., phonons. It is based on an analogy with the heavy-quark effective-field theory and is called the high-density effective-field theory. To describe these developments we organize this section as follows. In Sec. IV.A we give an outline of the high-density effective-field theory. We specialize the formalism to the color-flavor-locked phase in Sec. IV.B and to the two-color superconducting phase in Sec. IV.C. The final subsections are devoted to the LOFF phase in QCD. In Sec. IV.D, after a general introduction to the subject, we consider a Nambu-JonaLasinio coupling for a QCD liquid formed by quarks

${ }^{4}$ See Casalbuoni, Gatto, Mannerelli, and Nardulli (2001, 2002a); Casalbuoni, Fabiano, et al. (2002); Casalbuoni, Gatto, and Nardulli (2002); Nardulli (2002b). with two flavors. Given the similarities with the BCS four-fermion interaction arising from the electronphonon interactions in metals, we can apply the same formalism discussed in previous sections. In the present case, however, the two species we consider are quarks of different flavors, up and down, with different chemical potentials $\mu_{u}, \mu_{d}$. We limit our analysis to the FuldeFerrell one-plane-wave state. However, the results of Sec. III.C are also valid for the QCD LOFF state. In particular, the guess on the favored structure at $T=0$ discussed by Bowers and Rajagopal (2002) and reviewed in Sec. III.C.4 should point to the cubic structure as the most favored LOFF crystal. In Sec. IV.E, we discuss the differences induced by considering the one-gluon interaction instead of the effective four-fermion interaction. LOFF superconductivity in QCD can be induced not only by a difference in the quark chemical potential but also by mass differences among the quarks. This situation is discussed in Sec. IV.F, which shows the role the strange quark mass can play in favoring the LOFF phase.

\section{A. High-density effective-field theory}

At very high baryonic chemical potential $\mu$ and very low temperature $(T \rightarrow 0)$, it is useful to adopt an effective-field description of QCD known as highdensity effective-field theory (see Beane et al., 2000; Hong, 2000a, 2000b; Casalbuoni, Gatto, and Nardulli, 2001; and, for reviews, Casalbuoni, 2001; Nardulli, 2002a). Let us consider the fermion field

$$
\psi(x)=\int \frac{d^{4} p}{(2 \pi)^{4}} e^{-i p \cdot x} \psi(p) .
$$

Since the relevant degrees of freedom are those near the Fermi surface, we decompose the fermion momentum as

$$
p^{\mu}=\mu v^{\mu}+\ell^{\mu},
$$

where $v^{\mu}=(0, \mathbf{v}), \mathbf{v}$ being the Fermi velocity (for massless fermions $|\mathbf{v}|=1$ ), and $\ell^{\mu}$ is a residual momentum. We also use $V^{\mu}=(1, \mathbf{v}), \widetilde{V}^{\mu}=(1,-\mathbf{v})$.

We now introduce the velocity-dependent, positiveenergy $\psi_{\mathbf{v}}$ and negative-energy $\Psi_{\mathbf{v}}$, left-handed fields via the decomposition

$$
\psi(x)=\int \frac{d \mathbf{v}}{4 \pi} e^{-i \mu v \cdot x}\left[\psi_{\mathbf{v}}(x)+\Psi_{\mathbf{v}}(x)\right] .
$$

Here

$$
\psi_{\mathbf{v}}(x)=e^{i \mu v \cdot x} P_{+} \psi(x)=\int_{|\ell|<\delta} \frac{d^{4} \ell}{(2 \pi)^{4}} e^{-i \ell \cdot x} P_{+} \psi(\ell)
$$

and

$$
\Psi_{\mathbf{v}}(x)=e^{i \mu v \cdot x} P_{-} \psi(x)=\int_{|\ell|<\delta} \frac{d^{4} \ell}{(2 \pi)^{4}} e^{-i \ell \cdot x} P_{-} \psi(\ell) .
$$

$P_{ \pm}$are projectors that for massless quarks are defined by 


$$
P_{ \pm} \equiv P_{ \pm}(\mathbf{v})=\frac{1}{2}(1 \pm \boldsymbol{\alpha} \cdot \mathbf{v}) .
$$

The extension to massive quarks is discussed by Casalbuoni, De Fazio, et al. (2002) and Casalbuoni, Gatto, et al. (2003). The cutoff $\delta$ satisfies $\delta \ll \mu$ while still being much larger than the energy gap.

Using the identities

$$
\begin{aligned}
& \bar{\psi}_{\mathbf{v}} \gamma^{\mu} \psi_{\mathbf{v}}=V^{\mu} \bar{\psi}_{\mathbf{v}} \gamma^{0} \psi_{\mathbf{v}}, \quad \bar{\Psi}_{\mathbf{v}} \gamma^{\mu} \Psi_{\mathbf{v}}=\widetilde{V}^{\mu} \Psi_{\mathbf{v}} \gamma^{0} \Psi_{\mathbf{v}}, \\
& \bar{\psi}_{\mathbf{v}} \gamma^{\mu} \Psi_{\mathbf{v}}=\bar{\psi}_{\mathbf{v}} \gamma_{\perp}^{\mu} \Psi_{\mathbf{v}}, \quad \bar{\Psi}_{\mathbf{v}} \gamma^{\mu} \psi_{\mathbf{v}}=\bar{\Psi}_{\mathbf{v}} \gamma_{\perp}^{\mu} \psi_{\mathbf{v}},
\end{aligned}
$$

and substituting them into the Dirac part of the QCD Lagrangian, we obtain

$$
\begin{aligned}
\mathcal{L}_{D}= & \int \frac{d \mathbf{v}}{4 \pi}\left[\psi_{\mathbf{v}}^{\dagger} i V \cdot D \psi_{\mathbf{v}}+\Psi_{\mathbf{v}}^{\dagger}(2 \mu+i \tilde{V} \cdot D) \Psi_{\mathbf{v}}\right. \\
& \left.+\left(\bar{\psi}_{\mathbf{v}} i D_{\perp} \Psi_{\mathbf{v}}+\text { H.c. }\right)\right] .
\end{aligned}
$$

Here $D_{\perp}=D_{\mu} \gamma_{\perp}^{\mu}$ and $D_{\mu}$ is the covariant derivative, $D^{\mu}=\partial^{\mu}+i g A^{\mu}$. We note that here all of the quark fields are evaluated at the same Fermi velocity. Off-diagonal terms are subleading due to the Riemann-Lebesgue lemma, as they are canceled by the rapid oscillations of the exponential factor in the $\mu \rightarrow \infty$ limit (the Fermi velocity superselection rule). A similar behavior occurs in QCD in the $m_{Q} \rightarrow \infty$ limit, when one uses the heavyquark effective-field theory (see Isgur and Wise, 1989, 1990; Eichten and Hill, 1990; Georgi, 1990, and for reviews, Neubert, 1994; Casalbuoni et al., 1997; Manohar and Wise, 2000).

We can get rid of the negative-energy solutions by integrating out the $\Psi_{\mathbf{v}}$ fields in the generating functional. At tree level this corresponds to solving the equations of motion,

$$
i V \cdot D \psi_{\mathbf{v}}=0
$$

and

$$
\Psi_{\mathbf{v}}=-\frac{i}{2 \mu+i \tilde{V} \cdot D} \gamma_{0} D_{\perp} \psi_{\mathbf{v}},
$$

which shows the decoupling of $\Psi_{\mathrm{v}}$ in the $\mu \rightarrow \infty$ limit. In the resulting effective-field theory for $\psi_{\mathbf{v}}$, only the energy and the momentum parallel to the Fermi velocity are relevant, and the effective-field theory is two dimensional.

It is useful to introduce two separate fields;

$$
\psi_{ \pm} \equiv \psi_{ \pm \mathbf{v}},
$$

so that the average over the Fermi velocities is defined as

$$
\sum_{\mathbf{v}}=\int \frac{d \mathbf{v}}{8 \pi} .
$$

The extra factor of $1 / 2$ occurs here because, after the introduction of the field with opposite velocity $\psi_{-}$, one doubles the degrees of freedom, which implies that the integration is only over half of a solid angle.
In conclusion, if $\mathcal{L}_{0}$ is the free-quark Lagrangian and $\mathcal{L}_{1}$ represents the coupling of quarks to one gluon, the high-density effective Lagrangian can be written as

$$
\mathcal{L}_{D}=\mathcal{L}_{0}+\mathcal{L}_{1}+\mathcal{L}_{2}+(L \rightarrow R),
$$

where

$$
\begin{aligned}
& \mathcal{L}_{0}=\sum_{\mathbf{v}}\left[\psi_{+}^{\dagger} i V \cdot \partial \psi_{+}+\psi_{-}^{\dagger} i \tilde{V} \cdot \partial \psi_{-}\right], \\
& \mathcal{L}_{1}=i g \sum_{\mathbf{v}}\left[\psi_{+}^{\dagger} i V \cdot A \psi_{+}+\psi_{-}^{\dagger} i \widetilde{V} \cdot A \psi_{-}\right],
\end{aligned}
$$

and

$$
\begin{aligned}
\mathcal{L}_{2}= & -\sum_{\mathbf{v}} P^{\mu \nu}\left[\psi_{+}^{\dagger} \frac{1}{2 \mu+i \tilde{V} \cdot D} D_{\mu} D_{\nu} \psi_{+}\right. \\
& \left.+\psi_{-}^{\dagger} \frac{1}{2 \mu+i V \cdot D} D_{\mu} D_{\nu} \psi_{-}\right] .
\end{aligned}
$$

$\mathcal{L}_{2}$ is a nonlocal Lagrangian arising when one integrates over the $\Psi_{\mathbf{v}}$ degrees of freedom in the functional integration. It contains couplings of two quarks to any number of gluons and gives contribution to the gluon Meissner mass. We have put

$$
P^{\mu \nu}=g^{\mu \nu}-\frac{1}{2}\left[V^{\mu} \widetilde{V}^{\nu}+V^{\nu} \widetilde{V}^{\mu}\right] .
$$

This construction is valid for any theory describing massless fermions at high density, provided one excludes degrees of freedom far from the Fermi surface.

\section{B. Color-flavor-locked phase}

Even though we shall consider the LOFF phase for only two flavors, for completeness we present the highdensity effective-field theory for the three-flavor colorflavor-locked phase as well. In the CFL phase the symmetry breaking is induced by the condensates

$$
\left\langle\psi_{i \alpha}^{L T} C \psi_{j \beta}^{L}\right\rangle=-\left\langle\psi_{i \alpha}^{R T} C \psi_{j \beta}^{R}\right\rangle=\frac{\Delta}{2} \epsilon_{\alpha \beta I} \epsilon_{i j I},
$$

where $\psi^{L, R}$ are Weyl fermions and $C=i \sigma_{2}$. Equation (4.27) corresponds to the invariant coupling $\left(\psi \equiv \psi_{L}\right)$ :

$$
-\frac{\Delta}{2} \sum_{I=1,3} \psi^{T} C \epsilon_{I} \psi \epsilon_{I}-(L \rightarrow R)+\text { H.c. },
$$

and $\left(\epsilon_{I}\right)_{a b}=\epsilon_{I a b}$. Neglecting the negative energy components, for the Dirac fermions $\psi_{ \pm}$we introduce the compact notation

$$
\chi=\frac{1}{\sqrt{2}}\left(\begin{array}{c}
\psi_{+} \\
C \psi_{-}^{*}
\end{array}\right)
$$

in a way analogous to Eq. (2.11). We also use a different basis for quark fields:

$$
\psi_{ \pm \mathbf{v}, i \alpha}=\sum_{A=1}^{9} \frac{\left(\lambda_{A}\right)_{i \alpha}}{\sqrt{2}} \psi_{ \pm}^{A} .
$$

The CFL fermionic Lagrangian therefore has the form 


$$
\mathcal{L}_{D}=\mathcal{L}_{0}+\mathcal{L}_{1}+\mathcal{L}_{\Delta}=\sum_{\vec{v}} \sum_{A, B=1}^{9} \chi^{A \dagger}\left(\begin{array}{cc}
i \operatorname{Tr}\left[T_{A} V \cdot D T_{B}\right] & -\Delta_{A B} \\
-\Delta_{A B} & i \operatorname{Tr}\left[T_{A} \widetilde{V} \cdot D^{*} T_{B}\right]
\end{array}\right) \chi^{B}+(L \rightarrow R),
$$

where

$$
\Delta_{A B}=\Delta \operatorname{Tr}\left[\epsilon_{I} T_{A}^{T} \epsilon_{I} T_{B}\right]
$$

and

$$
T_{A}=\frac{\lambda_{A}}{\sqrt{2}} .
$$

Here $\lambda_{9}=\lambda_{0}=\sqrt{\frac{2}{3}} \times \mathbf{1}$. We use the identity

$$
\epsilon_{I} g^{T} \epsilon_{I}=g-\operatorname{Tr} g
$$

where $g$ is any $3 \times 3$ matrix and obtain

$$
\Delta_{A B}=\Delta_{A} \delta_{A B},
$$

where

$$
\Delta_{1}=\cdots=\Delta_{8}=\Delta
$$

and

$$
\Delta_{9}=-2 \Delta .
$$

The CFL free-fermionic Lagrangian therefore assumes the form

$$
\mathcal{L}_{0}+\mathcal{L}_{\Delta}=\sum_{\mathbf{v}} \sum_{A=1}^{9} \chi^{A \dagger}\left(\begin{array}{cc}
i V \cdot \partial & -\Delta_{A} \\
-\Delta_{A} & i \widetilde{V} \cdot \partial
\end{array}\right) \chi^{A}+(L \rightarrow R) .
$$

Clearly the equations of motion following from this Lagrangian are of the same type as the Nambu-Gor'kov equations [see Eq. (2.15)]. For applications of highdensity effective-field theory to the CFL phase we refer the reader to Casalbuoni, Gatto, and Nardulli (2001).

$$
\mathcal{L}_{D}=\mathcal{L}_{0}+\mathcal{L}_{1}+\mathcal{L}_{\Delta}=\sum_{\mathbf{v}} \sum_{A, B=0}^{5} \chi^{A \dagger}\left(\begin{array}{c}
i \operatorname{Tr}\left[\widetilde{T}_{A} V \cdot D \widetilde{T}_{B}\right] \\
-\Delta_{A B}
\end{array}\right.
$$

Here

$$
\begin{aligned}
& \Delta_{A B}=\frac{\Delta}{2} \operatorname{Tr}\left[\tilde{\epsilon} \tilde{\lambda}_{A}^{T} \tilde{\epsilon} \tilde{\lambda}_{B}\right] \quad(A, B=0, \ldots 3), \\
& \Delta_{A B}=0 \quad(A, B=4,5),
\end{aligned}
$$

and

$$
\widetilde{T}_{A}=\frac{\tilde{\lambda}_{A}}{\sqrt{2}} \quad(A=0, \ldots, 5) .
$$

Analogously to Eq. (4.34), we use the identity

\section{Two-flavor superconducting phase}

For the two-flavor case, which encompasses both the superconducting model and the existing calculation in the LOFF phase, we follow a similar approach. The symmetry breaking is induced by the condensates

$$
\left\langle\psi_{i \alpha}^{L T} C \psi_{j \beta}^{L}\right\rangle=-\left\langle\psi_{i \alpha}^{R T} C \psi_{j \beta}^{R}\right\rangle=\frac{\Delta}{2} \epsilon_{\alpha \beta 3} \epsilon_{i j 3},
$$

and the invariant coupling is $\left(\psi \equiv \psi^{L}\right)$

$$
\mathcal{L}_{\Delta}=-\frac{\Delta}{2} \psi^{T} C \epsilon \psi \epsilon-(L \rightarrow R)+\text { H.c. },
$$

where

$$
\epsilon=i \sigma_{2} \text {. }
$$

We use a different basis for the fermion fields by writing the positive-energy effective fields $\psi_{ \pm \mathbf{v}, i \alpha}$ as follows:

$$
\psi_{ \pm \mathbf{v}, i \alpha}=\sum_{A=0}^{5} \frac{\left(\tilde{\lambda}_{A}\right)_{i \alpha}}{\sqrt{2}} \psi_{ \pm}^{A} .
$$

The $\tilde{\lambda}_{A}$ matrices are defined in terms of the usual $\lambda$ matrices as

$$
\begin{aligned}
& \tilde{\lambda_{0}}=\frac{1}{\sqrt{3}} \lambda_{8}+\sqrt{\frac{2}{3}} \lambda_{0}, \quad \tilde{\lambda}_{A}=\lambda_{A} \quad(A=1,2,3), \\
& \tilde{\lambda}_{4}=\frac{\lambda_{4-i 5}}{\sqrt{2}}, \quad \tilde{\lambda_{5}}=\frac{\lambda_{6-i 7}}{\sqrt{2}} .
\end{aligned}
$$

We also define $\tilde{\epsilon}=i \lambda_{2}$. After the introduction, analogously to Eq. (4.29) for the fields $\chi^{A}$, the two-flavor superconducting fermionic Lagrangian assumes the form
$\left.\begin{array}{c}-\Delta_{A B} \\ i \operatorname{Tr}\left[\widetilde{T}_{A} \widetilde{V} \cdot D^{*} \widetilde{T}_{B}\right]\end{array}\right) \chi^{B}+(L \rightarrow R)$.

$$
\widetilde{\boldsymbol{\epsilon}} g^{T} \widetilde{\boldsymbol{\epsilon}}=g-\operatorname{Tr} g ;
$$

we obtain

$$
\Delta_{A B}=\Delta_{A} \delta_{A B},
$$

where $\Delta_{A}$ is defined as

$$
\Delta_{A}=(+\Delta,-\Delta,-\Delta,-\Delta, 0,0) .
$$

Therefore the effective Lagrangian for free quarks in the two-flavor superconducting model can be written 


$$
\mathcal{L}_{0}+\mathcal{L}_{\Delta}=\sum_{\mathbf{v}} \sum_{A=0}^{5} \chi^{A \dagger}\left(\begin{array}{cc}
i V \cdot \partial & -\Delta_{A} \\
-\Delta_{A} & i \widetilde{V} \cdot \partial
\end{array}\right) \chi^{A}+(L \rightarrow R) .
$$

\section{LOFF phase in QCD}

We shall assume here that in the most interesting phenomenological applications, i.e., in compact stars (see Sec. VI.G), there is a significant difference between the Fermi momenta of different flavors. Since this produces a difference in the densities, the BCS phase may be disrupted (Alford, Berges, and Rajagopal, 1999; Schafer and Wilczek, 1999c), and a phase analogous to the LOFF phase might arise. The case of a LOFF phase in QCD was also discussed by Son and Stephanov (2001) and Splittorff et al. (2001) in the context of quark matter at large isospin density. Differences in the Fermi momenta in these examples arise both from differences between chemical potentials, due to the weak equilibrium, and from mass differences between the strange and the up and down quarks. A complete study requires that we take both effects into account. This has been done by Kundu and Rajagopal (2002). We shall discuss this paper below. Here we shall consider a simpler case, in which all the quarks are massless but have different chemical potentials (Alford et al., 2001b). To further simplify the problem, we shall restrict ourselves to the case of two massless quarks with chemical potentials $\mu_{u}$ and $\mu_{d}$ given by

$$
\mu_{u}=\mu+\delta \mu, \quad \mu_{d}=\mu-\delta \mu .
$$

These equations are the same as Eq. (2.2), but now up and down refer to flavor.

Everything goes according to the discussion of Secs. II.B and II.C, except that now the density of the gapped states at the Fermi surface is multiplied by a factor of 4 , which comes from the two colors and the two flavors. In fact, the condensate has the form

$$
\left\langle\psi_{i}^{\alpha} \psi_{j}^{\beta}\right\rangle \propto \epsilon^{\alpha \beta 3} \epsilon_{i j},
$$

where $\alpha, \beta=1,2,3$ and $i, j=1,2$ are, respectively, color and flavor indices. Other differences are in the value of the Fermi velocity, which is $v_{F}=1$, since we are dealing with massless fermions, and in the Fermi momentum, which is given by $p_{F}=\mu$. As a consequence, the density of states is now

$$
\rho=4 \frac{\mu^{2}}{\pi^{2}} .
$$

It follows that the first-order transition from the homogeneous phase to the normal one, in the weak-coupling limit and using Eq. (2.63), is given by

$$
\begin{aligned}
\Omega_{\Delta}(\delta \mu)-\Omega_{0}(\delta \mu) & =\frac{\rho}{4}\left(2 \delta \mu^{2}-\Delta_{0}^{2}\right) \\
& =\frac{\mu^{2}}{\pi^{2}}\left(2 \delta \mu^{2}-\Delta_{0}^{2}\right) \\
& =0 .
\end{aligned}
$$

Applying the results obtained in Sec. II.C to color superconductivity requires some care. For instance, although only two colors are gapped, in order to describe the mixed phase we need a proper treatment of the two ungapped quarks (Bedaque, 2002). Another situation that can be present in QCD but not in condensed matter is the case of equal chemical potentials with different Fermi momenta due to unequal masses. This is discussed by Alford, Berges, and Rajagopal (1990) and Schafer and Wilczek (1999c). However, in the realistic case, different chemical potentials must be considered.

We shall also describe the LOFF phase using the formalism of fields close to the Fermi surface, although in the present case the corrections to leading order are expected to be larger since we are not considering asymptotic values of the chemical potential. This formalism is very close to the Nambu-Gor'kov formalism developed in Sec. II. We consider a four-fermion interaction modeled on a one-gluon exchange, that is,

$$
\mathcal{L}_{I}=-\frac{3}{8} G \bar{\psi} \gamma^{\mu} \lambda^{a} \psi \bar{\psi} \gamma^{\mu} \lambda^{a} \psi
$$

where $\lambda^{a}$ are Gell-Mann matrices. We then introduce the fields $\psi_{i+}^{\alpha}$ through the procedure outlined in Sec. IV.C. We perform the same transformation, $\exp (-i \mu v \cdot x)$, for both flavors. For simplicity, in the rest of this section we shall denote the fields $\psi_{i+}^{\alpha}$ by $\psi_{i}^{\alpha}$. Separating the left-handed and the right-handed modes, the previous interaction can be written as

$$
\begin{aligned}
\mathcal{L}_{I} & =-\frac{G}{2}\left(3 \delta_{\delta}^{\alpha} \delta_{\beta}^{\gamma}-\delta_{\beta}^{\alpha} \delta_{\delta}^{\gamma}\right) \epsilon_{\dot{a} \dot{c}} \epsilon_{b d} \psi_{\alpha \dot{a}}^{\dagger i} \psi_{\gamma \dot{c}}^{\dagger j} \psi_{i b}^{\beta} \psi_{j d}^{\delta} \\
& \equiv V_{\beta \delta}^{\alpha \gamma} \psi_{\alpha}^{\dagger i} \psi_{\gamma}^{\dagger j} \psi_{i}^{\beta} \psi_{j}^{\delta},
\end{aligned}
$$

where, in the last expression, the sum over the spin indices $\dot{a}, \dot{c}, b, d$ is understood and

$$
V_{\beta \delta}^{\alpha \gamma}=-\frac{G}{2}\left(3 \delta_{\delta}^{\alpha} \delta_{\beta}^{\gamma}-\delta_{\beta}^{\alpha} \delta_{\delta}^{\gamma}\right)
$$

In obtaining this result we have used the identities

$$
\sum_{a=1}^{8}\left(\lambda^{a}\right)_{\alpha \beta}\left(\lambda^{a}\right)_{\delta \gamma}=\frac{2}{3}\left(3 \delta_{\alpha \gamma} \delta_{\beta \delta}-\delta_{\alpha \beta} \delta_{\gamma \delta}\right)
$$

and

$$
\left(\sigma_{\mu}\right)_{\dot{a} b}\left(\widetilde{\sigma}^{\mu}\right)_{d \dot{c}}=2 \epsilon_{\dot{a} \dot{c}} \epsilon_{b d} .
$$

Here

$$
\sigma^{\mu}=(1, \boldsymbol{\sigma}), \quad \widetilde{\sigma}^{\mu}=(1,-\boldsymbol{\sigma}),
$$

with $\boldsymbol{\sigma}$ the Pauli matrices. As in Sec. II, we divide $\mathcal{L}_{I}$ into two pieces, 
$\mathcal{L}_{\text {cond }}=V_{\beta \delta}^{\alpha \gamma}\left(\psi_{\alpha}^{\dagger i} \psi_{\gamma}^{\dagger j}\left\langle\psi_{i}^{\beta} \psi_{j}^{\delta}\right\rangle+\psi_{i}^{\beta} \psi_{j}^{\delta}\left\langle\psi_{\alpha}^{\dagger i} \psi_{\gamma}^{\dagger j}\right\rangle\right)+(L \rightarrow R)$

and

$$
\begin{aligned}
\mathcal{L}_{i n t}= & V_{\beta \delta}^{\alpha \gamma}\left(\psi_{\alpha}^{\dagger i} \psi_{\gamma}^{\dagger j}-\left\langle\psi_{\alpha}^{\dagger i} \psi_{\gamma}^{\dagger j}\right\rangle\right)\left(\psi_{i}^{\beta} \psi_{j}^{\delta}-\left\langle\psi_{i}^{\beta} \psi_{j}^{\delta}\right\rangle\right) \\
& +(L \rightarrow R) .
\end{aligned}
$$

The first piece can be written as

$$
\mathcal{L}_{\text {cond }}=-\frac{1}{2} \epsilon_{\alpha \beta 3} \epsilon^{i j}\left(\psi_{i}^{\alpha} \psi_{j}^{\beta} \Delta e^{2 i q \cdot r}+\text { c.c. }\right)+(L \rightarrow R),
$$

where we have defined

$$
\Gamma_{S} e^{2 i \mathbf{q} \cdot \mathbf{r}}=-\frac{1}{2} \epsilon^{\alpha \beta 3} \epsilon_{i j}\left\langle\psi_{\alpha}^{i} \psi_{\beta}^{j}\right\rangle
$$

and

$$
\Delta=G \Gamma_{S} .
$$

The quadratic part of the Lagrangian $\mathcal{L}_{\text {cond }}$ in terms of the Nambu-Gor'kov fields can be written as

$$
\mathcal{L}_{\text {cond }}^{(2)}=\frac{1}{2} \sum_{\alpha, i} \chi_{i}^{\dagger \alpha}\left(S^{-1}\right)_{\alpha j}^{\beta i} \chi_{\beta}^{j},
$$

where, in momentum space,

$$
\left(S^{-1}\right)_{\alpha j}^{\beta i}=\left(\begin{array}{cc}
\delta_{\alpha \beta}\left[\delta_{i j} V \cdot \ell+\delta \mu\left(\sigma_{3}\right)_{i j} \delta\left(\ell-\ell^{\prime}\right)\right] & -\epsilon_{\alpha \beta 3} \epsilon_{i j} \Delta \delta\left(\ell-\ell^{\prime}+2 q\right) \\
-\epsilon_{\alpha \beta 3} \epsilon_{i j} \Delta \delta\left(\ell-\ell^{\prime}-2 q\right) & \delta_{\alpha \beta}\left[\delta_{i j} \tilde{V} \cdot \ell+\delta \mu\left(\sigma_{3}\right)_{i j} \delta\left(\ell-\ell^{\prime}\right)\right]
\end{array}\right),
$$

and $q^{\mu}=(0, \mathbf{q})$. Using this expression and performing the same derivation as in Sec. II, we find the gap equation

$$
\begin{aligned}
\Delta= & 2 i G \Delta \int \frac{d \mathbf{v}}{4 \pi} \frac{d \ell_{0}}{2 \pi} d \ell_{\|} \frac{\mu^{2}}{2 \pi^{2}} \\
& \times \operatorname{Tr} \frac{1}{\left[V \cdot(\ell+q)+\delta \mu \sigma_{3}\right]\left[\tilde{V} \cdot(\ell-q)+\delta \mu \sigma_{3}\right]-\Delta^{2}} .
\end{aligned}
$$

Comparing this result with Eq. (2.39), one notices a factor of 2 coming from the trace on the color; the remaining trace is on the flavor indices where the matrix $\sigma_{3}$ acts. Performing the trace and making explicit the $i \epsilon$ prescriptions for the energy integration, we find

$$
\begin{aligned}
\Delta= & i \frac{G \rho}{2} \Delta \int \frac{d \mathbf{v}}{4 \pi} \int_{-\delta}^{+\delta} d \xi \frac{d E}{2 \pi} \\
& \times \frac{1}{(E-\bar{\mu}+i \epsilon \operatorname{sgn} E)^{2}-\xi^{2}-\Delta^{2}},
\end{aligned}
$$

where $\rho=4 \mu^{2} / \pi^{2}$ is the relevant density at the Fermi surface and we have defined $E=\ell_{0}$ and $\xi=\ell_{\|}$to emphasize the similarity with the original LOFF equation (see Sec. II.C). Moreover,

$$
\bar{\mu}=\delta \mu-\mathbf{v} \cdot \mathbf{q} \text {. }
$$

Performing the integration over the energy we get

$$
1=\frac{G \rho}{2} \int \frac{d \mathbf{v}}{4 \pi} \int_{0}^{\delta} \frac{d \xi}{\sqrt{\xi^{2}+\Delta^{2}}} \theta(\epsilon-|\bar{\mu}|) .
$$

Since

$$
\theta(\epsilon-|\bar{\mu}|)=1-\theta(-\epsilon-\bar{\mu})-\theta(-\epsilon+\bar{\mu}),
$$

we get exactly the LOFF gap equation [compare with Eq. (2.115)], except for the different definition of the density of states.
We have already shown that in the present case there is a first-order transition in $\delta \mu$, between the homogenous state (which from now on will be referred to as the BCS state) and the normal state. Furthermore, from Sec. II.C we know that there is a second-order transition between the LOFF state and the normal one. These results are also valid in the present case with the only change in the density of gapped states at the Fermi surface, which, as already stressed, is now a factor of 4 larger than the one for electrons. We recall from that analysis that around the second-order critical point $\delta \mu_{2}=0.754 \Delta_{0}$ (with $\Delta_{0}$ the BCS gap) we have [see Eq. (3.27)]

$$
\begin{aligned}
\Delta_{L O F F} & =\sqrt{1.757 \delta \mu_{2}\left(\delta \mu_{2}-\delta \mu\right)} \\
& =1.15 \Delta_{0} \sqrt{\frac{\delta \mu_{2}-\delta \mu}{\Delta_{0}}} .
\end{aligned}
$$

As for the grand potential, we have from Eq. (2.63)

$$
\Omega_{B C S}-\Omega_{\text {normal }}=\frac{1}{4} \rho\left(2 \delta \mu^{2}-\Delta_{0}^{2}\right),
$$

and from Eq. (3.28)

$$
\Omega_{L O F F}-\Omega_{\text {normal }}=-0.439 \rho\left(\delta \mu-\delta \mu_{2}\right)^{2} .
$$

These results are summarized in Fig. 14, where we plot the grand potentials for the different phases.

Since the interval $\left(\delta \mu_{1}, \delta \mu_{2}\right)$ is rather narrow, there is practically no difference between the values of $\delta \mu$ corresponding to the BCS-normal transition $\left(\delta \mu_{1}\right.$ $\left.=\Delta_{0} / \sqrt{2}\right)$ and the value corresponding to the BCSLOFF transition. This can be seen easily in Figs. 14 and 15. The figures were obtained by using the previous equations in the Ginzburg-Landau expansion around $\delta \mu_{2}$, but they are a very good approximation to the curves obtained numerically (Takada and Izuyama, 1969; Alford et al., 2000)

All the discussion here has used the weak-coupling limit. For a more correct treatment see Alford et al. 


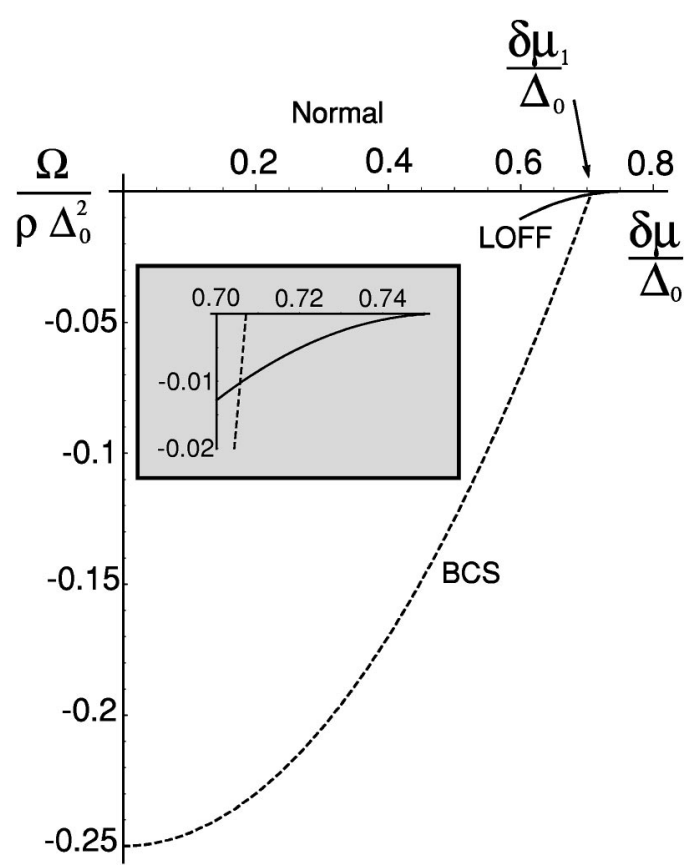

FIG. 14. Differences between the grand potential for the BCS and the normal state and that between the LOFF and the normal state, plotted vs $\delta \mu$. The grand potentials are normalized to $\rho \Delta_{0}^{2}$. The inset shows the intersection of the two curves close to $\delta \mu_{1}$ : solid lines, the LOFF case; dotted lines, the BCS case.

(2000) where the results from the numerical integration of the gap equation are given. In particular, we want to stress the results obtained by these authors for the size of the window. If $\delta \mu_{1}$ and $\delta \mu_{2}$ are evaluated for a general coupling, and at the same time one takes into account corrections from the chemical potential in the measure of integration, the windows get smaller and smaller for increasing BCS gap $\Delta_{0}$. The corrections in the chemical potential arise from the momentum integration, which is made on a shell of height $2 \delta$ but with an integration measure given by $p^{2} d p d \Omega$, rather than $p_{F}^{2} d p d \Omega$ as is usually done in the treatment of the BCS gap in the weak-coupling limit. The results are illustrated in Fig. 16, where the behavior of the critical points vs the BCS gap is shown. The curves are plotted for a range of values of the cutoff $\Lambda$ ranging from 0.8 to

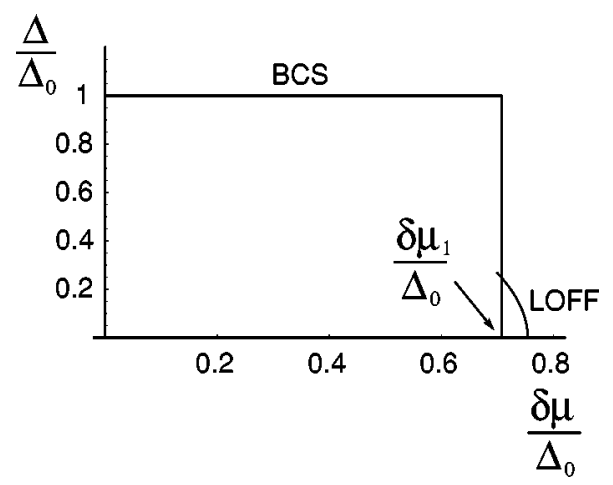

FIG. 15. The condensates of the BCS and LOFF phases vs $\delta \mu$.

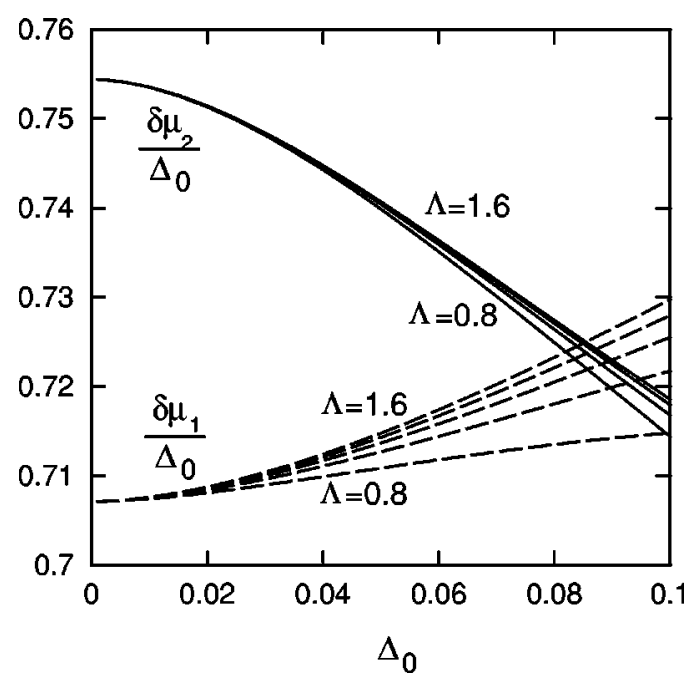

FIG. 16. The critical points $\delta \mu_{1}$ (solid lines) and $\delta \mu_{2}$ (dashed lines), in $\Delta_{0}$ units vs $\Delta_{0}$, for cutoff values $\Lambda=\mu+\delta$ ranging from $0.8 \mathrm{GeV}$ up to $1.6 \mathrm{GeV}$ and for $\mu=0.4 \mathrm{GeV}$. From Alford et al., 2001b.

$1.6 \mathrm{GeV}$. The cutoff dependence is not very strong, in particular, for $\delta \mu_{2}$; moreover, the window closes for $\Delta_{0}$ between 80 and $100 \mathrm{MeV}$, according to the chosen value of $\Lambda$.

Alford et al. (2000) also discuss the presence of a vector condensate in the QCD LOFF phase. The reason why this condensate can be formed in QCD but not in condensed matter is the following: Both in the BCS and in the LOFF phases the coupling is between fermions of the same helicity. In the BCS phase the fermions also have opposite momentum, giving rise to a $J=0$ pair. On the other hand, in the LOFF phase momenta are not aligned exactly. Therefore a small component of $J=1$ condensate may arise. A spin-1 state is symmetric in the spin indices, and therefore Fermi statistics forbids it for electron pairing. On the other hand, in QCD with two flavors one can form a state that is antisymmetric in color and symmetric in flavor, and the Pauli principle is satisfied. Therefore the structure of the vector condensate is (Alford et al., 2000)

$$
\left\langle\left(\sigma_{1}\right)_{i j} \boldsymbol{\epsilon}_{\alpha \beta 3} \psi_{i L}^{\alpha} \sigma^{0 i} \psi_{j L}^{\beta}\right\rangle=-2 i \frac{q^{i}}{|\mathbf{q}|} \Gamma_{V} e^{2 i \mathbf{q} \cdot \mathbf{r}} .
$$

The ratio $\Gamma_{V} / \Gamma_{S}$ is practically constant within the LOFF window, varying between 0.121 at $\delta \mu_{1}$ and 0.133 at $\delta \mu_{2}$. However, this condensate does not contribute to the grand potential in the present case (Alford et al., 2000); therefore it does not change the original LOFF results. The situation is different if, instead of using the NambuJona-Lasino interaction (4.55), one uses the interaction

$$
\begin{aligned}
\mathcal{L}_{I}= & -\frac{3}{8}\left[G_{E}\left(\bar{\psi} \gamma^{0} \lambda^{a} \psi\right)\left(\bar{\psi} \gamma^{0} \lambda^{a} \psi\right)-G_{M}\left(\bar{\psi} \gamma^{i} \lambda^{a} \psi\right)\right. \\
& \left.\times\left(\bar{\psi} \gamma^{i} \lambda^{a} \psi\right)\right] .
\end{aligned}
$$

This expression is not Lorentz invariant, but since we are trying to model QCD at finite density, there is no reason to use a Lorentz-invariant effective action. For 


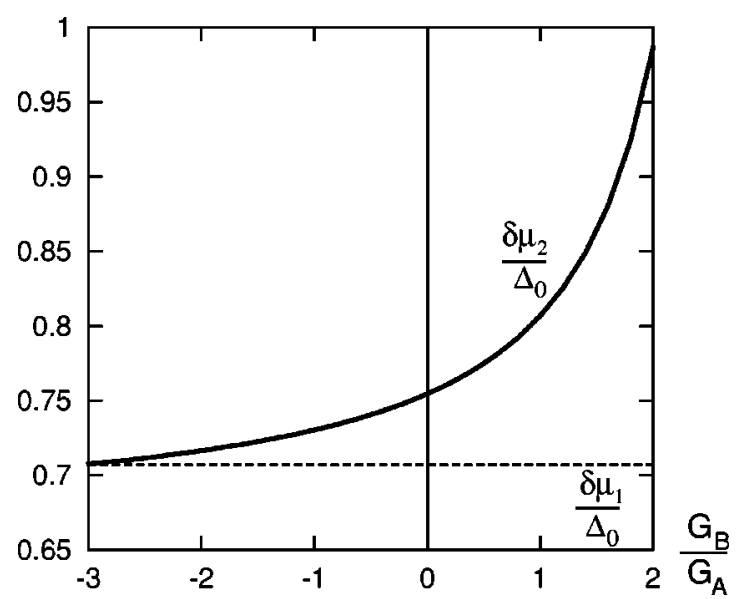

FIG. 17. Variation of the critical points $\delta \mu_{1} / \Delta_{0}$ (dotted line), and $\delta \mu_{2} / \Delta_{0}$ (solid line) with $G_{B} / G_{A}$. From Alford et al., 2001b.

instance, at high density the electric gluons are expected to be screened, whereas the magnetic ones are Landau damped. In particular, it has been shown by Son (1999) that at high density the magnetic gluon exchange dominates the pairing mechanism, which can be simulated assuming $G_{E} \ll G_{M}$.

For the following discussion it is convenient to introduce the quantities

$$
G_{A}=\frac{1}{4}\left(G_{E}+3 G_{M}\right) \text { and } G_{B}=\frac{1}{4}\left(G_{E}-G_{M}\right) .
$$

For $G_{E}=G_{M}=G$, as discussed above one has

$$
G_{A}=G, \quad G_{B}=0 .
$$

At zero density we expect $G_{B}=0$, whereas at high density we expect $G_{E}=0$ or $G_{B} / G_{A}=-1 / 3$. Therefore the relevant physical region for $G_{B} / G_{A}$ should be given by

$$
-\frac{1}{3} \leqslant \frac{G_{B}}{G_{A}} \leqslant 0 .
$$

The gap parameters are now defined by

$$
\Delta=G_{A} \Gamma_{S}, \quad \Delta_{V}=G_{B} \Gamma_{V} .
$$

Since the grand potential and the quasiparticle energy are determined by the gap, for the Lorentz-invariant case, $G_{B}=0$, there is no contribution from the vector condensate. For $G_{B} \neq 0$, one has to solve two coupled gap equations (Alford et al., 2000). The most interesting result found by Alford et al. concerns the LOFF window, which is modified by the presence of the $J=1$ gap. The result is shown in Fig. 17.

The LOFF window closes at $G_{B} / G_{A}=-3$ and increases with increasing $G_{B} / G_{A}$. For $G_{B} / G_{A}$ inside the physical region [see Eq. (4.80)] the maximal opening is for $G_{B}=0$. However, inside the physical region the variation of the window is rather small. Since $\delta \mu_{1}$ is essentially defined by the BCS-normal-state transition, it is given by $\Delta_{0} / \sqrt{2}$, and is independent of the vector condensate. However, the second-order critical point $\delta \mu_{2}$ is rather sensitive to $G_{B} / G_{A}$, the reason being that, for
$G_{A}=0$, the $J=1$ channel is attractive for $G_{B}>0$ and repulsive for $G_{B}<0$. Therefore the stability of the LOFF state is reinforced by the vector condensate in the region $G_{B}>0$.

Let us close this section by considering a different pairing discussed by Deryagin et al. (1992), Park et al. (2000), Shuster and Son (2000), and Rapp et al. (2001). It is a quark-hole pairing with nonzero momentum at large baryon density. This produces a $\langle\bar{q} q\rangle$ condensate varying in space with a wave number $2 \mu$, to be contrasted with $2|\mathbf{q}| \approx 2|\delta \mu|$. This state is energetically favored only for very large values of the number of colors (Deryagin et al., 1992). Park et al. (2000) and Shuster and Son (2000) found that $N_{c}$ should be larger than about 1000 .

\section{E. One-gluon exchange approximation}

The foregoing results were obtained in the case of a Nambu-Jona-Lasinio interaction. The case of the onegluon exchange interaction has been studied by Leibovich et al. (2001). Of course this would be a realistic case only at very high densities (Rajagopal and Shuster, 2000) where, presumably, the CFL phase dominates over the LOFF phase. However, the study of different interactions allows us to understand the model dependence of the LOFF window. Leibovich et al. (2001) used the standard QCD vertex in conjunction with the following propagator for the gluon:

$$
D_{\mu \nu}=\frac{P_{\mu \nu}^{T}}{p^{2}-G(p)}+\frac{P_{\mu \nu}^{L}}{p^{2}-F(p)},
$$

where

$$
\begin{aligned}
& P_{i j}^{T}=\delta_{i j}-\frac{p_{i} p_{j}}{|\mathbf{p}|^{2}}, \quad P_{00}^{T}=P_{0 i}^{T}=0, \\
& P_{\mu \nu}^{L}=-g_{\mu \nu}+\frac{p_{\mu} p_{\nu}}{p^{2}}-P_{\mu \nu}^{T} .
\end{aligned}
$$

Here

$$
G(p)=\frac{\pi}{4} m^{2} \frac{p^{0}}{|\mathbf{p}|}
$$

describes the Landau damping, and

$$
F(p)=m^{2},
$$

where $m^{2}$ is the Meissner mass evaluated for two flavors,

$$
m^{2}=g^{2} \frac{\mu^{2}}{\pi^{2}} \text {. }
$$

The expressions for $F(p)$ and $G(p)$ are obtained in the hard-loop approximation (Le Bellac, 1996) and evaluated here for $p_{0} \ll|\mathbf{p}| \approx \mu$ (we recall that $\mu$ is the average chemical potential). Solving the gap equation, we find that the LOFF window is enlarged by about a factor of 10 at the average chemical potential $\mu=400 \mathrm{MeV}$. In fact, as already noted, whereas $\delta \mu_{1}$ is essentially fixed by the BCS-normal-state transition at the value $\delta \mu_{1}$ 
$=\Delta_{0} / \sqrt{2}, \delta \mu_{2}$ increases dramatically. At $\mu=400 \mathrm{MeV}$, Leibovich et al. (2001) found

$$
\delta \mu_{2}=1.24 \Delta_{0} \Rightarrow \delta \mu_{2}-\delta \mu_{1}=0.55 \Delta_{0},
$$

to be compared with the Nambu-Jona-Lasinio case, in which

$$
\delta \mu_{2}=0.754 \Delta_{0} \Rightarrow \delta \mu_{2}-\delta \mu_{1}=0.05 \Delta_{0} .
$$

At $\mu=10^{3} \mathrm{MeV}$ the window is about 60 times larger than the window for the pointlike case. In general, when $\mu$ is increased, $\delta \mu_{2}$ increases as well. The interpretation of these results, according to Leibovich et al. (2001), goes as follows: For weak coupling, the $q-q$ scattering via one-gluon exchange is mostly in the forward direction. This implies that, after the scattering has taken place, quarks remain close to the angular position they possessed before the scattering, meaning that the theory is essentially $1+1$ dimensional. In fact, in this case the only possible value for $2|\mathbf{q}|$ is $\mu_{d}-\mu_{u}=2 \delta \mu$. This is not the case in $3+1$ dimensions. In fact, as can be seen from Fig. $4,2|\mathbf{q}|$ is generally bigger than $2 \delta \mu$. Furthermore, it is known from the 1+1-dimensional case (Buzdin and Tugushev, 1983; Buzdin and Polonski, 1987) that in the weak-coupling limit $\delta \mu_{2} / \Delta_{0} \rightarrow \infty$. Both these features have been found by Leibovich et al. (2001).

A similar analysis was carried out by Giannakis et al. (2002). The results were somewhat different from those discussed above. In particular, it was found that at weak coupling

$$
\delta \mu_{2}=0.968 \Delta_{0} \Rightarrow \delta \mu_{2}-\delta \mu_{1}=0.26 \Delta_{0},
$$

with an enhancement of the window by a modest factor of 5 with respect to the pointlike interaction. However, the evaluation made in this paper consists of an expansion around the tricritical point (called $\delta_{o n}$ by Giannakis et al.) implying, in particular, an expansion in $|\mathbf{q}|$. In order to compare the results of Giannakis et al. with those of Leibovich and co-workers, one should extrapolate the results of Giannakis et al. (2002) to zero temperature. It is not evident, at least to us, that this can be safely done. Of course, the physical interpretation is also different. According to Giannakis et al. (2002), in $3+1$ dimensions increasing $\delta \mu$ implies a reduction of the phase space and therefore a smaller gap and a smaller $\delta \mu_{2}$. This reduction effect, according to these authors, overcomes the enhancement due to the 1+1-dimensional effect discussed above.

In our opinion the case of the one-gluon exchange in the LOFF phase deserves further study. In fact, a sizable increase in the LOFF window would make the LOFF state very interesting as far as the applications to compact stellar objects are concerned.

\section{F. Mass effects}

Kundu and Rajagopal (2002) studied the combined effect of having two quarks with different chemical potentials when one of the two quarks was massive. In the free case, the Fermi momenta are given (assuming that the pair contains an up and a strange quark) by

$$
p_{F}^{u}=\mu-\delta \mu, \quad p_{F}^{s}=\sqrt{(\mu+\delta \mu)^{2}-M_{s}^{2}} .
$$

Assuming both $\delta \mu / \mu$ and $m_{s} / \mu$ to be much smaller than one, one finds

$$
\left|p_{F}^{u}-p_{F}^{s}\right| \approx 2\left|\delta \mu-\frac{M_{s}^{2}}{4 \mu}\right| .
$$

The effect of $M_{s} \neq 0$ amounts to something more than the simple shift $\delta \mu \rightarrow \delta \mu-M_{s}^{2} / 4 \mu$. In fact, let us recall that at $M_{s}=0$ the BCS condensate is not changed by $\delta \mu$ as long as $\delta \mu<\delta \mu_{1}$. However, $\Delta_{0}$ decreases with $M_{s}$ (see the results by Casalbuoni, De Fazio, et al., 2002 and Kundu and Rajagopal, 2002). Furthermore, for $M_{s}^{2} / \mu^{2}$ $\ll 1$ the decrease is practically linear. This produces corrections to the grand potential of order $\Delta_{0}^{2}(0) M_{s}^{2}$. For small values of $\delta \mu-M_{s}^{2} / 4 \mu$ we have BCS pairing, whereas for large values there is no pairing and the system is in the normal phase. Therefore the BCS-normal transition is $M_{s}$ dependent and occurs for $\delta \mu$ approximately given by

$$
\left|\delta \mu-\frac{M_{s}^{2}}{4 \mu}\right|=\frac{\Delta_{0}\left(M_{s}\right)}{\sqrt{2}} .
$$

It can be noted that, unlike the case $M_{s}=0$, this condition is not symmetric for $\delta \mu \rightarrow-\delta \mu$, and the LOFF phase can exist in two different windows in $\delta \mu$, above $\left(\delta \mu_{2}>\delta \mu_{1}\right)$ and below $\left(\delta \mu_{2}<\delta \mu_{1}\right)$ the BCS region; in any case, for $M_{s}=0$ one gets back the ClogstonChandrasekar limit. In order to discuss the size of the window the correct variable is

$$
\frac{\delta \mu_{2}\left(M_{s}\right)-\delta \mu_{1}\left(M_{s}\right)}{\Delta_{0}\left(M_{s}\right)} .
$$

At weak coupling [small $\Delta_{0}(0)$ ] it is found that the window is essentially the same as for the case $M_{s}=0$. Otherwise the window generally increases with $M_{s}$ as shown in Fig. 18 for various values of $M_{s}$. We have plotted both the cases $\delta \mu>0$ (left panel) and $\delta \mu<0$. This shows that the LOFF phase is rather robust for $M_{s} \neq 0$.

\section{PHONON AND GLUON EFFECTIVE LAGRANGIANS}

Translational and rotational invariance are spontaneously broken within a LOFF phase. The energy gap is not uniform and actually is expected to vary according to some crystalline structure, as a result of the analysis developed in the previous sections. The crystal defined by the space modulation of the gap can fluctuate, and its local deformations define phonon fields $\phi^{(i)}$ that are the Nambu-Goldstone bosons associated with the breaking of translational symmetry. The number of phonon fields is equal to the number of broken generators of the translation group. The existence of long-wavelength oscillations with the phonon dispersion law was already noted by Fulde and Ferrell (1964). More recently an effective Lagrangian for phonons in a QCD medium was developed by Casalbuoni et al. (Casalbuoni, Gatto, et al., 2001, 2002a; Casalbuoni, Fabiano, et al., 2002; Casalbuoni, Gatto, and Nardulli, 2002), and we wish to 

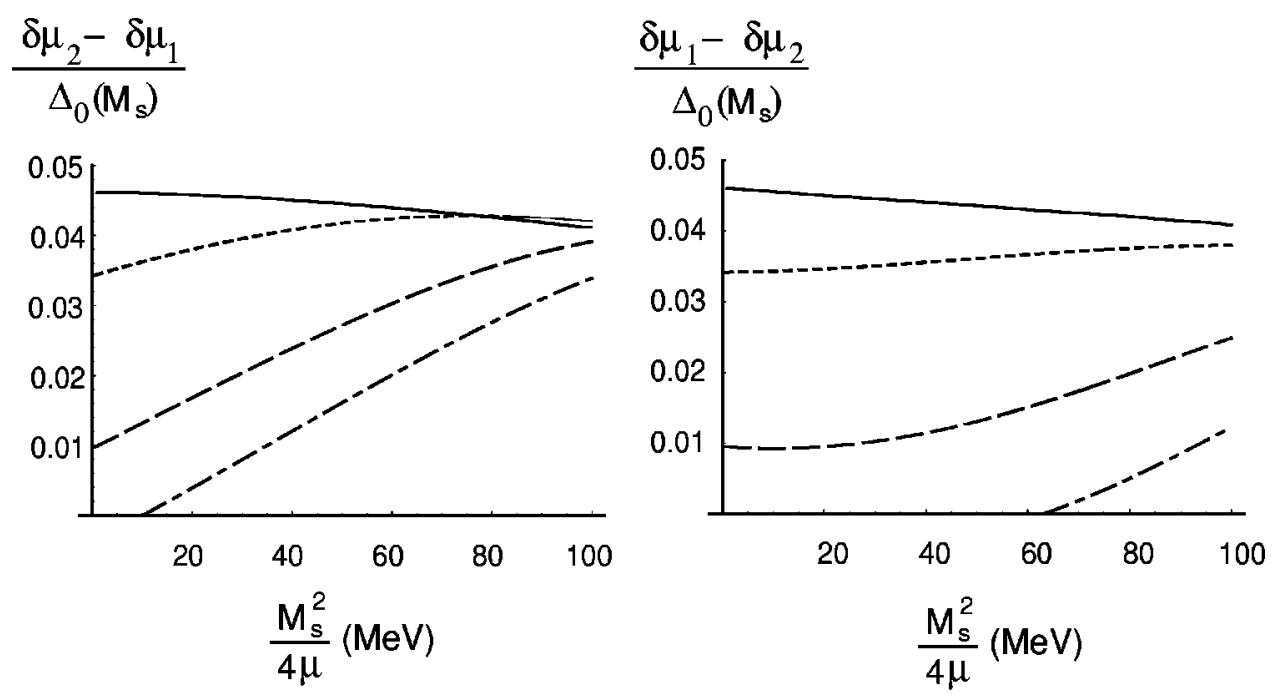

FIG. 18. The width of the LOFF window above and below the BCS region. The four curves correspond to the following values of $\Delta_{0}(0)(\mathrm{MeV})$ : solid line, 10; dotted line, 40; dashed line, 80; dash-dotted line, 100. From Kundu and Rajagopal, 2002.

review it in this section, dedicated predominantly to the QCD LOFF phase. For color superconductivity only the $T \rightarrow 0$ case is physically interesting and we shall consider only this limit. However, the theory developed in this section could be extended to $T \neq 0$ as well as to other physical cases in solid-state or nuclear physics.

Being long-wavelength oscillations of the crystalline LOFF structure, phonons exist only if the quarks of the Cooper pair are in the pairing region. This is a portion of the phase space around the Fermi surface formed by a few annular rings, which are likely to be contiguous, according to Bowers and Rajagopal (2002); see also the discussion in Sec. III.C. The effective-field theory for the phonon fields $\phi^{(i)}$ must display this behavior, and therefore the phonon-quark coupling must vanish outside the pairing region. The mathematical formalization of this behavior is rather involved and some approximation is needed. Casalbuoni, Fabiano, et al. (2002) and Casalbuoni, Gatto, et al. (2002a) write the phonon-quark interaction using the high-density effective-field theory discussed in Sec. IV.A. They introduce effective velocitydependent fermion fields and the Lagrangian as a sum of terms, each characterized by its own Fermi velocity v. Also, the quark-phonon coupling constant becomes velocity dependent and is proportional to

$$
\Delta_{\mathrm{eff}} \propto \Delta \sum_{k} \sum_{\mathbf{v}} \frac{\pi}{R} \delta_{R}\left[h\left(\mathbf{v} \cdot \hat{\mathbf{n}}_{k}\right)\right] .
$$

Here $\hat{\mathbf{n}}_{k}$ are the vectors defining the LOFF crystal, $R$ is a parameter and $\delta_{R}[h(x)]$ is a function that vanishes outside the pairing region. More precisely, it reaches its maximum when the pairing quarks are on the Fermi surface and decreases when they leave it. By this approximation an evaluation of the phonon effective Lagrangian is possible. In Sec. V.A we consider the high-density effective-field theory for the inhomogeneous LOFF state and write the quark-phonon Lagrangian. Below, we discuss two crystalline structures. First we consider the Fulde-Ferrell one-plane-wave structure, which is the benchmark case for the whole LOFF theory. Then we examine the cubic structure, already studied in Sec. III.C.4, because this seems the most favored crystalline structure according to Bowers and Rajagopal (2002). On the basis of symmetry arguments, we can write down the effective phonon Lagrangians for the two cases. This is done in Secs. V.B and V.D, whereas in Secs. V.C and V.E we show how the parameters of the effective Lagrangian can be computed by the high-density effective-field theory. Let us mention here that the parameter $R$ appearing in Eq. (5.1) should be fixed by a comparison of the gap equation computed in the high-density effectivefield theory and the approach discussed in Sec. II.C for the Fulde-Ferrell state and in Sec. III for generic structures. This comparison has not yet been made and therefore, in the discussion below, we leave $R$ as a parameter, even though, in the case of a cubic structure, the requirement that the annular rings be contiguous can be used to fix its value. We conclude this section with a discussion in V.F of the modifications induced by the LOFF pairing of quarks on the gluon Lagrangian.

\section{A. Effective Lagrangian for the LOFF phase}

Let us begin by writing the gap term for the Lagrangian in the presence of an inhomogeneous condensate. As in Sec. II.C, we write the following formula for the LOFF condensate:

$$
\Delta(\mathbf{r})=\sum_{m=1}^{P} \Delta_{m} e^{2 i \mathbf{q}_{m} \cdot \mathbf{r}} .
$$

We shall consider only two cases below:

(a) one plane wave, $P=1$,

(b) cubic structure, $P=8$.

In the former case, we shall take into account the possibility of having both a $J=0$ and a $J=1$ condensate, as discussed above. In the case of the cubic structure, we 
shall consider only a spin-zero condensate, taking $\Delta_{m}$ $\equiv \Delta$, real, $\mathbf{q}_{m}=\hat{\mathbf{n}}_{m} q$ with $\hat{\mathbf{n}}_{m}$ the eight unit vectors defined in Eq. (3.39). To describe the quark condensate in the case of the single plane wave, we consider the Lagrangian term

$$
\begin{aligned}
\mathcal{L}_{\Delta}=\mathcal{L}_{\Delta}^{(s)}+\mathcal{L}_{\Delta}^{(v)}= & -\frac{e^{2 i \mathbf{q} \cdot \mathbf{r}}}{2} \epsilon_{\alpha \beta 3} \psi_{i \alpha}^{T}(x) C\left(\Delta^{(s)} \boldsymbol{\epsilon}_{i j}\right. \\
& \left.+\boldsymbol{\alpha} \cdot \hat{\mathbf{n}} \Delta^{(v)} \sigma_{i j}^{1}\right) \psi_{i \beta}(x)-(L \rightarrow R)+\text { H.c. }
\end{aligned}
$$

which includes both the scalar and the vector condensate.

We introduce velocity-dependent fields as in Eq. (4.13), with factors $\exp \left(i \mu_{i} v_{i} \cdot x\right)$, and we take into account only the positive-energy part, which we write as $\psi_{\mathbf{v}_{\mathbf{i}} ; i \alpha}$ for a quark with flavor $i$ and color $\alpha$; we keep track of the velocities of the two quarks that are not opposite in the LOFF phase. We have

$$
\begin{aligned}
\mathcal{L}_{\Delta}= & -\frac{1}{2} \sum_{\mathbf{v}_{\mathbf{i}}, \mathbf{v}_{\mathbf{j}}} \exp \left\{i \mathbf{r} \cdot \mathbf{f}\left(\mathbf{v}_{\mathbf{i}}, \mathbf{v}_{\mathbf{j}}, \mathbf{q}_{\mathbf{k}}\right)\right\} \boldsymbol{\epsilon}_{\alpha \beta 3} \psi_{-\mathbf{v}_{\mathbf{i}} ; i \alpha}^{T}(x) C\left(\Delta^{(s)} \boldsymbol{\epsilon}_{i j}\right. \\
& \left.+\boldsymbol{\alpha} \cdot \hat{\mathbf{n}} \Delta^{(v)} \sigma_{i j}^{1}\right) \psi_{-\mathbf{v}_{\mathbf{j}} ; \beta}(x)-(L \rightarrow R)+\text { H.c. }
\end{aligned}
$$

where

$$
\mathbf{f}\left(\mathbf{v}_{\mathbf{i}}, \mathbf{v}_{\mathbf{j}}, \mathbf{q}\right)=2 \mathbf{q}-\mu_{i} \mathbf{v}_{\mathbf{i}}-\mu_{j} \mathbf{v}_{\mathbf{j}}
$$

We also define

$$
\mu=\frac{\mu_{1}+\mu_{2}}{2} \text { and } \delta \mu=-\frac{\mu_{1}-\mu_{2}}{2} .
$$

Since $\mathbf{q}=\mathcal{O}\left(\Delta_{2 s c}\right) \ll \mu$, the condition

$$
\mathbf{p}_{1}+\mathbf{p}_{2}=2 \mathbf{q}
$$

in the $\mu \rightarrow \infty$ limit gives

$$
\mathbf{v}_{\mathbf{1}}+\mathbf{v}_{\mathbf{2}}=\mathcal{O}\left(\frac{\delta}{\mu}\right) \text {. }
$$

Taking into account that $P_{+}(-\mathbf{v}) C \alpha^{k} P_{+}(\mathbf{v})$ $=v^{k} P_{+}(-\mathbf{v}) C P_{+}(\mathbf{v})$, we can rewrite Eq. (5.4) as

$$
\begin{aligned}
\mathcal{L}_{\Delta}= & -\frac{1}{2} \sum_{\mathbf{v}_{\mathbf{i}}, \mathbf{v}_{\mathbf{j}}} \exp \left\{i \mathbf{r} \cdot \mathbf{f}\left(\mathbf{v}_{\mathbf{i}}, \mathbf{v}_{\mathbf{j}}, \mathbf{q}_{\mathbf{k}}\right)\right\} \boldsymbol{\epsilon}_{\alpha \beta 3} \psi_{\mathbf{v}_{\mathbf{j}} ; i \alpha}^{T}(x) \\
& \times C\left(\Delta^{(s)} \boldsymbol{\epsilon}_{i j}-\mathbf{v}_{\mathbf{j}} \cdot \hat{\mathbf{n}} \Delta^{(v)} \sigma_{i j}^{1}\right) \psi_{-\mathbf{v}_{\mathbf{j}} ; j \beta}(x) \\
& -(L \rightarrow R)+\text { H.c. }
\end{aligned}
$$

These equations can be easily generalized to the case of a face-centered cube. We shall discuss this generalization below.

\section{B. One-plane-wave structure}

Let us rewrite Eq. (5.9) as follows:

$$
\begin{aligned}
\mathcal{L}_{\Delta}= & -\frac{1}{2} e^{2 i \mathbf{r} \cdot \mathbf{q}} \sum_{\mathbf{v}_{\mathbf{i}}, \mathbf{v}_{\mathbf{j}}} e^{-i\left(\mu_{i} \mathbf{v}_{\mathbf{i}}+\mu_{j} \mathbf{v}_{\mathbf{j}}\right) \cdot \mathbf{r}} \boldsymbol{\epsilon}_{\alpha \beta 3} \psi_{\mathbf{v}_{\mathbf{j}} ; i \alpha}^{T}(x) \\
& \times C\left(\Delta^{(s)} \boldsymbol{\epsilon}_{i j}-\mathbf{v}_{\mathbf{j}} \cdot \hat{\mathbf{n}} \Delta^{(v)} \sigma_{i j}^{1}\right) \psi_{-\mathbf{v}_{\mathbf{j}} ; j \beta}(x) \\
& -(L \rightarrow R)+\text { H.c. }
\end{aligned}
$$

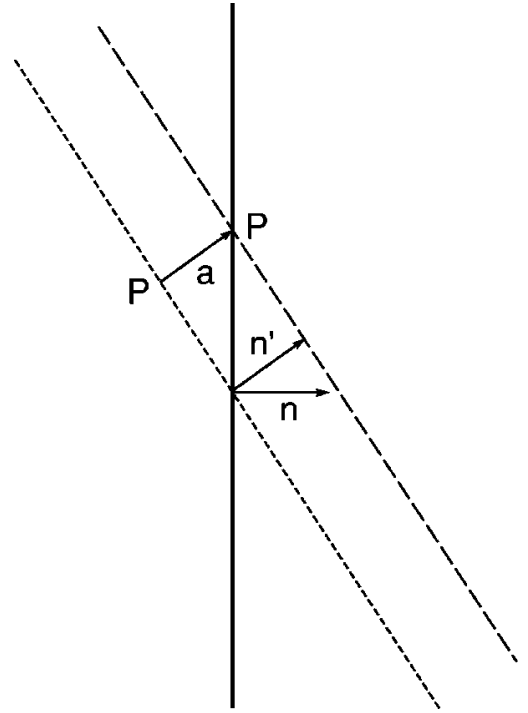

FIG. 19. Effects of rotation and translation at the point $P$. The effect of the rotation $\mathbf{n} \rightarrow \mathbf{n}^{\prime}$ and the effect of the translation $\mathbf{r}$ $\rightarrow \mathbf{r}+\mathbf{a}$ tend to compensate each other.

There are two sources of space-time symmetry breaking in Eq. (5.10), one arising from the exponential term $\exp (2 i \mathbf{r} \cdot \mathbf{q})$, which breaks both translation and rotation invariance, and another in the vector condensate, which breaks rotation invariance. The factor $\exp \left(-i \mu_{i} \mathbf{v}_{\mathbf{i}}\right.$ $-i \mu_{j} \mathbf{v}_{\mathbf{j}}$ ) breaks no space symmetry, since it arises from a field redefinition in a Lagrangian that was originally invariant. For definiteness, let us take the $z$ axis pointing along the direction of q. As a consequence of the breaking of translational invariance along the $z$ axis, Goldstone's theorem predicts the existence of one scalar massless particle, the Nambu-Goldstone boson associated with spontaneous symmetry breaking. The symmetry breaking associated with the vector condensate is not independent of the spontaneous symmetry breaking arising from the exponential term $\exp (2 i \mathbf{r} \cdot \mathbf{q})$, because the direction of $\mathbf{q}$ coincides with the direction $\hat{\mathbf{n}}$ of the vector condensate. For this reason, while there are in general three phonons associated with the breaking of space symmetries, here one Nambu-Goldstone boson is sufficient. The argument is sketched in Fig. 19 and follows from the fact that rotations and translations are not independent transformations, because the result of a translation plus a rotation is locally equivalent to a pure translation.

The Lagrangian (5.10) induces a lattice structure given by parallel planes perpendicular to $\hat{\mathbf{n}}$ :

$$
\hat{\mathbf{n}} \cdot \mathbf{r}=\frac{\pi k}{q} \quad(k=0, \pm 1, \pm 2, \ldots) .
$$

We can give the following physical picture of the lattice structure of the LOFF phase: Due to interaction with the medium, the Majorana masses of the red and green quarks oscillate in the direction $\hat{\mathbf{n}}$, reaching maxima and minima on subsequent planes. The Nambu-Goldstone boson is a long-wavelength, small-amplitude variation of the condensate $\Delta(\mathbf{r})$; formally it is described by the substitution 


$$
\Delta(\mathbf{r})=e^{2 i q \hat{\mathbf{n}} \cdot \mathbf{r}} \Delta \rightarrow e^{i \Phi / f} \Delta,
$$

with

$$
\frac{\Phi}{f}=2 q(\hat{\mathbf{n}}+\delta \mathbf{n}) \cdot(\mathbf{r}+\delta \mathbf{r}) \equiv \frac{\phi}{f}+2 q \hat{\mathbf{n}} \cdot \mathbf{r}
$$

and $\langle\phi\rangle=0$. We assume

$$
\begin{aligned}
& |\hat{\mathbf{n}}+\delta \mathbf{n}|=1, \\
& \langle\delta \mathbf{n}\rangle_{0}=0 .
\end{aligned}
$$

Let us introduce the auxiliary functions $\mathbf{R}$ and $T$,

$$
\mathbf{R}=\hat{\mathbf{n}}+\delta \mathbf{n}, \quad T=2 q \mathbf{R} \cdot \delta \mathbf{r} .
$$

In the second quantization, the lattice fluctuation $\phi$ describes the phonon field. Since it must be small, $T$ and $\mathbf{R}$ are not independent fields and $T$ must depend functionally on $\mathbf{R}$, i.e., $T=F[\mathbf{R}]$, which means

$$
\frac{\Phi}{f}=2 q \mathbf{R} \cdot \mathbf{r}+F[\mathbf{R}] \equiv G[\mathbf{R}, \mathbf{r}] .
$$

The solution of this functional has the form

$$
\mathbf{R}=\mathbf{h}[\Phi]
$$

where $\mathbf{h}$ is a vector built out of the scalar function $\Phi$. By this function one can only ${ }^{5}$ form the vector $\nabla \Phi$; therefore we get

$$
\mathbf{R}=\frac{\boldsymbol{\nabla} \Phi}{|\boldsymbol{\nabla} \Phi|}
$$

which satisfies Eq. (5.14). In terms of the phonon field $\phi$ the vector field $\mathbf{R}$ is given (up to the second order terms in $\phi$ ) by the expression

$$
\begin{aligned}
\mathbf{R}= & \hat{\mathbf{n}}+\frac{1}{2 f q}[\boldsymbol{\nabla} \phi-\hat{\mathbf{n}}(\hat{\mathbf{n}} \cdot \boldsymbol{\nabla} \phi)]+\frac{\hat{\mathbf{n}}}{8 f^{2} q^{2}}\left[3(\hat{\mathbf{n}} \cdot \boldsymbol{\nabla} \phi)^{2}\right. \\
& \left.-|\nabla \phi|^{2}\right]-\frac{\boldsymbol{\nabla} \phi}{4 f^{2} q^{2}}(\hat{\mathbf{n}} \cdot \boldsymbol{\nabla} \phi) .
\end{aligned}
$$

We stress that the only dynamical field is $\phi ; \Phi$ is an auxiliary field with a nonvanishing vacuum expectation value $\langle\Phi\rangle_{0}=2 \mathbf{q} \cdot \mathbf{r}$. As to $\delta \hat{\mathbf{n}}, \mathbf{R}$, and $\delta \mathbf{r}$, they can all be expressed in terms of $\phi$. In conclusion, the interaction term with the Nambu-Goldstone boson field is contained in

$$
\begin{aligned}
\mathcal{L}_{i n t}= & -\frac{1}{2} e^{i \Phi / f} \sum_{\mathbf{v}_{\mathbf{i}}, \mathbf{v}_{\mathbf{j}}} e^{-i\left(\mu_{i} \mathbf{v}_{\mathbf{i}}+\mu_{j} \mathbf{v}_{\mathbf{j}}\right) \cdot \mathbf{r}} \epsilon_{\alpha \beta 3} \psi_{\mathbf{v}_{\mathbf{j}} ; i \alpha}^{T}(x) \\
& \times C\left(\Delta^{(s)} \boldsymbol{\epsilon}_{i j}-\mathbf{v}_{\mathbf{j}} \cdot \mathbf{R} \Delta^{(v)} \sigma_{i j}^{1}\right) \psi_{-\mathbf{v}_{\mathbf{j}} ; j \beta}(x) \\
& -(L \rightarrow R)+\text { H.c. },
\end{aligned}
$$

where the fields $\Phi$ and $\mathbf{R}$ have been introduced in such a

\footnotetext{
${ }^{5}$ In principle there is a second vector, $\mathbf{r}$, on which $\mathbf{R}$ could depend linearly, but this possibility is excluded because $\mathbf{R}$ is a vector field transforming under translations as $\mathbf{R}(\mathbf{r}) \rightarrow \mathbf{R}^{\prime}\left(\mathbf{r}^{\prime}\right)$ $=\mathbf{R}(\mathbf{r})$.
}

way to reproduce Eq. (5.10) in the ground state. At first order in the fields, one gets the following trilinear coupling:

$$
\begin{aligned}
\mathcal{L}_{\phi \psi \psi}= & -\frac{i \phi}{2 f} \sum_{\mathbf{v}_{\mathbf{i}}, \mathbf{v}_{\mathbf{j}}} e^{i \mathbf{r} \cdot \mathbf{f}\left(\mathbf{v}_{\mathbf{i}}, \mathbf{v}_{\mathbf{j}}, \mathbf{q}\right)}\left[\Delta^{(s)} \boldsymbol{\epsilon}_{i j}\right. \\
& \left.-\mathbf{v}_{\mathbf{j}} \cdot \hat{\mathbf{n}} \Delta^{(v)} \sigma_{i j}^{1}\right] \epsilon_{\alpha \beta 3} \psi_{\mathbf{v}_{\mathbf{j}} ; i \alpha}^{T} C \psi_{-\mathbf{v}_{\mathbf{j}} ; j \beta} \\
& -\frac{1}{4 f q} \sum_{\mathbf{v}_{\mathbf{i}}, \mathbf{v}_{\mathbf{j}}} e^{i \mathbf{r} \cdot \mathbf{f}\left(\mathbf{v}_{\mathbf{i}}, \mathbf{v}_{\mathbf{j}}, \mathbf{q}\right)}\left(-\mathbf{v}_{\mathbf{j}}\right) \cdot[\boldsymbol{\nabla} \phi \\
& -\hat{\mathbf{n}}(\hat{\mathbf{n}} \cdot \boldsymbol{\nabla} \phi)] \Delta^{(v)} \sigma_{i j}^{1} \boldsymbol{\epsilon}_{\alpha \beta 3} \psi_{\mathbf{v}_{\mathbf{j}} ; i \alpha}^{T} C \psi_{-\mathbf{v}_{\mathbf{j}} ; j \beta} \\
& -(L \rightarrow R)+\text { H.c. }
\end{aligned}
$$

We also write down the quadrilinear coupling:

$$
\begin{aligned}
\mathcal{L}_{\phi \phi \psi \psi}= & \frac{\phi^{2}}{4 f^{2}} \sum_{\mathbf{v}_{\mathbf{i}}, \mathbf{v}_{\mathbf{j}}} e^{i \mathbf{r} \cdot \mathbf{f}\left(\mathbf{v}_{\mathbf{i}}, \mathbf{v}_{\mathbf{j}}, \mathbf{q}\right)}\left[\Delta^{(s)} \epsilon_{i j}\right. \\
& \left.-\mathbf{v}_{\mathbf{j}} \cdot \hat{\mathbf{n}} \Delta^{(v)} \sigma_{i j}^{1}\right] \epsilon_{\alpha \beta 3} \psi_{\mathbf{v}_{\mathbf{j}} ; i \alpha}^{T} C \psi_{-\mathbf{v}_{\mathbf{j}} ; j \beta} \\
& -\frac{i \phi}{2 f} \sum_{\mathbf{v}_{\mathbf{i}}, \mathbf{v}_{\mathbf{j}}} e^{i \mathbf{r} \cdot \mathbf{f}\left(\mathbf{v}_{\mathbf{i}}, \mathbf{v}_{\mathbf{j}}, \mathbf{q}\right)}\left(-\mathbf{v}_{\mathbf{j}}\right) \cdot[\boldsymbol{\nabla} \phi \\
& -\hat{\mathbf{n}}(\hat{\mathbf{n}} \cdot \boldsymbol{\nabla} \phi)] \Delta^{(v)} \sigma_{i j}^{1} \epsilon_{\alpha \beta 3} \psi_{\mathbf{v}_{j} ; i \alpha}^{T} C \psi_{-\mathbf{v}_{\mathbf{j}} ; j \beta} \\
& -\frac{1}{8 f^{2} q^{2}} \sum_{\mathbf{v}_{\mathbf{i}}, \mathbf{v}_{\mathbf{j}}} e^{i \mathbf{r} \cdot \mathbf{f}\left(\mathbf{v}_{\mathbf{i}}, \mathbf{v}_{\mathbf{j}}, \mathbf{q}\right)}\left[-\frac{\mathbf{v}_{\mathbf{j}} \cdot \hat{\mathbf{n}}}{2}\left[3(\hat{\mathbf{n}} \cdot \boldsymbol{\nabla} \phi)^{2}\right.\right. \\
& \left.\left.-|\boldsymbol{\nabla} \phi|^{2}\right]+\left(\mathbf{v}_{\mathbf{j}} \cdot \boldsymbol{\nabla} \phi\right)(\hat{\mathbf{n}} \cdot \boldsymbol{\nabla} \phi)\right] \\
& \times \Delta^{(v)} \sigma_{i j}^{1} \epsilon^{\alpha \beta 3} \psi_{\mathbf{v}_{\mathbf{j}} ; i \alpha}^{T} C \psi_{-\mathbf{v}_{\mathbf{j}} ; j \beta}-(L \rightarrow R)+\text { H.c. }
\end{aligned}
$$

Through a bosonization procedure, one can derive an effective Lagrangian for the Nambu-Goldstone boson field. This will be done below. For the moment we derive the general properties of the phonon effective Lagrangian. It must contain only derivative terms. Polynomial terms are forbidden by translation invariance, since $\phi$ is not an invariant field. In order to write the kinetic terms, it is better to use the auxiliary field $\Phi$, which behaves as a scalar under both rotations and translation. To avoid the presence of polynomial terms in the phonon Lagrangian, one has to exclude polynomial terms in the auxiliary field $\Phi$ as well; therefore the Lagrangian should be constructed only with derivative terms. The most general invariant Lagrangian will contain a tower of space-derivative terms (Casalbuoni, Gatto, et al., 2001). In fact, since $\langle\boldsymbol{\nabla} \Phi\rangle=2 \mathbf{q}$ is not a small quantity, we cannot limit the expansion in the spatial derivatives of $\Phi$ to any finite order. Therefore we write

$$
\mathcal{L}\left(\phi, \partial_{\mu} \phi\right)=\frac{f^{2}}{2}\left[\dot{\Phi}^{2}-\sum_{n=1}^{\infty} c_{n}\left(|\nabla \Phi|^{2}\right)^{n}\right] .
$$

In this Lagrangian, $\Phi$ must be thought of as a function of the phonon field $\phi$, since 


$$
|\nabla \Phi|^{2}=4 q^{2}+\frac{4 q}{f} \hat{\mathbf{n}} \cdot \boldsymbol{\nabla} \phi+\frac{1}{f^{2}}|\boldsymbol{\nabla} \phi|^{2},
$$

with similar expression for higher powers. At the lowest order in the derivatives of the phonon field $\phi$ we get, neglecting a constant term,

$\mathcal{L}\left(\phi, \partial_{\mu} \phi\right)=\frac{1}{2}\left[\dot{\phi}^{2}-v_{\|}^{2}\left|\nabla_{\|} \phi\right|^{2}-v^{2}\left(4 q f \nabla_{\|} \phi+|\nabla \phi|^{2}\right)\right]$,

where $\boldsymbol{\nabla}_{\|} \phi=\hat{\mathbf{n}} \cdot \boldsymbol{\nabla} \phi$, and $v_{\|}^{2}, v^{2}$ are constants.

\section{Parameters of the phonon effective Lagrangian: one plane wave}

In order to derive the parameters of the phonon Lagrangian (5.26) it is useful to make an approximation. We assume that $\delta \mu \sim \Delta_{2 s c} \ll \delta \ll \mu$. Clearly, we cannot simply take the $\mu \rightarrow \infty$ limit in the exponential term $\exp \left\{i \mathbf{r} \cdot \mathbf{f}\left(\mathbf{v}_{\mathbf{i}}, \mathbf{v}_{\mathbf{j}}, \mathbf{q}_{\mathbf{k}}\right)\right\}$ in Eq. (5.9); therefore we consider a smeared amplitude as follows:

$$
\begin{aligned}
& \lim _{\mu \rightarrow \infty} \exp \left\{i \mathbf{r} \cdot \mathbf{f}\left(\mathbf{v}_{\mathbf{i}}, \mathbf{v}_{\mathbf{j}}, \mathbf{q}_{\mathbf{k}}\right)\right\} \\
& \quad \equiv \lim _{\mu \rightarrow \infty} \int d \mathbf{r}^{\prime} \exp \left\{i \mathbf{r}^{\prime} \cdot \mathbf{f}\left(\mathbf{v}_{\mathbf{i}}, \mathbf{v}_{\mathbf{j}}, \mathbf{q}_{\mathbf{k}}\right)\right\} g\left(\mathbf{r}, \mathbf{r}^{\prime}\right) .
\end{aligned}
$$

We assume a smearing function

$$
g\left(\mathbf{r}, \mathbf{r}^{\prime}\right)=g\left(\mathbf{r}-\mathbf{r}^{\prime}\right)=\prod_{k=1}^{3} \frac{\sin \left[\frac{\pi q\left(r_{k}-r_{k}^{\prime}\right)}{R}\right]}{\pi\left(r_{k}-r_{k}^{\prime}\right)}
$$

and we evaluate Eq. (5.27) in the $\mu \rightarrow \infty$ limit by taking $\mathbf{q}$ along the $z$ axis, and using the identity

$$
\int d^{3} \mathbf{r}^{\prime} \exp \left\{i \mathbf{r}^{\prime} \cdot \mathbf{f}\right\} g\left(\mathbf{r}-\mathbf{r}^{\prime}\right)=\exp \{i \mathbf{r} \cdot \mathbf{f}\}\left(\frac{\pi}{R}\right)^{3} \delta_{R}^{3}\left(\frac{\mathbf{f}}{2 q}\right),
$$

where

$$
\delta_{R}(x)=\left\{\begin{array}{cc}
\frac{R}{\pi} & \text { for }|x|<\frac{\pi}{2 R} \\
0 & \text { elsewhere. }
\end{array}\right.
$$

For the components $x$ and $y$ of $\mathbf{f}$ we get

$$
\left|\left(\mu_{1} v_{1}+\mu_{2} v_{2}\right)_{x, y}\right|<\frac{\pi q}{R},
$$

i.e., approximately (for $\delta \mu \ll \mu$ )

$$
\left|\left(v_{1}+v_{2}\right)_{x, y}\right|<\frac{\pi q}{R \mu} .
$$

From this, in the high-density limit, it follows that

$$
\mathbf{v}_{\mathbf{1}}=-\mathbf{v}_{\mathbf{2}}+\mathcal{O}(\delta \mu / \mu) \text {. }
$$

We have already used this result in Eq. (4.17) in connection with the Riemann-Lebesgue lemma and in Eq. (5.8). A more accurate result is as follows. If $\theta_{1}$ and $\theta_{2}$ are the angles of $\mathbf{v}_{\mathbf{1}}$ and $\mathbf{v}_{\mathbf{2}}$ with respect to the $z$ axis one gets

$$
\theta_{1}=\theta_{2}+\pi+\frac{2 \delta \mu}{\mu} \tan \theta_{2} .
$$

For the $z$ component we get

$$
f_{z}=2 q h\left(\cos \theta_{2}\right),
$$

where

$$
h(x)=1+\frac{x \mu_{2}}{2 q}\left(-1+\sqrt{1-\frac{4 \mu \delta \mu}{\mu_{2}^{2} x^{2}}}\right),
$$

and, neglecting corrections of order $\delta \mu / \mu$,

$$
h(x)=1-\frac{\cos \theta_{q}}{x}, \quad \cos \theta_{q}=\frac{\delta \mu}{q} .
$$

Notice that $\theta_{q}=\psi_{0} / 2$ where $\psi_{0}$ is the angle depicted in Fig. 4; see also Eq. (2.117). The two factors $\pi / R$ arising from the $x$ and $y$ components are absorbed into a wavefunction renormalization of the quark fields, in both the kinetic and the gap terms. As for the $z$ component, one remains with the factor

$$
\frac{\pi}{R} e^{i 2 q h z} \delta_{R}[h(\mathbf{v} \cdot \hat{\mathbf{n}})] \approx \frac{\pi}{R} \delta_{R}[h(\mathbf{v} \cdot \hat{\mathbf{n}})]
$$

in the gap term, whereas for the kinetic term one gets a factor of 1. We have assumed $\exp [i 2 q h z]=1$ in Eq. (5.38) owing to the presence of the $\delta_{R}$ function, which, in the $R / \pi \rightarrow \infty$ limit, enhances the domain of integration where $h=0$. We shall discuss this approximation below.

Equation (5.30) defines a region where $\delta_{R} \neq 0$, i.e., a domain where pairing between the two quarks can occur; it corresponds to the pairing region in the analysis of Fulde and Ferrell (1964) and Bowers et al. (2001), in contrast with the blocking region, where $\delta_{R}=0$. The pairing region intersects the Fermi surface with a "ring" whose size depends on the value of $R$. As we noted above, $R=\infty$ implies the vanishing of the pairing region, and therefore one expects $R \rightarrow \infty$ at the second-order phase transition (Casalbuoni, Fabiano, et al., 2002). The precise value of $R$ should be fixed by the gap equation. Since this calculation has not yet been made, for the purpose of this paper we leave $R$ as a parameter.

In conclusion we can approximate Eq. (5.9) as follows:

$$
\begin{aligned}
\mathcal{L}_{\Delta}= & -\frac{1}{2} \sum_{\mathbf{v}} \frac{\pi}{R} \delta_{R}[h(\mathbf{v} \cdot \hat{\mathbf{n}})] \epsilon_{\alpha \beta 3} \psi_{\mathbf{v} ; i \alpha}^{T}(x) C\left(\Delta^{(s)} \epsilon_{i j}\right. \\
& \left.-\mathbf{v} \cdot \hat{\mathbf{n}} \Delta^{(v)} \sigma_{i j}^{1}\right) \psi_{-\mathbf{v} ; j \beta}(x)-(L \rightarrow R)+\text { H.c. } \quad
\end{aligned}
$$

Using the same notation as in Sec. IV.C, we can write the effective Lagrangian as

$$
\begin{aligned}
\mathcal{L}_{0}+\mathcal{L}_{1}+\mathcal{L}_{\Delta}= & \sum_{\mathbf{v}} \sum_{A, B=0}^{5} \chi^{A \dagger} \\
& \times\left(\begin{array}{cc}
i \operatorname{Tr}\left[\widetilde{T}_{A}^{\dagger} V \cdot D \widetilde{T}_{B}\right] & -\Delta_{A B}^{\dagger} \\
-\Delta_{A B} & i \operatorname{Tr}\left[\widetilde{T}_{A}^{\dagger} \widetilde{V} \cdot D^{*} \widetilde{T}_{B}\right]
\end{array}\right) \\
& \times \chi^{B}+(L \rightarrow R) .
\end{aligned}
$$

Here 


$$
\chi^{A}=\frac{1}{\sqrt{2}}\left(\begin{array}{c}
\psi_{+}^{A} \\
C \psi_{-}^{A *}
\end{array}\right)
$$

and

$$
\widetilde{T}_{A}=\frac{\widetilde{\lambda}_{A}}{\sqrt{2}} \quad(A=0, \ldots, 5) .
$$

The matrix $\Delta_{A B}$ vanishes for $A$ or $B=4$ or 5 , while, for $A, B=0, \ldots, 3$, it is given by

$$
\Delta_{A B}=\left(\Delta_{\mathrm{eff}}^{(s)} \tau_{A B}-\mathbf{v} \cdot \hat{\mathbf{n}} \Delta_{\mathrm{eff}}^{(v)} \sigma_{A B}\right),
$$

with

$$
\begin{aligned}
\tau_{A B} & =\left(\begin{array}{cccc}
1 & 0 & 0 & 0 \\
0 & -1 & 0 & 0 \\
0 & 0 & -1 & 0 \\
0 & 0 & 0 & -1
\end{array}\right), \\
\sigma_{A B} & =\left(\begin{array}{cccc}
0 & 0 & 0 & -1 \\
0 & 0 & -i & 0 \\
0 & +i & 0 & 0 \\
+1 & 0 & 0 & 0
\end{array}\right),
\end{aligned}
$$

and

$$
\begin{aligned}
& \Delta_{\mathrm{eff}}^{(s)}=\frac{\Delta^{(s)} \pi}{R} \delta_{R}[h(\mathbf{v} \cdot \hat{\mathbf{n}})], \\
& \Delta_{\mathrm{eff}}^{(v)}=\frac{\Delta^{(v)} \pi}{R} \delta_{R}[h(\mathbf{v} \cdot \hat{\mathbf{n}})] .
\end{aligned}
$$

In the present approximation the quark propagator is given by

$$
\begin{aligned}
D_{A B}\left(\ell, \ell^{\prime \prime}\right)= & (2 \pi)^{4} \delta^{4}\left(\ell-\ell^{\prime \prime}\right) \\
& \times \sum_{C}\left(\begin{array}{ll}
\frac{\widetilde{V} \cdot \ell \delta_{A C}}{\widetilde{D}_{C B}(\ell)} & \frac{\Delta_{A C}^{\dagger}}{D_{C B}(\ell)} \\
\frac{\Delta_{A C}}{\widetilde{D}_{C B}(\ell)} & \frac{V \cdot \ell \delta_{A C}}{D_{C B}(\ell)}
\end{array}\right),
\end{aligned}
$$

where

$$
\begin{aligned}
& D_{C B}(\ell)=\left(V \cdot \ell \tilde{V} \cdot \ell-\Delta \Delta^{\dagger}\right)_{C B}, \\
& \widetilde{D}_{C B}(\ell)=\left(V \cdot \ell \widetilde{V} \cdot \ell-\Delta^{\dagger} \Delta\right)_{C B} .
\end{aligned}
$$

On the other hand, the propagator for the fields $\chi^{4,5}$ does not contain gap mass terms and is given by

$$
D\left(\ell, \ell^{\prime}\right)=(2 \pi)^{4} \delta^{4}\left(\ell-\ell^{\prime}\right)\left(\begin{array}{cc}
(V \cdot \ell)^{-1} & 0 \\
0 & (\tilde{V} \cdot \ell)^{-1}
\end{array}\right) .
$$

For the other fields $\chi^{A}, A=0, \ldots, 3$, it is useful to go to a representation in which $\Delta \Delta^{\dagger}$ and $\Delta^{\dagger} \Delta$ are diagonal. This is accomplished by performing a unitary transformation which transforms the basis $\chi^{A}$ into a new basis $\tilde{\chi}^{A} \mathrm{de}$ fined by

$$
\tilde{\chi}^{A}=R_{A B} \chi^{B},
$$

with

$$
R_{A B}=\frac{1}{\sqrt{2}}\left(\begin{array}{cccc}
1 & 0 & 0 & 1 \\
0 & 1 & -i & 0 \\
0 & +i & -1 & 0 \\
1 & 0 & 0 & -1
\end{array}\right)
$$

In the new basis we have

$$
\begin{aligned}
& \left(\Delta^{\dagger}\right)_{A B}=\alpha_{A} \delta_{A B}, \\
& \left(\Delta^{\dagger} \Delta\right)_{A B}=\widetilde{\alpha}_{A} \delta_{A B},
\end{aligned}
$$

where

$$
\begin{aligned}
& \alpha_{0}=\alpha_{2}=\widetilde{\alpha}_{2}=\widetilde{\alpha}_{3}=\left(\Delta_{\text {eff }}^{(s)}-\mathbf{v} \cdot \hat{\mathbf{n}} \Delta_{\text {eff }}^{(v)}\right)^{2}, \\
& \alpha_{1}=\alpha_{3}=\widetilde{\alpha}_{0}=\widetilde{\alpha}_{1}=\left(\Delta_{\text {eff }}^{(s)}+\mathbf{v} \cdot \hat{\mathbf{n}} \Delta_{\text {eff }}^{(v)}\right)^{2} .
\end{aligned}
$$

For further reference we also define

$$
\begin{aligned}
\mu_{C}= & \left(\Delta_{\mathrm{eff}}^{(s)}-\mathbf{v} \cdot \hat{\mathbf{n}} \Delta_{\mathrm{eff}}^{(v)}, \Delta_{\mathrm{eff}}^{(s)}+\mathbf{v} \cdot \hat{\mathbf{n}} \Delta_{\mathrm{eff}}^{(v)}, \Delta_{\mathrm{eff}}^{(s)}-\mathbf{v} \cdot \hat{\mathbf{n}} \Delta_{\mathrm{eff}}^{(v)}\right. \\
& \left.\Delta_{\mathrm{eff}}^{(s)}+\mathbf{v} \cdot \hat{\mathbf{n}} \Delta_{\mathrm{eff}}^{(v)}\right)
\end{aligned}
$$

In the basis $\tilde{\chi}$ the three-point and four-point couplings of Eqs. (5.22) and (5.23) are written

$$
\mathcal{L}_{3}+\mathcal{L}_{4}=\sum_{\mathbf{v}} \sum_{A=0}^{3} \tilde{\chi}^{A \dagger}\left(\begin{array}{cc}
0 & -g_{3}^{\dagger}-g_{4}^{\dagger} \\
-g_{3}-g_{4} & 0
\end{array}\right) \tilde{\chi}^{B} .
$$

Here

$$
\begin{aligned}
& g_{3}=\left[\frac{i \phi \Delta_{\mathrm{eff}}^{(s)}}{f} \tau_{A B}+\hat{O}[\phi] \sigma_{A B}\right], \\
& g_{4}=\left[-\frac{\phi^{2} \Delta_{\mathrm{eff}}^{(s)}}{2 f^{2}} \tau_{A B}+\left(\frac{i \phi}{f} \hat{O}[\phi]+\hat{Q}[\phi]\right) \sigma_{A B}\right],
\end{aligned}
$$

with

$$
\begin{aligned}
\hat{O}[\phi]= & \frac{1}{2 f q} \mathbf{v} \cdot[\boldsymbol{\nabla} \phi-\hat{\mathbf{n}}(\hat{\mathbf{n}} \cdot \boldsymbol{\nabla} \phi)] \Delta_{\mathrm{eff}}^{(v)}, \\
\hat{Q}[\phi]= & \frac{\Delta_{\mathrm{eff}}^{(v)}}{4 f^{2} q^{2}}\left[\frac{\mathbf{v} \cdot \hat{\mathbf{n}}}{2}\left[3(\hat{\mathbf{n}} \cdot \boldsymbol{\nabla} \phi)^{2}-|\boldsymbol{\nabla} \phi|^{2}\right]-(\mathbf{v} \cdot \boldsymbol{\nabla} \phi)\right. \\
& \times(\hat{\mathbf{n}} \cdot \boldsymbol{\nabla} \phi)] .
\end{aligned}
$$

Terms in $g_{3}$ and $g_{4}$ that are proportional to $\tau_{A B}$ arise from the expansion of exp $i \phi / f$ alone, whereas terms proportional to $\sigma_{A B}$ also get contributions from the expansion of $\mathbf{R}$ in the vector condensate. The effective action for the Nambu-Goldstone boson is obtained at the lowest order as is shown by the diagrams in Fig. 20. The result of the calculation of the two diagrams at second order in the momentum expansion is 

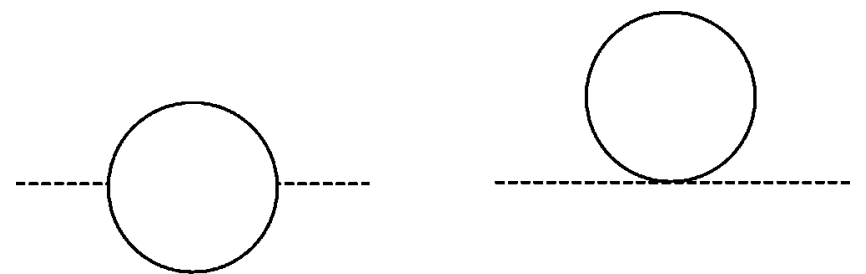

FIG. 20. Diagrams of the Nambu-Goldstone boson: (a) Selfenergy; (b) tadpole.

$$
\begin{aligned}
\Pi(p)_{s . e .}= & \frac{i \mu^{2}}{16 \pi^{3} f^{2}} \sum_{\mathbf{v}} \sum_{C=0}^{3} \int d^{2} \ell\left[\frac{4 \alpha_{C}^{2}}{D_{C}^{2}(\ell)}\right. \\
& -\frac{4 \alpha_{C} V \cdot \ell \tilde{V} \cdot \ell}{D_{C}^{2}(\ell)}-4 \alpha_{C}^{2} \frac{V \cdot p \tilde{V} \cdot p}{D_{C}^{3}(\ell)} \\
& \left.-\left(\frac{\Delta_{\mathrm{eff}}^{(v)}}{q}\right)^{2} \omega^{2}(\vec{p})\left(\frac{2 \alpha_{C}}{D_{C}^{2}(\ell)}+\frac{1}{D_{C}(\ell)}\right)\right] \\
\Pi(p)_{\text {tad }}= & \frac{i \mu^{2}}{16 \pi^{3} f^{2}} \sum_{\mathbf{v}} \sum_{C=0}^{3} \int \frac{d^{2} \ell}{D_{C}(\ell)}\left[4 \alpha_{C}-\frac{\Delta_{\mathrm{eff}}^{(v)}}{q^{2}} \mu_{C}\right. \\
& \left.\times\left(-p_{x}^{2}-p_{y}^{2}+2 p_{z}^{2}-2 \mathbf{p} \cdot \mathbf{v} p_{z}\right)\right],
\end{aligned}
$$

where

$$
D_{C}(\ell)=\ell_{0}^{2}-\ell_{\|}^{2}-\alpha_{C}+i \epsilon,
$$

with $\mu_{c}$ defined in Eq. (5.53) and

$$
\omega(\mathbf{p})=\mathbf{p} \cdot \mathbf{v}-(\mathbf{p} \cdot \hat{\mathbf{n}})(\mathbf{v} \cdot \hat{\mathbf{n}}) .
$$

To perform the calculation we shall take the limit $R$ $\rightarrow \infty$, when the $\delta_{R}$ function becomes the Dirac delta. We handle the $\delta_{R}$ functions by using Fermi's "golden rule"; in the numerator, in the presence of a product of two $\delta_{R}$, we replace one $\delta_{R}$ function with the Dirac delta and for the other one we take

$$
\frac{\pi \delta_{R}[h(x)]}{R} \rightarrow \frac{\pi \delta_{R}(0)}{R} \rightarrow 1 .
$$

A similar substitution is performed in the denominator. Moreover, we use

$$
\int \frac{d^{2} \ell}{\left[D_{C}(\ell)\right]^{3}}=-\frac{i \pi}{2 \alpha_{C}^{2}} .
$$

Therefore we have

$$
\begin{aligned}
\frac{\pi}{R} \delta_{R}[h(\mathbf{v} \cdot \hat{\mathbf{n}})] \frac{\pi}{R} \delta_{R}[h(\mathbf{v} \cdot \hat{\mathbf{n}})] & \rightarrow \frac{\pi}{R} \delta[h(\mathbf{v} \cdot \hat{\mathbf{n}})] \\
& =\frac{\pi}{R} \delta\left[1-\frac{\delta \mu}{q \mathbf{v} \cdot \hat{\mathbf{n}}}\right] \\
& =k_{R} \delta\left[\mathbf{v} \cdot \hat{\mathbf{n}}-\frac{\delta \mu}{q}\right],
\end{aligned}
$$

with

$$
k_{R}=\frac{\pi|\delta \mu|}{q R} .
$$

At second order in the momentum expansion we get

$\Pi(p)=-\frac{\mu^{2} k_{R}}{2 \pi^{2} f^{2}} \sum_{\mathbf{v}} \delta\left[\mathbf{v} \cdot \hat{\mathbf{n}}-\frac{\delta \mu}{q}\right]\left[V_{\mu} \widetilde{V}_{\nu} p^{\mu} p^{\nu}+\Omega^{(v)}(\vec{p})\right]$.

Here

$$
\begin{aligned}
\Omega^{(v)}(\mathbf{p})= & -\left(\frac{\Delta^{(v)}}{q}\right)^{2} \omega^{2}(\mathbf{p})\left(2-\frac{1}{2} \sum_{C=0}^{3} \operatorname{arcsinh} \frac{\delta}{\left|\mu_{c}\right|}\right) \\
& +\frac{\Delta^{(v)}}{2 q^{2}} \Phi(\mathbf{p}) \sum_{C=0}^{3} \mu_{C} \times \operatorname{arcsinh} \frac{\delta}{\left|\mu_{c}\right|} \\
\approx & -2\left(\frac{\Delta^{(v)}}{q}\right)^{2}\left(1-\ln \frac{2 \delta}{\Delta^{(s)}}\right)\left[\omega^{2}(\mathbf{p})+\mathbf{v} \cdot \hat{\mathbf{n}} \Phi(\mathbf{p})\right],
\end{aligned}
$$

where we have used the result (Alford et al., 2001b) $\Delta^{(v)} \ll \Delta^{(s)}$ and

$$
\Phi(\mathbf{p})=\left(3 p_{z}^{2}-\mathbf{p}^{2}\right) \mathbf{v} \cdot \hat{\mathbf{n}}-2 \mathbf{p} \cdot \mathbf{v} p_{z} .
$$

From

$$
\mathcal{L}_{\text {eff }}(p)=-\frac{\mu^{2} k_{R}}{2 \pi^{2} f^{2}} \sum_{\mathbf{v}} \delta\left\{\mathbf{v} \cdot \hat{\mathbf{n}}-\frac{\delta \mu}{q}\right\} V_{\mu} \widetilde{V}_{\nu} p_{\mu} \phi p_{\nu} \phi,
$$

after averaging over the Fermi velocities, we obtain

$$
\mathcal{L}_{\text {eff }}=\frac{1}{2}\left[\left(\dot{\phi}_{k}\right)^{2}-v_{\perp}^{2}\left(\partial_{x} \phi_{k}\right)^{2}-v_{\perp}^{2}\left(\partial_{y} \phi_{k}\right)^{2}-v_{\|}^{2}\left(\partial_{z} \phi_{k}\right)^{2}\right] .
$$

We obtain canonical normalization for the kinetic term provided

$$
f^{2}=\frac{\mu^{2} k_{R}}{4 \pi^{2}}
$$

On the other hand,

$$
\begin{aligned}
& v_{\perp}^{2}=\frac{1}{2} \sin ^{2} \theta_{q}+\left(1-3 \cos ^{2} \theta_{q}\right)\left(1-\log \frac{2 \delta}{\Delta_{0}}\right)\left(\frac{\Delta^{(v)}}{q}\right)^{2}, \\
& v_{\|}^{2}=\cos ^{2} \theta_{q} .
\end{aligned}
$$

In conclusion, we get the anisotropic phonon dispersion law:

$$
E(\vec{p})=\sqrt{v_{\perp}^{2}\left(p_{x}^{2}+p_{y}^{2}\right)+v_{\|}^{2} p_{z}^{2}} .
$$

Besides the anisotropy related to $v_{\perp} \neq v_{\|}$, there is another source of anisotropy, due to the fact that $p_{z}$, the component of the momentum perpendicular to the planes (5.11), unlike $p_{x}$ and $p_{y}$, is a quasimomentum and not a real momentum. The difference can be better appreciated in coordinate space, where the effective Lagrangian reads

$$
\begin{aligned}
\mathcal{L}= & \frac{1}{2}\left[\left(\dot{\phi}_{k}\right)^{2}-v_{\perp}^{2}\left(\partial_{x} \phi_{k}\right)^{2}\right. \\
& \left.-v_{\perp}^{2}\left(\partial_{y} \phi_{k}\right)^{2}-v_{\|}^{2}\left(\frac{q}{\pi}\right)^{2}\left(\phi_{k}-\phi_{k-1}\right)^{2}\right] .
\end{aligned}
$$




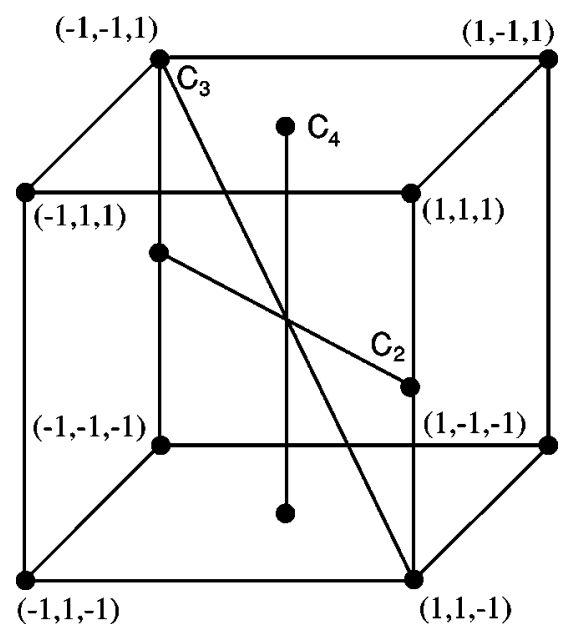

FIG. 21. Symmetry axes $C_{2}, C_{3}$, and $C_{4}$ of the cube.

The effective action for the field $\phi, S[\phi]$, is obtained by the Lagrangian as follows:

$$
S=\int d t d x d y \frac{\pi}{q} \sum_{k=-\infty}^{+\infty} \mathcal{L}(\phi(t, x, y, k \pi / q))
$$

In the action, bilinear terms of the type $\phi_{k} \phi_{k^{\prime}}$ with $k$ $\neq k^{\prime}$ may arise. These terms correspond in the continuum limit to derivatives with respect to the $z$ direction. However, in the long-distance limit $\ell \gg \pi / q$, the set of fields $\phi_{k}(x, y)$ becomes a function $\phi(x, y, z)$ and the last term in Eq. (5.73) can be approximated by $v_{\|}^{2}\left(\partial_{z} \phi\right)^{2}$.

\section{Cubic structure}

The space dependence of the condensate corresponding to a cubic lattice is

$$
\Delta(\mathbf{r})=\Delta \sum_{k=1}^{8} \exp \left\{2 i q \hat{\mathbf{n}}_{k} \cdot \mathbf{r}\right\}
$$

where the eight unit vectors $\hat{n}_{k}$ are given in Eq. (3.39) and

$$
q=\pi / a \text {. }
$$

In Fig. 21 some of the symmetry axes of this cube are shown: they are denoted as $C_{4}$ (the three fourfold axes), $C_{3}$ (the four threefold axes), and $C_{2}$ (the six twofold axes).

To describe the quark condensate, we add a term $\mathcal{L}_{\Delta}$ completely analogous to Eq. (5.10). By the same procedure used for the plane-wave condensate we have

$$
\begin{aligned}
& \mathcal{L}_{\Delta}=-\frac{\Delta}{2} \sum_{k=1}^{8} \sum_{\mathbf{v}} \frac{\pi}{R} \delta_{R}\left[h\left(\mathbf{v} \cdot \hat{\mathbf{n}}_{k}\right)\right] \epsilon_{i j} \epsilon_{\alpha \beta 3} \psi_{\mathbf{v} ; i \alpha}^{T}(x) \\
& \times C \psi_{-\mathbf{v} ; j \beta}(x)-(L \rightarrow R)+\text { H.c. } \\
& \mathcal{L}_{0}+\mathcal{L}_{1}+\mathcal{L}_{\Delta} \text { is still given by Eq. (5.40) but now } \\
& \Delta_{\text {eff }}=\frac{\Delta \pi}{R} \sum_{k=1}^{8} \delta_{R}\left[h\left(\mathbf{v} \cdot \hat{\mathbf{n}}_{k}\right)\right]
\end{aligned}
$$

the quark propagator is given by Eq. (5.46) with $\Delta_{\text {eff }}$ given by Eq. (5.78).

An interesting point should be noted. This equation shows that the pairing region for the cubic LOFF condensate is formed by eight distinct rings, each associated with one vertex of the cube and having as its symmetry axis one of the threefold axes $C_{3}$. According to the analysis of Bowers and Rajagopal (2002), the LOFF vacuum state corresponds to a situation in which these domains have at most one common point. Given the symmetry of the cubic structure, we can limit the analysis to one pair of rings, for example, those associated with the vertices $\mathbf{n}_{1}, \mathbf{n}_{5}$. The common point between these two rings lies on the $C_{2}$ axis and has $\mathbf{v}$ $=1 / \sqrt{2}(1,1,0)$. Since it must also belong to the boundary of the two pairing regions, we have the condition

$$
\left|h\left(\mathbf{v} \cdot \hat{\mathbf{n}}_{1}\right)\right|=\frac{\pi}{2 R},
$$

which implies

$$
R=\frac{\pi}{2 h(\sqrt{2 / 3})} .
$$

Using Eq. (5.36) one gets

$$
R \approx 18 \text {. }
$$

The condensate (5.75) breaks both translations and rotations. It is, however, invariant under the discrete group $O_{h}$, the symmetry group of the cube. This can be seen by noticing that the condensate is invariant under the following coordinate transformations:

$$
\begin{array}{ll}
R_{1}: & x_{1} \rightarrow x_{1}, \quad x_{2} \rightarrow x_{3}, \quad x_{3} \rightarrow-x_{2}, \\
R_{2}: \quad x_{1} \rightarrow-x_{3}, \quad x_{2} \rightarrow x_{2}, & x_{3} \rightarrow x_{1}, \\
R_{3}: \quad x_{1} \rightarrow x_{2}, \quad x_{2} \rightarrow-x_{1}, & x_{3} \rightarrow x_{3}, \\
I: \quad x_{1} \rightarrow-x_{1}, \quad x_{2} \rightarrow-x_{2}, & x_{3} \rightarrow-x_{3},
\end{array}
$$

that is, rotations of $\pi / 2$ around the coordinate axes, and inversion with respect to the origin. Since the group $O_{h}$ is generated by the previous four elements, the invariance follows at once.

The crystal defined by the condensate (5.75) can fluctuate, and its local deformations define three phonon fields $\phi^{(i)}$ that are the Nambu-Goldstone bosons associated with the breaking of translational symmetry. They can be formally introduced following the same procedure discussed for the single-plane-wave case. One effects the substitution in Eq. (5.75)

$$
2 q x^{i} \rightarrow \frac{\Phi^{(i)}(x)}{f}=\frac{2 \pi}{a} x^{i}+\frac{\phi^{(i)}(x)}{f},
$$

where the three auxiliary scalar fields $\Phi^{(i)}$ satisfy

$$
\left\langle\frac{\Phi^{(i)}}{f}\right\rangle_{0}=\frac{2 \pi}{a} x_{i},
$$

whereas for the phonon fields one has

$$
\left\langle\phi^{(i)}(x)\right\rangle_{0}=0 .
$$


One therefore has three fluctuating fields $\phi_{k_{1} k_{2} k_{3}}^{(i)}$ for any elementary cube defined by discrete coordinates

$$
x_{k_{1}}=\frac{k_{1} \pi}{q}, \quad y_{k_{2}}=\frac{k_{2} \pi}{q}, \quad z_{k_{3}}=\frac{k_{3} \pi}{q},
$$

i.e.,

$$
\phi_{k_{1} k_{2} k_{3}}^{(i)} \equiv \phi^{(i)}\left(t, x_{k_{1}}, y_{k_{2}}, z_{k_{3}}\right) .
$$

The interaction term with the Nambu-Goldstone boson fields is therefore given by an equation similar to Eq. (5.74):

$$
\begin{aligned}
S_{i n t}= & -\int d t\left(\frac{\pi}{q}\right)^{3} \sum_{k_{1}, k_{2}, k_{3}=-\infty}^{+\infty} \sum_{\mathbf{v}} \sum_{m=1}^{8} \Delta \\
& \times \exp \left\{i \varphi_{k_{1} k_{2} k_{3}}^{(m)} / f\right\} \epsilon_{i j} \epsilon_{\alpha \beta 3} \psi_{\mathbf{v} ; i \alpha}^{T} C \psi_{-\mathbf{v} ; j \beta} \\
& -(L \rightarrow R)+\text { H.c., }
\end{aligned}
$$

where

$$
\varphi_{k_{1} k_{2} k_{3}}^{(m)}=\sum_{i=1}^{3} \epsilon_{i}^{(m)} \phi_{k_{1} k_{2} k_{3}}^{(i)}
$$

and the eight vectors $\boldsymbol{\epsilon}^{(m)}$ are given by

$$
\left(\boldsymbol{\epsilon}_{i}^{(m)}\right) \equiv \sqrt{3} \hat{\mathbf{n}}_{m} .
$$

The complete effective action for the Nambu-Goldstone boson fields $\phi^{(i)}$ will be of the form

$$
\begin{aligned}
S= & \int d t\left(\frac{\pi}{q}\right)^{3} \\
& \times \sum_{k_{1}, k_{2}, k_{3}=-\infty}^{+\infty} \mathcal{L}\left(\phi^{(i)}\left(t, k_{1} \pi / q, k_{2} \pi / q, k_{3} \pi / q\right)\right) .
\end{aligned}
$$

In the low-energy limit, i.e., for wavelengths much longer than the lattice spacing $\sim 1 / q$, the fields $\phi_{k_{1}, k_{2}, k_{3}}^{(i)}$ vary almost continuously and can be imagined as continuous functions of the three space variables $x, y$, and $z$.

The coupling of the quark fields to the NambuGoldstone boson fields generated by the condensate can be written as

$$
\Delta \psi^{T} C \psi \sum_{\epsilon_{i}= \pm} \exp \left\{i\left(\epsilon_{1} \Phi^{(1)}+\epsilon_{2} \Phi^{(2)}+\epsilon_{3} \Phi^{(3)}\right)\right\},
$$

making the theory invariant under translations and rotations. These symmetries are broken spontaneously in the vacuum defined by Eq. (5.84). In order to write down the effective Lagrangian for the phonon fields $\phi^{(i)}$, it is useful to start with the effective Lagrangian for the auxiliary fields $\Phi^{(i)}$ which must have rotational and translational invariance and $O_{h}$ symmetry on the fields $\Phi^{(i)}$. The last requirement follows from the invariance of the coupling (5.92) under the group $O_{h}$ acting upon $\Phi^{(i)}$. The phonon fields $\phi^{(i)}(x)$ and the coordinates $x^{i}$ must transform under the diagonal discrete group obtained from the direct product of the rotation group acting over the coordinates and the $O_{h}$ group acting over
$\Phi^{(i)}(x)$. This is indeed the symmetry left after the breaking of translational and rotational invariance. The most general low-energy effective Lagrangian displaying these symmetries is

$$
\begin{aligned}
\mathcal{L}= & \frac{f^{2}}{2} \sum_{i=1,2,3}\left(\dot{\Phi}^{(i)}\right)^{2} \\
& +\mathcal{L}_{\mathrm{s}}\left(I_{2}\left(\nabla \Phi^{(i)}\right), I_{4}\left(\nabla \Phi^{(i)}\right), I_{6}\left(\nabla \Phi^{(i)}\right)\right),
\end{aligned}
$$

where

$$
\begin{aligned}
& I_{2}\left(X_{i}\right)=X_{1}^{2}+X_{2}^{2}+X_{3}^{2}, \\
& I_{4}\left(X_{i}\right)=X_{1}^{2} X_{2}^{2}+X_{2}^{2} X_{3}^{2}+X_{3}^{2} X_{1}^{2}, \\
& I_{6}\left(X_{i}\right)=X_{1}^{2} X_{2}^{2} X_{3}^{2}
\end{aligned}
$$

are the three basic symmetric functions of three variables. At lowest order in the fields $\phi^{(i)}$, and at second order in the derivatives, one gets (Casalbuoni, Gatto, and Nardulli, 2002)

$$
\begin{aligned}
\mathcal{L}= & \frac{1}{2} \sum_{i=1,2,3}\left(\dot{\phi}^{(i)}\right)^{2}-\frac{a}{2} \sum_{i=1,2,3}\left|\nabla \phi^{(i)}\right|^{2} \\
& -\frac{b}{2} \sum_{i=1,2,3}\left(\partial_{i} \phi^{(i)}\right)^{2}-c \sum_{i<j=1,2,3} \partial_{i} \phi^{(i)} \partial_{j} \phi^{(j)},
\end{aligned}
$$

which depends on three arbitrary parameters.

\section{E. Parameters of the phonon effective Lagrangian: cubic crystal}

The parameters $a, b$, and $c$ appearing in Eq. (5.95) are computed by a method similar to that used in Sec. V.C. One puts

$$
\varphi^{(m)}(t, \vec{r})=\sum_{i=1}^{3} \epsilon_{i}^{(m)} \phi^{(i)}(t, \vec{r}),
$$

which allows one to write the three-point and four-point couplings as follows:

$$
\mathcal{L}_{3}+\mathcal{L}_{4}=\sum_{\bar{v}} \sum_{A=0}^{3} \tilde{\chi}^{A \dagger}\left(\begin{array}{cc}
0 & -g_{3}^{\dagger}-g_{4}^{\dagger} \\
-g_{3}-g_{4} & 0
\end{array}\right) \tilde{\chi}^{B} .
$$

Here

$$
\begin{aligned}
& g_{3}=\sum_{m=1}^{8} \frac{\pi \Delta}{R} \delta_{R}\left[h\left(\mathbf{v} \cdot \hat{\mathbf{n}}_{m}\right)\right] \frac{i \varphi^{(m)}}{f} \tau_{A B}, \\
& g_{4}=-\sum_{m=1}^{8} \frac{\pi \Delta}{R} \delta_{R}\left[h\left(\mathbf{v} \cdot \hat{\mathbf{n}}_{m}\right)\right] \frac{\left(\varphi^{(m)}\right)^{2}}{2 f^{2}} \tau_{A B},
\end{aligned}
$$

to be compared with Eqs. (5.55) and (5.56), which are valid for the one-plane-wave form of the condensate (we have here neglected the vector condensate). To perform the calculation, one employs the propagator given in Eq. (5.46) with $\Delta_{\text {eff }}$ given in Eq. (5.78) and the interaction vertices in Eq. (5.97). The result of the calculation of the two diagrams in Fig. 20 at second order in the momentum expansion is 


$$
\begin{aligned}
\mathcal{L}_{\text {eff }}(p)_{s . e .}= & i \frac{4 \times 4 \mu^{2}}{16 \pi^{3} f^{2}} \sum_{\mathbf{v}} \sum_{m, k=1}^{8} \frac{1}{2}\left(\frac{\pi \Delta}{R}\right)^{2} \delta_{R}\left[h\left(\mathbf{v} \cdot \hat{\mathbf{n}}_{m}\right)\right] \\
& \times\left(i \varphi^{(m)}\right) \delta_{R}\left[h\left(\mathbf{v} \cdot \hat{\mathbf{n}}_{k}\right)\right]\left(i \varphi^{(k)}\right) \\
& \times \int \frac{d^{2} \ell}{D(\ell) D(\ell+p)} \\
\times & -2 \Delta_{\mathrm{eff}}^{2}+V \cdot \ell \widetilde{V} \cdot(\ell+p) \\
+ & \tilde{V} \cdot \ell V \cdot(\ell+p)] \\
\mathcal{L}_{\mathrm{eff}}(p)_{\text {tad }}= & i \frac{4 \times 4 \mu^{2}}{16 \pi^{3} f^{2}} \sum_{\mathbf{v}} \sum_{m=1}^{8} \int \frac{d^{2} \ell}{D(\ell)} \frac{\pi \Delta \Delta_{\mathrm{eff}}}{R} \\
& \times \delta_{R}\left[h\left(\mathbf{v} \cdot \hat{\mathbf{n}}_{m}\right)\right]\left(\varphi^{(m)}\right)^{2}
\end{aligned}
$$

where

$$
D(\ell)=\ell_{0}^{2}-\ell_{\|}^{2}-\Delta_{\text {eff }}^{2}+i \epsilon,
$$

and, analogous to Eq. (5.45),

$$
\Delta_{\text {eff }}=\frac{\Delta \pi}{R} \sum_{k=1}^{8} \delta_{R}\left[h\left(\mathbf{v} \cdot \hat{\mathbf{n}}_{k}\right)\right] .
$$

From Eqs. (5.99) and (5.101), one can easily check that the Goldstone theorem is satisfied and the phonons are massless. As a matter of fact one has

$$
\begin{aligned}
\mathcal{L}_{\text {mass }}= & \mathcal{L}_{\text {eff }}(0)_{\text {s.e. }}+\mathcal{L}_{\text {eff }}(0)_{\text {tad }} \\
= & i \frac{4 \times 4 \mu^{2}}{16 \pi^{3} f^{2}} \frac{\pi \Delta}{R} \sum_{\mathbf{v}} \int \frac{d^{2} \ell}{D(\ell)} \\
& \times\left[-\sum_{m, k=1}^{8} \frac{\pi \Delta}{R} \delta_{R}\left[h\left(\mathbf{v} \cdot \hat{\mathbf{n}}_{m}\right)\right]\right. \\
& \times \varphi^{(m)} \delta_{R}\left[h\left(\mathbf{v} \cdot \hat{\mathbf{n}}_{k}\right)\right] \varphi^{(k)} \\
& \left.+\Delta_{\text {eff }} \sum_{m=1}^{8} \delta_{R}\left[h\left(\mathbf{v} \cdot \hat{\mathbf{n}}_{m}\right)\right]\left(\varphi^{(m)}\right)^{2}\right] .
\end{aligned}
$$

In the double sum of the rhs of Eq. (5.103), only the terms with $m=k$ survive and one immediately verifies the validity of Goldstone's theorem, i.e., the vanishing of Eq. (5.103). Note that in this approximation the masses of the Goldstone bosons vanish because the pairing regions are not overlapping, signaling that when they do overlap it is not at the minimum of the free energy (see Bowers and Rajagopal, 2002).

At second order in the momentum expansion one has

$$
\begin{aligned}
\mathcal{L}_{\text {eff }}(p)= & i \frac{4 \times 4 \mu^{2}}{16 \pi^{3} f^{2}} \sum_{\mathbf{v}} \sum_{m, k=1}^{8} \frac{1}{2}\left(\frac{\pi \Delta}{R}\right)^{2} \delta_{R}\left[h\left(\mathbf{v} \cdot \hat{\mathbf{n}}_{m}\right)\right] \\
& \times\left(i \varphi^{(m)}\right) \delta_{R}\left[h\left(\mathbf{v} \cdot \hat{\mathbf{n}}_{k}\right)\right]\left(i \varphi^{(k)}\right) \\
& \times \int d^{2} \ell \frac{2 \Delta_{\mathrm{eff}}^{2} V \cdot p \widetilde{V} \cdot p}{[D(\ell)]^{3}} .
\end{aligned}
$$

Using the result

$$
\int \frac{d^{2} \ell}{[D(\ell)]^{3}}=-\frac{i \pi}{2 \Delta_{\mathrm{eff}}^{4}},
$$

and the absence of off-diagonal terms in the double sum, one obtains the effective Lagrangian in the form

$$
\begin{aligned}
\mathcal{L}_{\text {eff }}(p)= & -\frac{\mu^{2}}{2 \pi^{2} f^{2}} \sum_{\mathbf{v}}\left(\frac{\pi \Delta}{R}\right)^{2} \sum_{k=1}^{8} \frac{\left\{\delta_{R}\left[h\left(\mathbf{v} \cdot \mathbf{n}_{k}\right)\right]\right\}^{2}}{\Delta_{\text {eff }}^{2}} \\
& \times(V \cdot p) \varphi^{(k)}(\tilde{V} \cdot p) \varphi^{(k)}
\end{aligned}
$$

To perform this calculation one can exploit the large value found for $R$ and use the same approximations as in Sec. V.C. The sum over $k$ in Eq. (5.106) gives

$$
\begin{aligned}
& \sum_{k=1}^{8} \delta_{R}\left[h\left(\mathbf{v} \cdot \mathbf{n}_{k}\right)\right] \varphi^{(k)} \delta_{R}\left[h\left(\vec{v} \cdot \mathbf{n}_{k}\right)\right] \varphi^{(k)} \\
& \rightarrow \frac{R}{\pi} \sum_{k=1}^{8} \delta\left[h\left(\mathbf{v} \cdot \mathbf{n}_{k}\right)\right]\left(\varphi^{(k)}\right)^{2} \\
& =\frac{R}{\pi} \sum_{k=1}^{8} \delta\left[1-\frac{\delta \mu}{q \mathbf{v} \cdot \mathbf{n}_{k}}\right]\left(\varphi^{(k)}\right)^{2} \\
& =\frac{R^{2}}{\pi^{2}} k_{R} \sum_{k=1}^{8} \delta\left[\mathbf{v} \cdot \mathbf{n}_{k}-\frac{\delta \mu}{q}\right]\left(\varphi^{(k)}\right)^{2},
\end{aligned}
$$

with

$$
k_{R}=\frac{\pi|\delta \mu|}{q R} .
$$

Therefore one gets

$$
\begin{aligned}
\mathcal{L}_{\text {eff }}(p)= & -\frac{\mu^{2} k_{R}}{2 \pi^{2} f^{2}} \sum_{i, j=1}^{3} \sum_{k=1}^{8} \sum_{\mathbf{v}} \delta\left\{\mathbf{v} \cdot \mathbf{n}_{k}-\frac{\delta \mu}{q}\right\} \\
& \times V_{\mu} \widetilde{V}_{\nu} \epsilon_{i}^{(k)} \epsilon_{j}^{(k)} p_{\mu} \phi^{(i)} p_{\nu} \phi^{(j)} .
\end{aligned}
$$

The integration over the Fermi velocities requires special attention. We use the result

$$
\sum_{k=1}^{8} \epsilon_{i}^{(k)} \epsilon_{j}^{(k)}=8 \delta_{i j}
$$

this fixes the constant multiplying the time-derivative term in the effective Lagrangian [taking into account Eq. (4.21)] at the value

$$
\frac{8 \mu^{2} k_{R}}{2 \times 4 \pi^{2} f^{2}}
$$

Therefore we obtain canonical normalization for the kinetic term, provided

$$
f^{2}=\frac{8 \mu^{2} k_{R}}{4 \pi^{2}}
$$

The parameters $a, b$, and $c$ of the effective Lagrangian (5.95) can now be evaluated and we find (Casalbuoni, Fabiano, et al., 2002) 
$\mathrm{f}(\mathrm{R})$

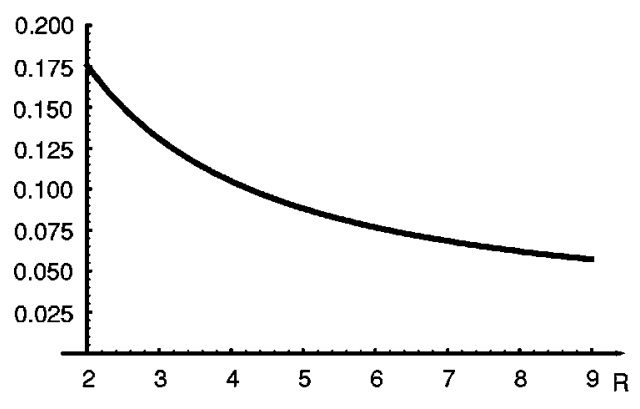

$$
\begin{aligned}
& \mathcal{L}_{\mathrm{eff}}(p)=\frac{1}{2}\left(p^{0^{2}} \phi^{(i)^{2}}-\frac{1}{8} \beta_{l m}^{i j} p^{l} p^{m} \phi^{(i)} \phi^{(j)}\right) \\
& =\frac{1}{2}\left(p^{0^{2}} \phi^{(i)^{2}}-\frac{|\mathbf{p}|^{2}}{12} \phi^{(i)^{2}}\right. \\
& \left.-\frac{3 \cos ^{2} \theta_{q}-1}{6} \sum_{i<j=1,2,3} p^{i} \phi^{(i)} p^{j} \phi^{(j)}\right),
\end{aligned}
$$

i.e., comparing with Eq. (5.95),

$$
a=\frac{1}{12}, \quad b=0, \quad c=\frac{3 \cos ^{2} \theta_{q}-1}{12} .
$$

\section{F. Gluon dynamics in the LOFF phase}

\section{One-plane-wave structure}

In this section and in the subsequent one we wish to derive the effective Lagrangian for the gluons of the unbroken $\mathrm{SU}(2)_{c}$ subgroup of the two-flavor LOFF phase. To begin with, we assume the crystal structure given by a plane wave and we neglect the vector condensate, so that we write

$$
\Delta_{\mathrm{eff}}=\frac{\Delta \pi}{R} \delta_{R}[h(\mathbf{v} \cdot \hat{\mathbf{n}})] .
$$

The effective action allows the evaluation of the oneloop diagrams with two external gluon lines and internal quark lines similar to those in Fig. 20. If we write

$$
\Pi_{a b}^{\mu \nu}(p)=\Pi_{a b}^{\mu \nu}(0)+\delta \Pi_{a b}^{\mu \nu}(p),
$$

then the Meissner mass vanishes,

$$
\Pi_{a b}^{i j}(0)=0
$$

and the Debye screening mass is nonvanishing,

$$
m_{D}=\frac{g \mu}{\pi} \sqrt{1+\frac{\cos \theta_{a}-\cos \theta_{b}}{2}}
$$

where $\cos \theta_{a}$ and $\cos \theta_{b}\left(-1 \leqslant \cos \theta_{a} \leqslant \cos \theta_{b} \leqslant 1\right)$ are the solutions of the equation

$$
|h(\cos \theta)|=\frac{\pi}{2 R}
$$

Next we consider $\delta \Pi_{a b}^{\mu \nu}(p)$. The only nonvanishing contribution to $\delta \Pi_{a b}^{\mu \nu}(p)$ comes from the pairing region, i.e., where $\Delta_{\text {eff }} \neq 0$. In the approximation of small momenta $(|p| \ll \Delta)$ one finds (Casalbuoni, Gatto, et al., 2002a)

$$
-\delta \Pi_{a b}^{\mu \nu}(p)=\delta_{a b} \frac{\mu^{2} g^{2}}{12 \pi^{2}} \sum_{\mathbf{v} ; \text { pairing }} \frac{V^{\mu} V^{\nu}(\widetilde{V} \cdot p)^{2}-\widetilde{V}^{\mu} V^{\nu}(V \cdot p \widetilde{V} \cdot p)+V \leftrightarrow \widetilde{V}}{\Delta_{\mathrm{eff}}^{2}} .
$$

That is,

$$
-\delta \Pi_{a b}^{00}(p)=\delta_{a b} \frac{g^{2} \mu^{2}}{3 \pi^{2}} \sum_{\mathbf{v} ; \text { pairing }} \frac{v_{i} v_{j}}{\Delta_{\mathrm{eff}}^{2}} p_{i} p_{j}=\delta_{a b} \frac{g^{2} \mu^{2} R^{2}}{3 \Delta^{2} \pi^{4}} \int_{\text {pairing }} \frac{d \cos \theta d \phi}{8 \pi} \frac{v_{i} v_{j}}{\left\{\delta_{R}[h(\cos \theta)]\right\}^{2}} p_{i} p_{j},
$$

where $\mathbf{v}=(\sin \theta \cos \phi, \sin \theta \sin \phi, \cos \theta)$. The integration domain is defined by $\cos \theta_{a}<\cos \theta<\cos \theta_{b}$. Therefore one gets

$$
-\delta \Pi_{a b}^{00}(p)=\delta_{a b} k\left[f(R) p_{\perp}^{2}+g(R) p_{\|}^{2}\right],
$$

where $k$ is given by ( $\Delta_{0}$ is the homogeneous condensate)

$$
k=\frac{g^{2} \mu^{2}}{18 \pi^{2} \Delta_{0}^{2}},
$$

and

$$
f(R)=\frac{3}{4} \int_{\text {pairing }} d \cos \theta\left(1-\cos ^{2} \theta\right),
$$




$$
g(R)=\frac{3}{2} \int_{\text {pairing }} d \cos \theta \cos ^{2} \theta
$$

are functions of the parameter $R$, shown in Fig. 22.

It is interesting to note the anisotropy of the polarization tensor exhibited by these results. We always have $g>f$; for large $R$, and neglecting $\delta \mu / \mu$ corrections, we find approximately

$$
\frac{g(R)}{f(R)} \rightarrow \frac{2}{\left(\frac{q}{\delta \mu}\right)^{2}-1} .
$$

Let us finally write down the remaining components of the polarization tensor. From Eq. (5.120) we get

$$
\begin{aligned}
-\Pi_{a b}^{i j}(p)= & \delta_{a b} \frac{g^{2} \mu^{2}}{3 \pi^{2}} \sum_{\mathbf{v} ; \text { pairing }} \frac{v_{i} v_{j}}{\Delta^{2}} p_{0}^{2} \\
= & k p_{0}^{2}\left[f(R)\left(\delta_{i 1} \delta_{j 1}+\delta_{i 2} \delta_{j 2}\right)\right. \\
& \left.+g(R) \delta_{i 3} \delta_{j 3}\right]
\end{aligned}
$$

and

$$
\begin{aligned}
-\Pi_{a b}^{0 i}(p)= & k p_{0} p^{j}\left[f(R)\left(\delta_{i 1} \delta_{j 1}+\delta_{i 2} \delta_{j 2}\right)\right. \\
& \left.+g(R) \delta_{i 3} \delta_{j 3}\right] .
\end{aligned}
$$

These results complete the analysis of the LOFF model in the one-plane-wave approximation. From $\Pi_{a b}^{\mu \nu}$ we get the dispersion law for gluons at small momenta. The Lagrangian at one loop is ${ }^{6}$

$$
\mathcal{L}=-\frac{1}{4} F_{a}^{\mu \nu} F_{\mu \nu}^{a}-\frac{1}{2} \Pi_{a b}^{\mu \nu} A_{\mu}^{a} A_{\nu}^{b}
$$

(sum over the repeated color indices $a, b=1,2,3$ ). Introducing the fields $E_{i}^{a} \equiv F_{0 i}^{a}$ and $B_{i}^{a} \equiv i \varepsilon_{i j k} F_{j k}^{a}$, and using Eqs. (5.122), (5.127), and (5.128), we can rewrite the Lagrangian (5.129) as

$$
\mathcal{L}=\frac{1}{2}\left(\epsilon_{i j} E_{i}^{a} E_{j}^{a}-B_{i}^{a} B_{i}^{a}\right)+\frac{1}{2} m_{D}^{2} A_{a}^{0} A_{a}^{0},
$$

where

$$
\boldsymbol{\epsilon}_{i j}=\left(\begin{array}{ccc}
1+k f(R) & 0 & 0 \\
0 & 1+k f(R) & 0 \\
0 & 0 & 1+k g(R)
\end{array}\right) .
$$

This means that the medium has a nonisotropic dielectric tensor $\epsilon$ and a magnetic permeability $\lambda=1$. These results were obtained by taking the total momentum of the Cooper pairs along the $z$ direction. Therefore we distinguish the dielectric constant along the $z$ axis, which is

$$
\epsilon_{\|}=1+k g(R),
$$

and the dielectric constant in the plane perpendicular to the $z$ axis,

\footnotetext{
${ }^{6}$ We do not include here the three- and four-gluon vertices, which can be handled as was done by Casalbuoni, Gatto, et al. (2002b), with the result that the local gauge invariance of the one-loop Lagrangian is satisfied.
}

$$
\epsilon_{\perp}=1+k f(R) .
$$

This means that the gluon speed in the medium depends on the direction of propagation of the gluon. Along the $z$ axis the gluon velocity is

$$
v_{\|} \simeq \frac{1}{\sqrt{k g(R)}}
$$

while for gluons that propagate in the $x-y$ plane we have

$$
v_{\perp} \simeq \frac{1}{\sqrt{k f(R)}},
$$

and in the limit of large $R$, and neglecting $\delta \mu / \mu$ corrections,

$$
v_{\|} \rightarrow \frac{1}{\sqrt{2}} \tan \theta_{q} v_{\perp}
$$

with $\cos \theta_{q}$ defined in Eq. (5.37).

\section{Cubic structure}

The condensate in the cubic-structure case is given by Eq. (5.75), so we shall use the results of Sec. V.A with $\Delta_{\text {eff }}$ given by Eq. (5.78). The calculations are similar to the previous case and, similarly, the $\mathrm{SU}(2)_{c}$ gluons have vanishing Meissner mass and exhibit partial Debye screening. However, the dispersion law of the gluons is different.

As a matter of fact, we write the one-loop Lagrangian for the $\mathrm{SU}(2)_{c}$ gluons as

$$
\mathcal{L}=\frac{1}{2}\left(E_{i}^{a} E_{i}^{a}-B_{i}^{a} B_{i}^{a}\right)+\delta \mathcal{L},
$$

with

$$
\delta \mathcal{L}=-\frac{1}{2} \Pi_{a b}^{\mu \nu} A_{\mu}^{a} A_{\nu}^{b} .
$$

In the approximation $|p| \ll \Delta, \delta \Pi_{a b}^{\mu \nu}$ is again given by Eq. (5.120), but now $\Delta_{\text {eff }}$ is given by Eq. (5.78). One gets

$$
\begin{aligned}
\delta \mathcal{L} \equiv & E_{i}^{a} E_{j}^{b} \delta_{a b} \frac{g^{2} \mu^{2}}{6 \pi^{2}} \int_{\text {pairing }} \frac{d \cos \theta d \phi}{8 \pi} \frac{v_{i} v_{j}}{\Delta_{\text {eff }}^{2}} \\
& +A_{0}^{a} A_{0}^{b} \delta_{a b} \frac{g^{2} \mu^{2}}{4 \pi^{2}} \int_{\text {blocking }} d \cos \theta .
\end{aligned}
$$

Evaluating the integrals one finds

$$
\mathcal{L}=\frac{1}{2}\left(\widetilde{\epsilon}_{i j} E_{i}^{a} E_{j}^{a}-B_{i}^{a} B_{i}^{a}\right)+\frac{1}{2} M_{D}^{2} A_{0}^{a} A_{0}^{a},
$$

with the tensor $\tilde{\epsilon}^{i j}$ given by

$$
\tilde{\epsilon}_{i j}=\delta_{i j}[1+k t(R)]
$$

and

$$
M_{D}=\frac{g \mu}{\pi} \sqrt{1+8 \frac{\cos \theta_{a}-\cos \theta_{b}}{2}},
$$




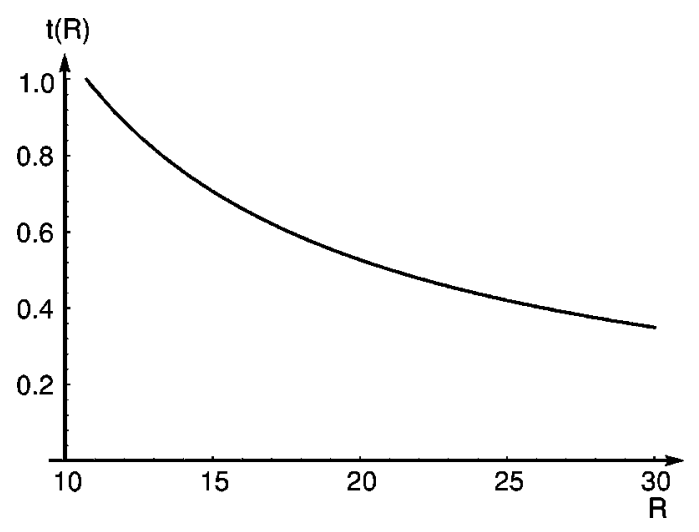

FIG. 23. Plot of the function $t(R)$. See Eq. (5.143).

where $\cos \theta_{a, b}$ are solutions to Eq. (5.119). The tensor $\widetilde{\epsilon}^{i j}$ is isotropic. This result can be easily explained by noticing that the Lagrangian should be a quadratic function of the field strengths and should also satisfy cubic symmetry. Therefore it must be constructed by the invariants $I_{2}\left(E_{i}\right)$ and $I_{2}\left(B_{i}^{a}\right)$, which are isotropic. As shown by Casalbuoni, Gatto, et al. (2002a), $t(R)$ is given by

$$
t(R)=\frac{8}{3}[2 f(R)+g(R)] .
$$

It should also be noted that the values of the parameter $R$ for the cube and the plane wave can be different. A plot of the function $t(R)$ is given in Fig. 23.

Even if the crystalline structure is not isotropic, the dielectric properties of the medium will be isotropic and the velocity of propagation of the gluons will be the same in all directions.

\section{INHOMOGENEOUS SUPERCONDUCTIVITY IN CONDENSED MATTER, NUCLEAR PHYSICS, AND ASTROPHYSICS}

As observed in the Introduction, the main focus of this review is on the theoretical methods rather than phenomenological consequences. However, for completeness, in this section we review the different approaches developed thus far to detect the inhomogeneous phase in superconductors. The LOFF phase is expected to be ubiquitous. Therefore one might expect to find it in completely different physical systems. For obvious reasons, research in solid-state physics is much more advanced and, indeed, signals of formation of the LOFF phase have been reported in the literature. We review them in the first part of this section. In order to produce the effective exchange interaction of Eq. (2.1), a sufficiently high magnetic field is needed to produce an appreciable difference between the chemical potentials. This could be done in type-I superconductors, but the required fields are likely to destroy superconductivity altogether. This issue is discussed in Sec. VI.A. To overcome this difficulty, type-II superconductors must be used; moreover, they should be free of impurities and have a large electron mean free path; the needed requirements and the associated phenomenology are dis- cussed in Secs. VI.B and VI.C. Another way to overcome the effects of high magnetic fields that are detrimental to electron superconductivity is to use layered superconductors and magnetic fields parallel to the layers. Superconductors of this type are rather different from the ones considered in the original LOFF papers; in particular, organic superconductors are compounds with these features and are therefore good candidates. They will be discussed in Sec. VI.D, while in Sec. VI.E we briefly discuss future possible developments in the area of atomic physics. The final part of this section is devoted to phenomenological implications of the LOFF phase in nuclear physics (Sec. VI.F) and QCD (Secs. VI.G and VI.H). In particular, in this last subsection we discuss a possible role of the QCD LOFF phase in a peculiar phenomenon of pulsars, i.e., the periodic glitches in their angular velocity.

\section{A. Type-I superconductors}

In the original LOFF papers (Fulde and Ferrell, 1964; Larkin and Ovchinnikov, 1964) the difference in chemical potentials between spin-up and spin-down electrons arises from the interaction of a magnetic field with the electron magnetic dipole moments. The magnetic field can hardly be the external field $H_{\text {ext. }}$, which exerts a stronger influence on the orbital motion than on the electron spin. Therefore the inhomogeneous phase was thought to arise in nonmagnetic metals in the presence of paramagnetic impurities. Under an external field, the host impurities display ferromagnetic alignment. With decreasing temperature the material becomes a superconductor while the ferromagnetic alignment persists, leading to a constant self-consistent exchange field, proportional to the average spin of the impurities. This field is at the origin of the modulated order parameter. The value $\delta \mu_{1} \approx \Delta_{0} / \sqrt{2}$, above which the LOFF phase can exist, corresponds to a critical value of the magnetic field that can be derived as follows (Chandrasekhar, 1962; Clogston, 1962): The susceptibility of an electron gas in the normal phase at $T=0$ is

$$
\chi_{n}=\mu_{B}^{2} \rho,
$$

where $\rho=g p_{F}^{2} /\left(2 \pi^{2} v_{F}\right)$ is the density of states at the Fermi surface, $\mu_{B}$ is the Bohr magneton, and $g=2$ is the electron degeneracy factor. On the other hand, the susceptibility in the superconducting phase at $T=0$ vanishes: $\chi_{s}=0$ because, in order to polarize the superconductor, one has to break the Cooper pair, which costs energy. The free energy per unit volume $f_{s}$ of the superconductor, in the absence of paramagnetic effects, is

$$
f_{s}=f_{n}-\frac{H_{c}^{2}(T)}{8 \pi}
$$

where $f_{n}$ is the free energy of the normal phase and $H_{c}(T)$ is the critical field. Including the Pauli paramagnetism implies adding to the Hamiltonian a term

$$
-\mu_{B} \psi^{\dagger} \boldsymbol{\sigma} \cdot \mathbf{H} \psi
$$

while Eq. (6.2) becomes 


$$
f_{s}=f_{n}+\frac{\left(\chi_{n}-\chi_{s}\right) H^{2}}{2}-\frac{H_{c}^{2}(T)}{8 \pi}
$$

Therefore BCS superconductivity will survive at $T=0$ for magnetic fields satisfying

$$
H \leqslant \sqrt{\frac{H_{c}^{2}(0)}{4 \pi \chi_{n}}} \equiv H_{P}(0)
$$

Now $H_{c}^{2}(0) / 8 \pi=\rho \Delta_{0}^{2} / 4$ and therefore the Pauli limiting field at $T=0$ is

$$
H_{P}(0)=\frac{\Delta_{0}}{\sqrt{2}} \frac{1}{\mu_{B}} .
$$

From a comparison of Eqs. (6.3), (6.6), and (2.1), we see that $\delta \mu_{1}=\mu_{B} H_{P}(0)$.

For a type-I superconductor it is difficult to reach the Pauli limit (6.6) because, while $H_{P}(0)$ is typically of the order of $300 \mathrm{kOe}, H_{c}(0)$ is of the order of $1 \mathrm{kOe}$. Therefore superconductivity will be broken by the magnetic field well before the Clogston-Chandrasekhar limit is reached. This implies that the LOFF phase is unlikely to be produced by these materials, and one has to turn to type-II superconductors (Saint-James et al., 1969) because for some of these superconductors the upper critical field $H_{c 2}$ can be very high.

\section{B. "Clean" and strongly type-II superconductors}

To evaluate the possibility of the LOFF state one has to take into account not only Pauli paramagnetism of the electrons but also orbital effects (Ginzburg, 1957). Before doing that, let us first distinguish between "clean" and "dirty" superconductors (Anderson, 1959). One calls "clean" the superconductors, in which the electron mean free path $l$ is much larger than the superconducting coherence length $\xi_{0}$,

$$
l \gg \xi_{0},
$$

while "dirty" superconductors are characterized by the opposite condition, $l \ll \xi_{0}$. In clean superconductors, electrons at the Fermi surface move with velocity $\mathbf{v}_{F}$, while in dirty superconductors the electron motion is described by a diffusion equation. Dirty superconductors are characterized by the presence of impurities, which can narrow and even destroy the LOFF state (Aslamazov, 1969; Takada, 1970). Therefore materials with small $l$, e.g., $\mathrm{PbMo}_{6} \mathrm{~S}_{8}$, should not display the LOFF phase (see, for example, Decroux and Fischer, 1982). On the other hand, the so-called heavy-fermion superconductors are favored: these materials are indeed characterized by the small Fermi velocity of their quasiparticles (Rauchschwalbe, 1982); since

$$
\xi_{0}=\frac{\hbar v_{F}}{\pi \Delta}
$$

for small enough $v_{F}$ the condition (6.7) is satisfied. For example, in the heavy-fermion compound $\mathrm{UPd}_{2} \mathrm{Al}_{3}$, the superconducting coherence length is $\xi_{0} \approx 85 \AA$, which is much smaller than the electron mean free path $l$ $=700 \AA$; therefore it can be considered a clean superconductor.

To evaluate the possibility of the LOFF state in type-II superconductors, one has to take into account not only Pauli paramagnetism of the electrons, but also orbital effects. This analysis was first performed by Gruenberg and Gunther (1966). These authors followed the variational method of Werthamer et al. (1966) by making an ansatz for the condensate. In general, there is competition between orbital and paramagnetic effects, the former trying to organize a structure of Abrikosov vortices and the latter a periodic LOFF structure. Orbital effects therefore reduce the possibility of a LOFF state which can exist only for sufficiently high $H_{c 2}$. At $T=0$ for clean type-II superconductors with an isotropic dispersion law, the LOFF state can persist provided

$$
\alpha=\sqrt{2} \frac{H_{c 2}(0)}{H_{P}(0)}>1.8 \text {. }
$$

Here $\alpha$ is the parameter first introduced by Maki (1964), $H_{c 2}(0)$ is the Gor'kov upper critical field at $T=0$ in the absence of paramagnetic effects (Gor'kov, 1960), and $H_{P}(0)$ is the Pauli limiting field defined in Eq. (6.6).

In conclusion, good experimental conditions for observing the LOFF state should be provided by a clean superconductor with a large $\alpha$ value. These features are not easily found in the most common superconductors and therefore experimental investigations consider unconventional superconductors, e.g., heavy-fermion, organic, or high- $T_{c}$ superconductors. As a matter of fact, many of these materials are strongly type-II superconductors, which means that the condition (6.9) can be satisfied. Moreover, they often have a layered structure, which implies that, if the magnetic field is applied parallel to the layers, the orbital effect is minimum and the Zeeman effect, on which the LOFF phase is based, is dominant.

Very clean and simultaneously strong type-II superconductors should be more easily realizable in $d$-wave materials like the high- $T_{c}$ cuprates and organic superconductors like $\kappa-(\mathrm{ET})_{2}$ or $\lambda-(\mathrm{ET})_{2}$ salts. They will be discussed in more detail below; suffice it to say here that in $d$-wave superconductors the region of the LOFF phase is much more extended than in $s$-wave superconductors. The analysis of Maki and Won (1996), where this conclusion was drawn, has been extended by Won and Maki (2002) to the calculation of the LOFF free energy, specific heat, and magnetic susceptibility. In particular, for these layered $d$-wave superconductors the energetically favored structure at $T=0$ is found to be

$$
\Delta(x, y) \propto \cos q x+\cos q y .
$$

Other materials in which the possible existence of LOFF phases has been investigated are ferromagnetic metals or alloys (Pickett et al., 1999; Dyugaev et al., 2001). The study of the possible coexistence of ferromagnetism and superconductivity was initiated by Ginzburg (1957), who noted that, though the two orderings can in principle coexist, their simultaneous presence is 
practically impossible under ordinary conditions. As a matter of fact, the presence in ferromagnets of a spontaneous magnetization $M_{0}$ produces at $T=0$ an internal magnetic induction $B_{0}=4 \pi M_{0}$ even in the absence of an external magnetic field. For superconductivity to exist, $B_{0}$ should be smaller than the lower critical field at $T$ $=0$, in the absence of ferromagnetism:

$$
B_{0} \leqslant H_{c 1}^{0}(0) \text {. }
$$

However, the induction $B_{0}$ at $T=0$ is of the order of 10 kOe (e.g., 22, 18.5, 6.4, $24.8 \mathrm{kOe}$, respectively, for Fe, $\mathrm{Co}, \mathrm{Ni}, \mathrm{Gd}$ ), while the critical field is in general much smaller, of the order of a few kOe or less. Superconductivity in ferromagnets is therefore difficult unless condition (6.11) is rendered possible by special situations such as reduced size of the sample, with dimensions of the order of the penetration depth, or formation of a vortex phase in type-II superconductors, which locally screens the internal magnetic induction, allowing an exception to Ginzburg's negative conclusion (Krey, 1973). As a matter of fact, superconductivity was recently reported in the ferromagnetic alloy $\mathrm{RuSr}_{2} \mathrm{GdCu}_{2} \mathrm{O}_{8}$ (Bernhard, 1999; Hadjiev et al., 1999; Pringle et al., 1999; Tallon et al., 1999). This layered material first becomes ferromagnetic at $T=132 \mathrm{~K}$; superconductivity appears at $T$ $=35-40 \mathrm{~K}$, and finally, at $T=2.6 \mathrm{~K}$, Gd ions acquire an antiferromagnetic order. The theoretical study of Pickett et al. (1999) confirms these reports, but suggests that the superconducting phase is of the LOFF type because the coupling between ferromagnetism and superconducting layers appears to be sufficiently weak to permit superconductivity, but strong enough to require the inhomogeneous phase. In a similar context, Dyugaev et al. (2001) consider the possibility of creating the LOFF phase using ferromagnetic materials instead of nonmagnetic bulks with paramagnetic impurities as in the original LOFF papers. The impurities create not only an exchange interaction, but also an electromagnetic interaction. Using nuclear ferromagnetism, as they propose, would reduce the latter, since the effective field would be proportional to the nuclear magneton and not to the Bohr magneton. They show that in some metals, e.g., $\mathrm{Rh}$ and $\mathrm{W}$, the BCS condensate embedded in a matrix of ferromagnetically ordered nuclear spins should manifest the LOFF phase.

All the proposals we have discussed so far are rather different from the one discussed in the original LOFF papers. An extension of the LOFF analysis to these materials and to unconventional superconductors is beyond the scope of the present review. We shall therefore limit our presentation to a brief survey of the experimental results, referring the interested reader to the specialized literature (Murthy and Shankar, 1995; Gegenwart, 1996; Shimahara et al., 1996; Samohkin, 1997; Shimahara, 1998b; Pickett et al., 1999; Agterberg and Yang, 2001; Yang, 2001).

\section{Heavy-fermion superconductors}

The first experimental studies of the LOFF phase used heavy-fermion compounds such as $\mathrm{CeRu}_{2}$ (Huxley et al., 1993), $\mathrm{UPd}_{2} \mathrm{Al}_{3}$ (Gloos et al., 1993), and $\mathrm{UBe}_{13}$ (Thomas et al., 1996). For all these materials the conditions for the formation of the LOFF state are met. For example, $\mathrm{CeRu}_{2}$ is in a metallurgically clean state; moreover, it exhibits extreme type-II behavior because the Ginzburg-Landau parameter, which discriminates between the two types of superconductors (Saint-James et al., 1969), has the value $\kappa=16$. As another example, the compound $\mathrm{UPd}_{2} \mathrm{Al}_{3}$ used by Gloos et al. (1993) is characterized by a rather large value of the parameter $\alpha$ in Eq. (6.9), i.e., $\alpha=2.4$, while also being a very clean superconductor. To cite another result, in the analysis of a high-quality single crystal of $\mathrm{UBe}_{13}$ (Thomas et al., 1996), the very high value $H_{c 2}(0) \simeq 140 \mathrm{kOe}$ was reached. All these experimental results are, however, inconclusive. In a critical analysis of the experiment of Gloos et al. (1993), Norman (1993) shows that the computed Gor'kov upper critical field does not correspond to the experimental results reported there. For further analysis of the compound $\mathrm{UPd}_{2} \mathrm{Al}_{3}$ see Yin and Maki (1993) and Schimanski (1994). In the case of $\mathrm{CeRu}_{2}$, Tenya et al. (1999) shows that the observed effects can be explained by flux-pinning mechanisms involving disorder. Modler et al. (1996) makes a comparative study of high-quality single crystals of $\mathrm{UPd}_{2} \mathrm{Al}_{3}$ and $\mathrm{CeRu}_{2}$ in the mixed state. The order parameter exhibits a periodic array of nodal planes perpendicular to the Abrikosov vortex lines. In the mixed state, the pinning force is very weak; however, the authors find, for $H>10 \mathrm{kOe}$ and $T$ $<0.9 T_{c}$, a first-order transition to a state characterized by strong pinning, which might be interpreted as the formation of a LOFF state. The mechanism by which Abrikosov vortex lines in type-II superconductors are pinned to the vortex cores is similar to the one that pins vortex lines to nonsuperfluid neutrons in a rotating superfluid within neutron stars. This will be explained in more detail in Sec. VI.H in connection with the possible role of the QCD LOFF state in the physics of pulsars.

\section{Two-dimensional, quasi-two-dimensional, and organic superconductors}

As we already mentioned, the paramagnetic effect can dominate if the superconducting bulk has a layered structure and the magnetic field acts parallel to it, because in this case the orbital upper critical field can be extremely high and the breaking due to the spin interaction is most significant. The importance of twodimensionality in favoring the LOFF state was first observed by Bulaevskii $(1973,1974)$, who took into account both the orbital and the spin effect and who calculated the upper critical field $H_{c 2}(T)$. Buzdin and Kulic (1984) carried out the analysis near the tricritical point. For the same reason, quasi-one-dimensional compounds were also discussed (Buzdin and Tugushev, 1983; Buzdin and Polonski, 1987; Dupuis et al., 1993; Dupuis, 1995), even though the results of Shimahara (1998a) indicate that the $2 \mathrm{D}$ structures are favored over the $1 \mathrm{D}$ ones. 
These results were generalized to arbitrary temperature and $d$-wave superconductivity by Shimahara and Rainer (1997). The main result of this paper is that the critical field curve $H_{c 2}(T)$ is nonmonotonic and consists of different pieces corresponding to different Landau levels, characterized by $n>0$. In contrast, the GinzburgLandau theory would predict the pair wave function to be in the lowest energy Landau level, with $n=0$ at $H_{c 2}$. Shimahara (1998a) studied the most favored structure for a 2D LOFF crystal with a cylindrical Fermi surface. First, the author found that, in general, 2D structures are favored over 1D ones; second, he found that the favored crystalline structure changes with $T$. For $s$-wave superconductivity, the results are as follows: at high temperatures, the antipodal pair condensate

$$
\Delta(\mathbf{r}) \propto 2 \cos \mathbf{q} \cdot \mathbf{r}
$$

is favored. This was the result found by Larkin and Ovchinnikov (1964) in three dimensions at $T=0$. With decreasing temperature, other structures become favored: first the triangle, then the square, and finally, at low temperatures, the hexagon. For $d$-wave pairing at high temperature, again Eq. (6.12) is favored, while at low temperature the square dominates; on the other hand, at intermediate temperatures the phase transition should be first order. The result at $T=0$ has been confirmed by Won and Maki [2002; see Eq. (6.10)]. As shown by Lebed' (1986), the quasi-2D superconductors can be treated as essentially $2 \mathrm{D}$, and therefore the results of Shimahara (1998a) should also hold for quasi-2D compounds, provided the external field is sufficiently strong and is kept parallel to the superconducting layer.

Klein et al. (2000) consider a layered superconductor in a magnetic field of arbitrary orientation with respect to the conducting plane. The calculation is based on the quasiclassical Eilenberger equations (Eilenberger, 1968; Alexander et al., 1985) and allows elucidation of the structure of the stable states below $H_{2 c}$, minimizing the free energy. The stable states are neither pure LOFF states nor pure Abrikosov vortex states, but are twodimensional periodic structures or quasi-onedimensional structures in which LOFF domains are separated by vortex chains. Barzykin and Gor'kov (2002) address 2D surface superconductivity in the presence of intense magnetic fields parallel to the surface. The spin-orbit interaction at the surface changes the properties of the LOFF state; they find that strong spinorbit interactions significantly broaden the range of parameters where the LOFF phase can exist and produce periodic superconducting stripes running along the field direction on the surface.

Organic superconductors are good candidates for the formation of the LOFF state for the reasons mentioned above: (i) they have narrow electron bands and therefore they are, in principle, clean type-II superconductors; and (ii) due to their low dimensionality, the orbital pair-breaking effect is suppressed for magnetic fields parallel to the layers they form. For these reasons, they have been discussed by several authors, including Lebed' (1986), Gor'kov and Lebed' (1987), Dupuis et al.
(1993), Burkhardt and Rainer (1994), Shimahara (1994, 1997), and Dupuis (1995). It is interesting to note that, in general, thermodynamic signatures are chosen to detect the transition from the homogeneous BCS phase to the LOFF phase. This, however, can give ambiguous results, since the signatures can be produced by different kinds of phase transitions. Therefore Yang and Agterberg (2000) propose the use of the Josephson effect. According to this analysis, at the Josephson junction between two superconductors, one in the BCS and the other in the LOFF phase, the Josephson current is suppressed.

As discussed by Shimahara and Rainer (1997), the upturn of the upper critical field $d^{2} H_{c} / d T^{2}>0$ is a common feature in the LOFF state in quasi-2D systems and is due to a Fermi-surface effect. Investigations of the sensitivity of the LOFF state to the shape of the Fermi surface include those of Aoi et al. (1974) and Shimahara $(1994,1997)$. This upturn and a first-order transition below the critical field have been observed in the organic compound $\kappa$-(BEDT-TTF $)_{2} \mathrm{Cu}(\mathrm{NCS})_{2}$, a quasi-twodimensional organic superconductor examined by a number of authors (Nam et al., 1999; Houzet and Buzdin, 2000; Ishiguro, 2000; Singleton et al., 2000, 2001; Symington et al., 2001). A similar compound is studied by Goddard et al. (2002). These studies give some evidence of the formation of the LOFF state. For example, Singleton et al. (2000) studied resistance and magnetic behavior of single crystals of this superconductor in magnetic fields up to $33 \mathrm{~T}$ and at temperatures between 0.5 and $11 \mathrm{~K}$. When the magnetic field lies precisely in the quasi-2D planes of the material, they find evidence for a phase transition from the superconducting mixed state into a LOFF state, manifested as a change in the rigidity of the vortex system. Manalo and Klein (2000) compare the theoretical anisotropic upper critical field $H_{c}$ of a quasi-two-dimensional $d$-wave superconductor with recent $H_{c 2}$ data for $\kappa$-(BEDT-TTF $)_{2} \mathrm{Cu}(\mathrm{NCS})_{2}$ and find agreement in both the angular and the temperature dependence of $H_{c}$. According to these authors, this supports the suggestion that the LOFF phase exists in this material for exactly plane-parallel orientation of the magnetic field.

Uji (2001) reported field-induced superconductivity in an organic superconductor, $\lambda$-(BETS $)_{2} \mathrm{FeCl}_{4}$ [BETS $=$ bis( ethylenedithio) tetraselenafulvalene]. A possible mechanism to create field-induced superconductivity is the Peter-Jaccarino effect (Jaccarino and Peter, 1962). However, the upwards convex nature of the lower critical field as a function of the temperature casts doubts on this interpretation. Therefore some authors, e.g., Balicas et al. (2001), have proposed that these results can be interpreted as evidence of the formation of the LOFF state. These results were reviewed by Houzet et al. (2002) and Shimahara (2002). In particular, in the latter paper, an experimental phase diagram of the fieldinduced superconductivity in this organic compound was theoretically reproduced by a combination of the LOFF state and the Jaccarino-Peter mechanism. Tanatar et al. (2002) discuss whether the LOFF state has been observed via thermal conductivity $\kappa(H)$ in the quasi-two- 
dimensional organic superconductor $\lambda$-(BETS $)_{2} \mathrm{GaCl}_{4}$. For clean samples the behavior of $\kappa(H)$ is similar to that expected for a second-order phase transition and is consistent with the formation of a LOFF phase.

\section{E. Future developments}

The superconducting LOFF phase might be realized even if the difference in chemical potentials of two species were not generated by a magnetic field acting on electron spins. Apart from nuclear physics and pulsars, to be discussed below, another context might be offered by an ultracold quantum degenerate Fermi gas of atoms comprising two hyperfine states. The experimental investigations of ultracold gases were first dedicated to the study of Bose-Einstein condensation (Anderson et al., 1995; Davis et al., 1995; Bradley et al., 1997; Fried et al., 1998); subsequently these techniques were extended to magnetically trapped ultracold alkali Fermi gases or to gases with coexisting Bose-Einstein condensate and Fermi gas (Schreck et al., 2001; Modugno et al., 2002; Roati et al., 2002). In particular, two-state mixtures of ultracold gases have been employed, with ${ }^{40} \mathrm{~K}$ vapors (DeMarco and Jin, 1999; DeMarco et al., 2001), or ${ }^{6} \mathrm{Li}$ (O'Hara et al., 2001; Granade et al., 2002), or a mixture of ${ }^{6} \mathrm{Li}$ and ${ }^{7} \mathrm{Li}$ (Mewes et al., 1999). Future developments could lead to the observation of superconductivity and Cooper fermion pair condensation in these systems. As discussed by Combescot (2001), it is quite likely that the two hyperfine states would have different atomic populations, since at the moment there are no known fast relaxation mechanisms to equalize the two atomic populations. Therefore superconductivity for two-state ultracold Fermi gases is likely to be of the LOFF type. Combescot (2001) has performed a theoretical study of ${ }^{6} \mathrm{Li}$ under the above-mentioned conditions; he considers not only $s$-wave interactions, but also an anisotropic term induced by density fluctuation exchange and shows that the range where the LOFF phase is realized increases with the increasing role of the anisotropic term. This is an interesting theoretical development, which adds new interest to the experimental investigations of ultracold atomic Fermi gases. It remains to be seen, however, if such a possibility is actually realized in $\mathrm{Na}$ ture.

\section{F. LOFF phase in nuclear physics}

Neutron-proton pair correlations and the possibility of $n$ - $p$ Cooper-pair condensation are at present studied in several different contexts, from heavy-ion collisions to astrophysics. They have been investigated, using the BCS theory, in infinite nuclear matter (Alm et al., 1990, 1993, 1996; Vonderfecht et al., 1991; Baldo et al., 1992; Sedrakian et al., 1997) and by mean-field effective interactions in finite nuclei. In several cases nuclear matter is highly asymmetric, with proton concentration at most $30-40 \%$ in supernova matter and $10 \%$ in neutron stars. These asymmetries are detrimental to nucleon superconductivity; on the other hand, for weakly asymmetric states, fermion condensation is indeed possible. For example, weakly isospin-asymmetric nuclear matter favors the formation of Cooper pairs in the ${ }^{3} S_{1}{ }^{-}{ }^{3} D_{1}$ channel, due to the presence of a tensor force; gaps are of the order of $10 \mathrm{MeV}$. Condensation in this channel might be relevant for low-density bulk matter such as the dilute nuclear matter in supernovas. On the other hand, there is no evidence of large-gap isospin singlet pairing in ordinary nuclei, which might be explained by the presence of a spin-orbit interaction (Goodman, 1999; MartinezPinedo et al., 1999). Sedrakian and Lombardo (2000) study the dependence of the gap on both the isospin asymmetry $\alpha_{n p}=\left(\rho_{n}-\rho_{p}\right) / \rho$ and the temperature, using realistic nuclear interactions. For small asymmetries the gap develops a maximum at a certain intermediate temperature; for large asymmetries the superconducting phase exists only at finite temperature, because the smearing effect of the temperature on the Fermi surfaces favors condensation. At higher values of $\alpha_{n p}$ ( $\simeq 0.11$ in their model), pairing is no longer possible.

Also in the context of isospin-asymmetric nuclear matter, it is possible to have a transition from the BCS state to a LOFF phase instead of to the normal state (Sedrakian et al., 1997; Sedrakian, 2001; Isayev, 2002). Sedrakian (2001) studies the possibility of a spatially inhomogeneous condensate in asymmetric nuclear matter. Condensation is possible in different channels. The isospin-triplet channels are favored for large enough asymmetries; more exactly, the channel ${ }^{1} S_{0}$ dominates at low densities, and the channel ${ }^{3} P_{2}-{ }^{3} F_{2}\left(\right.$ or $\left.{ }^{1} P_{2}\right)$ at high densities. For weak asymmetries the dominant channels are the isospin singlets ${ }^{3} S_{1-}{ }^{3} D_{1}$ (low density) and ${ }^{3} D_{2}$ (high density). Sedrakian considers the case of low density. As the isospin singlet ${ }^{3} S_{1-}{ }^{3} D_{1}$ has a strength much larger than the isospin triplet ${ }^{1} S_{0}$, he neglects the latter. The interaction is modeled by the Paris nucleon-nucleon potential. The gap equations are solved numerically and have nontrivial solutions for nonvanishing total momentum of the pair $P$. The LOFF phase is favored for $\alpha_{n p}>0.25$ and $P=0.3 p_{F}$, independent of $\alpha_{n p}$. For $\alpha_{n p}>0.37$, pairing exists only in the presence of nonvanishing $P$. The maximal values of $\alpha_{n p}$ and $P$ compatible with the LOFF state are 0.41 and $0.3 p_{F}$, respectively (the actual values are indicative, as a refinement of the treatment of the nuclear interaction may change them by a factor as large as 3 ). The results are obtained at $T=3 \mathrm{MeV}$. From the BCS to the LOFF phase, the phase transition is first order, while from the LOFF to the normal state, it is second order. No attempt is made to determine the most favored crystalline structure.

Under hypotheses similar to those of Sedrakian, Isayev (2002) studied the effect of coupling between the isospin singlet and isospin triplet, since at low densities pairing between these two channels may be important (Akhiezer et al., 1999). Isayev goes beyond the approximation of a "bare" nucleon interaction by using the Fermi-liquid phenomenological approach (Akhiezer et al., 1994) and thereby finds interesting peculiarities at $T=0$. First, the triplet-singlet channel turns out to be 
energetically favored; second, the phase transition from the LOFF to the normal state can be of first order, depending on the nature of the nucleon interaction. While still model dependent, these investigations of the LOFF phase in nuclear interactions are interesting, as they offer, in principle, a different path to the LOFF phase. To be closer to phenomenology, one should consider more complicated structures, such as, for example, hyperonrich matter. Alternatively, the modulation of the order parameter might be caused by Pauli paramagnetism due to strong magnetic fields in highly magnetized neutron stars (magnetars). In this case one could have a splitting in the Fermi surfaces of a nucleon pair in the $I=1, L$ $=0$ channel (Sedrakian, 2001).

\section{G. Why color LOFF superconductivity could exist in pulsars}

In this subsection and in the next we shall be interested in some numerical estimates of the values of the parameters needed for the LOFF phase to occur in pulsars. In general, color superconductivity in quark matter might be realized in compact stars. This expectation follows from the following two facts.

First, the BCS critical temperature is given by

$$
T_{c}=0.57 \Delta_{B C S}
$$

and, in QCD, $\Delta_{B C S}$ is expected to range between 20 and $100 \mathrm{MeV}$. This estimate arises from weak-coupling calculations $^{7}$ which are valid only at asymptotically high chemical potentials $\mu \gg 10^{8} \mathrm{MeV}$ (Rajagopal and Shuster, 2000), and from models with parameters adjusted to reproduce the physics at zero densities. ${ }^{8}$ None of these calculations is valid at chemical potentials around $400 \mathrm{MeV}$, which corresponds roughly to the density of the inner core of a neutron star, as we shall see below. However, in all these cases one gets values of the gaps of the order of those quoted above.

The second fact has to do with the thermal history of a pulsar. The general belief is that compact stars such as pulsars are formed in the core of a supernova explosion. The temperature at the interior of a supernova is about $10^{11} \mathrm{~K}$, corresponding to $10 \mathrm{MeV}(1 \mathrm{MeV}=1.1065$ $\left.\times 10^{10} \mathrm{~K}\right)$. The star cools very rapidly by neutrino emission, with the temperature going down to $10^{9}-10^{10} \mathrm{~K}$ in about one day. Neutrino emission then dominates the cooling for one thousand years. In this period the tem-

\footnotetext{
${ }^{7}$ See Pisarski and Rischke (1999a, 1999b, 2000a, 2000b); Schafer and Wilczek (1999c); Shovkovy and Wijewardhana (1999); Son (1999); Beane and Bedaque (2000); Brown et al. (2000a, 2000b, 2000c); Evans et al. (2000); Hong et al. (2000); Hsu and Schwetz (2000); Rajagopal and Shuster (2000); Schafer (2000); Beane et al. (2001).

${ }^{8}$ Examples include the works of Alford et al. (1998); Rapp et al. (1998, 2000); Alford, Rajagopal, and Wilczek (1999); Berges and Rajagopal (1999); Carter and Diakonov (1999); Evans et al. (1999a, 1999b); Rajagopal (1999); Schafer and Wilczek (1999b); Alford (2000); Wilczek (2000); Schafer (2001).
}

perature reaches about $10^{6} \mathrm{~K}$. After this period the star cools down due to $\mathrm{x}$-ray and photon emission and in a few million years reaches a surface temperature of around $10^{5} \mathrm{~K}$. Therefore, for the greatest part of its existence, a neutron star has a temperature lower than the critical temperature, with the possibility of forming color superconducting condensates. It follows that, in this context, a compact star can be considered to be at zero temperature because its temperature is much lower than the typical BCS energy gap, $T_{\text {n.s. }} / \Delta_{B C S} \approx 10^{-6}-10^{-7}$.

We have seen previously that QCD favors the formation of BCS condensates in idealized cases, e.g., two or three massless flavors of quarks. However, in realistic cases the three quarks have different Fermi momenta due to mass differences. It is interesting to have an idea of the order of magnitude of the scales involved in the description of a neutron star with a quark core. We begin with a very crude example of a free gas of three flavor quarks, taking up and down massless quarks, and a strange quark with mass $M_{s}$ (Alford et al., 2000). Requiring that the weak interactions be in equilibrium allows us easily to determine the chemical potentials and the Fermi momenta for the quarks. We find

$$
\begin{aligned}
& \mu_{u}=\mu-\frac{2}{3} \mu_{e}, \quad p_{F}^{u}=\mu_{u}, \\
& \mu_{d}=\mu+\frac{1}{3} \mu_{e}, \quad p_{F}^{d}=\mu_{d}, \\
& \mu_{s}=\mu+\frac{1}{3} \mu_{e}, \quad p_{F}^{s}=\sqrt{\mu_{s}^{2}-M_{s}^{2},}
\end{aligned}
$$

where $\mu$ is the average chemical potential

$$
\mu=\frac{1}{3}\left(\mu_{u}+\mu_{d}+\mu_{s}\right)
$$

and $\mu_{e}$ the chemical potential of the electrons. Notice that

$$
\sum_{i=u, d, s} \mu_{i} N_{i}+\mu_{e} N_{e}=\mu N_{q}-\mu_{e} Q
$$

where

$$
N_{q}=\sum_{i=u, d, s} N_{i}, \quad Q=\frac{2}{3} N_{u}-\frac{1}{3}\left(N_{d}+N_{s}\right)-N_{e} .
$$

The chemical potential for the electrons is fixed by requiring electrical neutrality, corresponding to the condition for the grand potential $\Omega$ at zero temperature,

$$
Q=\frac{\partial \Omega}{\partial \mu_{e}}=0 .
$$

$\Omega$ is obtained from Eq. (2.60) (omitting the volume factor):

$$
\Omega=\frac{1}{\pi^{2}} \int_{0}^{p_{F}} p^{2}[E(p)-\mu] d p .
$$

In our case we get 


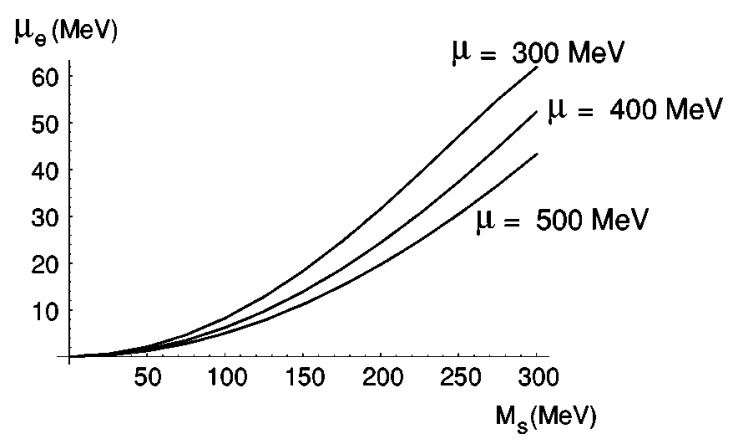

FIG. 24. The chemical potential $\mu$ of the electrons vs $M_{s}$ for three values of the average chemical potential.

$$
\begin{aligned}
\Omega= & \frac{3}{\pi^{2}} \sum_{i=u, d, s} \int_{0}^{p_{F}^{i}} p^{2}\left[E_{i}(p)-\mu_{i}\right] d p \\
& +\frac{1}{\pi^{2}} \int_{0}^{\mu_{e}} p^{2}\left(p-\mu_{e}\right) d p
\end{aligned}
$$

where

$$
E_{u, d}(p)=p, \quad E_{s}(p)=\sqrt{p^{2}+M_{s}^{2}} .
$$

Although the integral is feasible, its expression is algebraically involved and it is easier to do all of the calculations numerically. In particular, the result for the chemical potential of the electrons for different values of $\mu$ as a function of $M_{s}$ is given in Fig. 24. We can get an analytical expression by performing an expansion up to the order $M_{s}^{4} / \mu^{4}$. We get

$$
\mu_{e} \approx \frac{M_{s}^{2}}{4 \mu}
$$

and

$$
\Omega \approx-\frac{3}{4 \pi^{2}} \mu^{4}+\frac{3}{4 \pi^{2}} M_{s}^{2} \mu^{2}-\frac{7-12 \ln \left(M_{s} / 2 \mu\right)}{32 \pi^{2}} M_{s}^{4}
$$

The baryon density is obtained as

$$
\rho_{B}=-\frac{1}{3} \frac{\partial \Omega}{\partial \mu}=\frac{1}{3 \pi^{2}} \sum_{i=u, d, s}\left(p_{F}^{i}\right)^{3} .
$$

The plot of the ratio of the baryon density to the nuclear baryon density is given in Fig. 25. The nuclear baryon density has been assumed to be the inverse of the volume of a sphere of radius about $1.2 \mathrm{fm}$. Within the same approximation as before, one finds

$$
\rho_{B} \approx \frac{\mu^{3}}{\pi^{2}}\left[1-\frac{1}{2}\left(\frac{M_{s}}{\mu}\right)^{2}\right] .
$$

We note that densities in the core are of the order of $10^{15} \mathrm{~g} / \mathrm{cm}^{3}$, corresponding to a chemical potential of the order of $400 \mathrm{MeV}$, as shown in Fig. 25. In particular, let

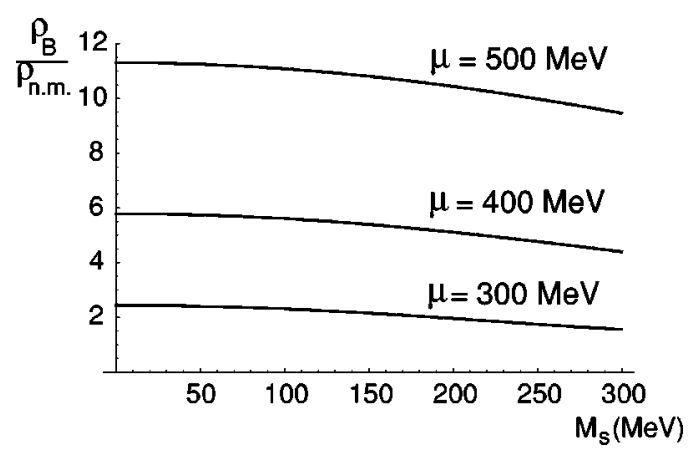

FIG. 25. The ratio of the baryon density of the free-quark gas $\rho_{B}$ to the nuclear baryon density $\rho_{n . m}$. vs $M_{s}$, for three values of the average chemical potential.

us discuss the range of values around $400 \mathrm{MeV}$ of the average chemical potential, with a strange mass of the order 200-300 MeV (the strange mass here is not the current mass, but an effective density-dependent mass). With $M_{s}=300 \mathrm{MeV}$ one finds $\mu_{e}=53 \mathrm{MeV}$ (56 MeV from the approximate equation) with Fermi momenta

$$
p_{F}^{u}=365 \mathrm{MeV}, \quad p_{F}^{d}=418 \mathrm{MeV}, \quad p_{F}^{s}=290 \mathrm{MeV}
$$

and a baryon density about 4.4 times the nuclear matter density. With $M_{s}=200 \mathrm{MeV}$ the result is $\mu_{e}=24 \mathrm{MeV}$ (25 MeV from the approximate equation) and

$$
p_{F}^{u}=384 \mathrm{MeV}, \quad p_{F}^{d}=408 \mathrm{MeV}, \quad p_{F}^{s}=357 \mathrm{MeV},
$$

and a baryon density about 5.1 times the nuclear matter density. To go to baryon densities relevant to the central core of the star, i.e., densities from six to eight times the nuclear matter density, one needs to go to higher values of $\mu$ and lower values of $M_{s}$, where the difference among the Fermi momenta is lower. This can be seen from Fig. 26, or using our approximate expression for $\mu_{e}$ :

$$
p_{F}^{u} \approx \mu-\frac{M_{s}^{2}}{6 \mu}, \quad p_{F}^{d} \approx \mu+\frac{M_{s}^{2}}{12 \mu}, \quad p_{F}^{s} \approx \mu-\frac{5 M_{s}^{2}}{12 \mu}
$$

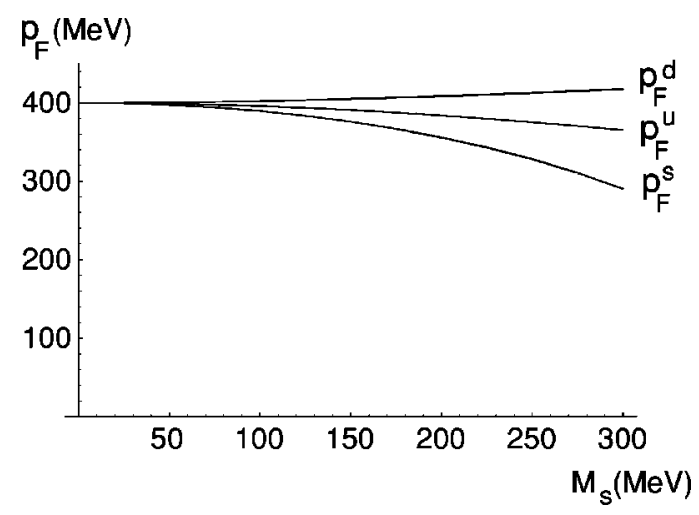

FIG. 26. The Fermi momenta $P_{f}$ of the three quarks vs $M_{s}$. 
with

$$
p_{F}^{d}-p_{F}^{u} \approx p_{F}^{u}-p_{F}^{s} \approx \frac{M_{s}^{2}}{4 \mu} .
$$

The previous results are rather general, but in order to discuss the possible astrophysical applications we need to fix a value for the BCS gap $\Delta_{0}$. Notice that we can trade the coupling constant $G$ [see Eq. (4.55)] for $\Delta_{0}$ since $G$ is fixed once we give the cutoff $\delta$. On the other hand, the equation for the chiral gap (Rajagopal and Wilczek, 2001) gives a relation between the NambuJona-Lasinio cutoff $\Lambda=\delta+\mu$, the coupling $G$, and the constituent quark mass. By taking the constituent mass around $300-400 \mathrm{MeV}$ and fixing $\Lambda$, one still has a parameter to play around with, and it is possible to get values of $\Delta_{0}$ from about $20 \mathrm{MeV}$ up to about $100 \mathrm{MeV}$. In the present case, since the typical value of $\delta \mu$ inside the LOFF window is $0.7 \Delta_{0}$ and

$$
\delta \mu=\frac{1}{2}\left(\mu_{d}-\mu_{u}\right)=\frac{1}{2} \mu_{e}
$$

we can reproduce approximately the situation illustrated at the beginning of this subsection with $M_{s}=300 \mathrm{MeV}$ by choosing $\Delta_{0}=40 \mathrm{MeV}$. With this choice the LOFF grand potential at $\delta \mu=\delta \mu_{1}$ is of the order $10^{-7} \mathrm{GeV}^{4}$, which, as we shall see in Sec. VI.H, is of the right order of magnitude to give rise to the glitch phenomenon (Alford et al., 2000). Notice also that the LOFF condensate evaluated at $\delta \mu_{1}$,

$$
\Delta_{\text {LOFF }}\left(\delta \mu_{1}\right) \approx 0.25 \Delta_{0}=10 \mathrm{MeV},
$$

is much larger than the typical temperature of a neutron star, of the order of $\mathrm{keV}$.

\section{H. Astrophysical implications of the QCD LOFF phase}

While much experimental effort is devoted to the search for the LOFF phase in condensed matter, so far there has been no comparable search in the crystalline phase of QCD. The reason is that it is difficult to create the experimental conditions of high density and low temperature for hadronic matter. The crystalline superconducting phase of quarks may, however, have results relevant for astrophysical dense systems, in particular for the explanation of pulsar glitches. Pulsars are rapidly rotating stellar objects, characterized by the presence of strong magnetic fields and by an almost continuous conversion of rotational energy into electromagnetic radiation. The rotation periods can vary from $10^{-3} \mathrm{sec}$ up to a few seconds; these periods never decrease but rather increase slowly except for occasional glitches, i.e., sudden increases of the rotational frequencies of the order of $\delta \Omega / \Omega \approx 10^{-6}$ or smaller, where $\Omega$ is the angular velocity. Glitches are a typical phenomenon of pulsarsprobably all pulsars experience them.

Pulsars are commonly identified with neutron stars; which are characterized by a complex structure compris- ing a core, an intermediate region with superfluid neutrons, and a metallic crust. With a chemical potential of the order of $400 \mathrm{MeV}$, as we have seen, the conditions for color superconductivity in the color-flavor-locked (CFL) or the LOFF scenarios might be reached in the core. Before examining this possibility, however, let us describe the standard explanation of glitches, in the form originated by Anderson and Itoh (1973) and Alpar et al. (1984a). This model is based on the idea that the sudden jumps of $\Omega$ are due to the outward movement of rotational vortices in the neutron superfluid, and the interaction of these vortices with the crust. Crucial ingredients of the model are therefore the existence of a superfluid and a crystal (the metallic crust). This is one of the main reasons for the identification of pulsars with neutron stars, as only neutron stars are thought to have a metallic crust. The LOFF state can be relevant in this context because, if there is a LOFF phase inside the pulsar, the superfluid might interact with the LOFF crystal instead of the crust, thus providing an alternative or complementary mechanism for the glitches. Thus far, there is no developed model for the pinning of the superfluid vortices to the QCD LOFF crystals within compact stars. Therefore we limit our survey to an introduction to the subject, along the lines of Alford (2000), Alford et al. (2001b), and Nardulli (2002c).

Let us consider a compact star whose metallic crust rotates with angular velocity $\boldsymbol{\Omega}$. The superfluid inside the star should not rotate because, in the absence of friction, the crust cannot communicate its rotation to the superfluid component. The velocity of the superfluid is $\mathbf{v}_{s}=\hbar \nabla \Phi / m$, where $\Phi$ is the phase of the superfluid condensate wave function. The consequence of this formula would be $\oint_{\gamma} \mathbf{v}_{s} \cdot d \boldsymbol{\ell}=0$. This would imply the absence of rotation in the superfluid, which, however, does not correspond to the state of minimal energy (for a discussion see Landau et al., 1980). The correct condition is

$$
\oint_{\gamma} \mathbf{v}_{s} \cdot d \ell=2 \pi n \kappa
$$

where $\kappa$ is the quantum of vorticity: $\kappa=\hbar / m$. For Eq. (6.32) to hold, the curve $\gamma$ must wind around a singular point; the integer $n$ is the winding number, which counts the number of times the curve goes around the singular point; the most energetically favorable condition is realized by $n=1$. If $\gamma$ is in a plane, the condition (6.32) holds for any plane and the locus of the singular points is a vortex line. In the absence of rotation there are no vortex lines. The minimal angular velocity $\Omega_{\min }$ for the formation of the first vortex line is

$$
\Omega_{\text {crit }}=\frac{\hbar}{m R^{2}} \ln \frac{R}{a} .
$$

Here we are assuming a cylindrical configuration with radius $R ; a$ is a cutoff of the order of the interatomic distances. When $\Omega$ is increased, the number $N$ of vortex lines per unit area in the superfluid increases according to the formula 


$$
N=\frac{m \Omega}{\pi \hbar}
$$

and one gets, instead of Eq. (6.32),

$$
\oint_{\gamma} d \ell \cdot \mathbf{v}_{s}=N A 2 \pi \kappa
$$

where $A$ is the area encircled by $\gamma$. Eventually the vortex lines tend to fill in the entire space. As a numerical example one can estimate $N$ for the pulsar in the Crab nebula. Here $m=2 m_{N}$ (the condensate is formed by neutral bosons: pairs of neutrons) and $\Omega=\Omega_{\text {pulsar }}$ gives $N \simeq 1.9 \times 10^{5} \mathrm{~cm}^{-2}$ with an average distance between vortex lines $d \sim N^{-1 / 2} \sim 10^{-2} \mathrm{~cm}$. If the vortex line is a straight line, $\mathbf{v}_{\mathbf{s}}$ is perpendicular both to the vortex line and to the radius joining the singular point and the point at which we compute $v_{s}$. At a distance $r$ from the singular point one has

$$
v_{s}=\frac{n \kappa}{r},
$$

as can be immediately seen from Eq. (6.32). More generally,

$$
\mathbf{v}_{s}=\frac{\kappa}{2} \int_{v . l .} \frac{d \ell \wedge \mathbf{R}}{R^{3}},
$$

where $\mathbf{R}$ is the distance vector from the vortex line to the point at which we compute the superfluid velocity.

During the rotation, the vortex lines follow the rotational motion of the vessel, which is clear because they are pinned at the boundary of the superfluid. In particular, for rotations around an axis, the vortex lines are, by symmetry, straight lines parallel to the rotation axis. Their motion imitates the motion of the liquid as a whole and, as a consequence, for the superfluid one can also use the hydrodynamical formula

$$
\mathbf{\Omega}=\frac{1}{2} \boldsymbol{\nabla} \wedge \mathbf{v}_{S},
$$

which, in principle, would be valid only for the normal component of the fluid.

Let now $\nu(r)$ be the number of vortices per unit area at a distance $r$ from the rotation axis; if $\mathbf{v}=\mathbf{v}_{s}$ is the superfluid velocity, one gets

$$
\oint_{\gamma} d \boldsymbol{\ell} \cdot \mathbf{v}=\int_{0}^{r} d \mathbf{S} \cdot \boldsymbol{\nabla} \wedge \mathbf{v}=2 \pi \kappa \int_{0}^{r} 2 \pi r^{\prime} \nu\left(r^{\prime}\right) d r^{\prime} .
$$

We put $k=2 \pi \kappa=h / 2 m_{n}$ and write Eq. (6.39) as

$$
2 \pi r^{2} \Omega(r)=k \int_{0}^{r} 2 \pi r^{\prime} \nu\left(r^{\prime}\right) d r^{\prime},
$$

which implies

$$
k \nu(r)=2 \Omega(r)+r \frac{\partial \Omega}{\partial r} .
$$

Since the total number of vortex lines is conserved, one has

$$
\partial \nu / \partial t+\boldsymbol{\nabla} \cdot\left(\nu \mathbf{v}_{r}\right)=0,
$$

where $\mathbf{v}_{r}$ is the radial component of the superfluid velocity. We write Eq. (6.40) as

$$
2 \pi r^{2} \Omega(r)=k \int_{0}^{r} \nu d S
$$

and take the time derivative, using Eq. (6.42), to get

$$
2 \pi r^{2} \frac{\partial \Omega}{\partial t}=-k \int_{0}^{r} d S \nabla \cdot\left(\nu \mathbf{v}_{r}\right) .
$$

Using the Gauss theorem we get $2 \pi r^{2} \partial \Omega / \partial t$ $=-k 2 \pi r \nu v_{r}$, i.e.,

$$
\frac{\partial \Omega}{\partial t}=-\frac{k \nu v_{r}}{r}=-\left(2 \Omega(r)+r \frac{\partial \Omega}{\partial r}\right) \frac{v_{r}}{r} .
$$

Equation (6.45) shows that the only possibility for the superfluid to change its angular velocity $(\dot{\Omega} \neq 0)$ is by means of a radial motion, i.e., $v_{r} \neq 0$.

Let us now consider a rotating superfluid in contact with rotating normal matter on which an external torque is acting (Alpar et al., 1984a). We denote by $I_{c}$ and $\Omega_{c}$ the moment of inertia and angular velocity of the normal components, which, in a neutron star, includes the crust and possibly other components. The equation of motion of the normal component is

$$
I_{c} \dot{\Omega}_{c}(t)=M_{\text {ext }}+M_{\text {int }} .
$$

Besides the external torque $M_{\text {ext }}$, basically related to the spin down of the pulsar (or the steady accretion in binary pulsars), we have included the internal torque $M_{\text {int }}$ due to interaction with the superfluid,

$$
M_{i n t}=-\int d I_{p} \dot{\Omega}(r, t),
$$

where $d I_{p}$ is the infinitesimal moment of inertia of the superfluid component. Equations (6.45)-(6.47) are the equations of motion for the angular velocities $\Omega$ and $\Omega_{c}$ (superfluid and crust). The two velocities are coupled not only through $M_{\text {int }}$, but also by $v_{r}$ because, as we shall show below, $v_{r}$ depends on the difference $\Omega-\Omega_{c}$. We note again that fundamental to this model is the existence of radial motion, for, if $v_{r}=0$, then $\Omega=$ const, and only $\Omega_{c}$ changes, due to the external torque alone.

In a neutron star, superfluid neutrons (in Cooper pairs) coexist with nuclei in the crust. Also in the crust are superfluid neutrons, but they are characterized by a different (and smaller) $\Delta$. Computing the difference in the free energies between the two phases one obtains the difference of pressures and, consequently, the force per unit length of vortex line. Let $b$ be the average distance between the nuclei; $b$ is also the average distance between two consecutive pinning centers. Let us assume 


$$
2 \xi_{0}<b,
$$

where $\xi_{0}$ is the superconducting coherence length, which also gives the dimension of the vortex core, since $\xi_{0}$ is of the order of the spatial extension of the Cooper pair. The maximum pinning force is obtained when the vortex passes through one layer of the lattice; therefore the maximum force per unit length of vortex line is

$$
f_{p} \simeq \frac{\delta E_{p}}{b \xi},
$$

where

$$
\delta E_{p}=F_{s}-F_{c} \propto \rho \Delta_{s}^{2},
$$

where $F_{s}$ and $F_{c}$ are the free energies of the superfluid neutrons and the nucleons in the crust. $\Delta_{s}$ is the gap for superfluid neutrons, and one can neglect $\Delta_{c}$, the gap of superfluid neutrons in the crust, since $\Delta_{c} \ll \Delta_{s}$. Equation (6.50) implies that neutrons tend to remain in the volume $V$ of the vortex core because they experience a force repelling them from the superconducting phase (if neutron-rich nuclei are present, the repulsion will be less important). Typical values for the pinning energy per nucleus $\delta E_{p}$ at densities $3 \times 10^{13}-1.2 \times 10^{14} \mathrm{~g} / \mathrm{cm}^{3}$ are

$$
\delta E_{p}=1-3 \mathrm{MeV},
$$

while $b=25-50 \mathrm{fm}$ and $\xi_{0}=4-20 \mathrm{fm}$ give

$$
f_{p}=40-1200 \mathrm{MeV}^{3} \text {. }
$$

On the basis of these considerations, let us now sketch a possible mechanism for the formation of glitches (Anderson and Itoh, 1973; Alpar, 1977; Alpar et al., 1984a, 1984b; for further references see below). We consider a rotating neutron star with superfluid neutrons in its interior and a metallic crust, which is a simplified model, but adequate for our purposes. As stressed already, we distinguish between the superfluid velocity $\Omega$ and the crust velocity $\Omega_{c}$. Let us suppose that they are initially equal, as a consequence of pinning. Due to the spinning down of the star, $\Omega_{c}$ decreases; as long as the vortex cores are pinned to the crust lattice, the neutron superfluid cannot spin down, because the radial motion is forbidden. There is therefore a relative velocity of the superfluid with respect to the pinned vortex core because $\Omega>\Omega_{c}$ :

$$
\delta \mathbf{v}=\left(\boldsymbol{\Omega}-\boldsymbol{\Omega}_{c}\right) \wedge \mathbf{r} .
$$

The interaction between the normal matter in the core of the vortex line and the rest of the normal matter (nuclei in the lattice, electrons, etc.) produces a Magnus force per unit length given by

$$
\mathbf{f}=\rho \mathbf{k} \wedge \delta \mathbf{v},
$$

where the direction of $\mathbf{k}$ coincides with the rotation axis and its modulus is equal to the quantum of vorticity. $f$ is the force exerted on the vortex line; as it cannot be larger than $f_{p}$ there is a maximum difference of angular velocity that the system can maintain:

$$
\omega_{c r}=\left(\Omega-\Omega_{c}\right)_{\max }=\frac{f_{p}}{\rho k r}=\frac{E_{p}}{\rho k \xi b} .
$$

If $\omega<\omega_{c r}$ the vortices remain pinned at the lattice sites instead of flowing with the superfluid as they generally do inside it (see discussion above). In contrast, if $\omega$ $>\omega_{c r}$, the hydrodynamical forces arising from the mismatch between the two angular velocities ultimately break the crust and produce the conditions for a glitch. A possible way to get it is by the observation following Eq. (6.45). If a bunch of vortex lines are unpinned and move outwards then Eq. (6.45) implies that the angular velocity (and the angular momentum) of the superfluid decreases, and therefore the angular momentum of the crust increases, which is revealed from outside as a spin up of the star, i.e., a glitch. A numerical analysis would require solving the set of equations (6.45)-(6.47), but this is outside the scope of the present review. ${ }^{9}$ Let us instead discuss the possible role of the LOFF phase in this context. The QCD LOFF phase provides a lattice structure independently of the crust. Therefore it meets one of the two requirements of the model for glitches in pulsars we have outlined above, the other being the presence of a superfluid. The only existing calculations for the inhomogeneous phase in color superconductivity have been performed for the case of two flavors, which, however, in the homogeneous case, does not present superfluidity, since there are no broken global symmetries. Superfluidity is, on the other hand, manifested by the CFL phase of QCD. Therefore a realistic application to QCD superfluid has to wait until a calculation of the LOFF phase with three flavors is completed. For the time being, one can make some order-of-magnitude estimates (Alford et al., 2001b). Let us assume the following choice of parameters: $\Delta_{2 S C}=40 \mathrm{MeV}, \Delta_{L O F F}$ $\approx 8 \mathrm{MeV}$, corresponding to the Fulde-Ferrell state; since $q \approx 1.2 \delta \mu \approx 0.7 \Delta_{2 S C}$, one would get for the average distance between nodal planes $b=\pi /(2|\mathbf{q}|) \approx 9 \mathrm{fm}$ and for the superconducting coherence length $\xi_{0}=6 \mathrm{fm}$. From Eq. (3.28), with $\delta \mu=\delta \mu_{1}$ and an extra factor of 4 to take into account the two flavors and the two colors, we get the free energy per unit volume ${ }^{10} \quad\left|F_{\text {LOFF }}\right|=8$ $\times(10 \mathrm{MeV})^{4}$ and therefore, from Eq. (6.50), the pinning energy of the vortex line is

\footnotetext{
${ }^{9}$ Models differ in the mechanism by which angular momentum is released; instead of moving outwards, for example, vortex lines might break the crust or rearrange it. For reviews see Pines and Alpar (1985); Alpar (1995); and, more recently, Ruderman (1991); Epstein and Baym (1992); Alpar et al. (1993); Link and Epstein (1996); Ruderman et al. (1998).

${ }^{10}$ Using the exact expression instead of Eq. (3.28), which is valid only in the weak-coupling limit, one would get $\left|F_{L O F F}\right|$ $=5 \times(10 \mathrm{MeV})^{4}$.
} 


$$
\delta E_{p}=\left|F_{L O F F}\right| \times b^{3}=6 \mathrm{MeV} .
$$

To get the pinning force we cannot use Eq. (6.49) since Eq. (6.48) does not hold in this case. For an order-ofmagnitude estimate one can use

$$
f_{p} \simeq \frac{\delta E_{p}}{b^{2}}
$$

giving a pinning force per unit length of the vortex of the order of

$$
f_{p} \approx 3 \times 10^{3} \mathrm{MeV}^{3} .
$$

Comparing these numerical values with Eqs. (6.51) and (6.52), one can see that these order-of-magnitude estimates give similar figures, and therefore some of the glitches in neutron stars may be generated well inside the star by vortices related to the LOFF phase of QCD.

As we already stated, these conclusions are tentative because the analysis of the QCD LOFF phase needs to be extended to the three-flavor case; moreover, the true ground state is likely to be different from the FuldeFerrel one-plane-wave structure. Nevertheless, they are encouraging and leave open the possibility that neutron stars might provide another laboratory where the inhomogeneous superconducting phase can be studied. It is useful to note that even in quark stars, in the QCD superconducting LOFF phase, one would get a crystal structure with a lattice characterized by a geometric array in which the gap parameter varies periodically. This would overcome the objection that pulsars cannot be strange stars. This objection is based on the following observation: if strange matter exists there, quark stars should be rather common; however, in absence of metallic crusts, strange stars can hardly develop vortices, at least by the model we have described here. On the contrary, if color superconductivity is able to produce a crystalline structure, it could also give rise to glitches, and the argument in favor of the existence of strange stars would be reinforced.

\section{CONCLUSIONS}

Inhomogeneous crystalline superconductivity was predicted 40 years ago by Larkin, Ovchinnikov, Fulde, and Ferrell, but realistic conditions for its experimental investigation became available only a few years ago. In condensed matter the existence of the LOFF phase, with its characteristic space modulation of the energy gap, still awaits complete confirmation. This is due to the fact that it is indeed a subtle effect. It arises when the Fermi surfaces of the two species participating in the Cooper pairing are different. However, for large separation, pairing is not possible at all and superconductivity disappears altogether. In condensed matter the separation of the Fermi surfaces is obtained by a Zeeman splitting due to an exchange interaction caused by a magnetic field. However, the needed field strength is such as to destroy superconductivity due to diamagnetic effects. As we discussed in this paper, the way to avoid the problem is to use unconventional superconductors such as organic compounds. These materials have a layered structure and therefore, if the magnetic field is parallel to the layers, orbital effects can be controlled.

New opportunities have recently arisen for detecting the LOFF phase in atomic physics (ultracold atomic gases), nuclear physics, and especially quark matter. This last development is a consequence of the recent excitement generated by the study of QCD at high density and low temperature. Inhomogeneous crystalline superconductivity in this context could be generated by the difference in quark chemical potentials induced by weak interactions in the inner core of pulsars. Their main phenomenological effect might be to provide a mechanism for the explanation of glitches in pulsars. If pulsars are neutron stars with a core made up by color superconducting matter, this mechanism would be complementary to the standard models of glitches. If pulsars are strange stars, then the crystalline structure of the condensate would provide the possibility of pinning the superfluid vortices and eventually creating glitches.

This paper has aimed to present a unified formalism to describe the LOFF phase in both condensed and hadronic matter. The simplest way, in our opinion, to describe superconductivity effects, including the LOFF state, is by the effective Lagrangian approach. Since they are based on the general mathematical ground of the renormalization group, effective Lagrangians allow the conditions for this unification. The existence of a common mathematical basis should allow experts from one side to fully appreciate and take advantage of the progresses made on the other. We would be gratified if this paper turned out to be useful to this end.

\section{ACKNOWLEDGMENTS}

One of us (G.N.) would like to thank the CERN Theory Division for the kind hospitality offered during the completion of this paper. We would like to thank R. Gatto for his invaluable help in our mutual work and for reading the manuscript, and $\mathrm{E}$. Fabiano and $\mathrm{M}$. Mannarelli for very pleasant scientific collaboration. We also wish to thank K. Rajagopal for a number of useful discussions on color superconductivity and the LOFF phase. Our thanks are finally due to M. Alford, J. Bowers, M. Ciminale, R. Combescot, and H. Shimahara for useful correspondence on the present review.

\section{APPENDIX A: CALCULATION OF $J$ AND $K$}

We give here an outline of the calculation of the integrals $J$ and $K$ appearing in the Ginzburg-Landau expansion at $T=0$. Using the definition of $J$, Eq. (3.5), and $K$, Eq. (3.6), we have 


$$
\begin{aligned}
& J \equiv J\left(\mathbf{q}_{\mathbf{1}}, \mathbf{q}_{\mathbf{2}}, \mathbf{q}_{\mathbf{3}}, \mathbf{q}_{\mathbf{4}}\right)=+\frac{i g \rho}{2} \int \frac{d \hat{\mathbf{w}}}{4 \pi} \int_{-\delta}^{+\delta} d \xi \int_{-\infty}^{+\infty} \frac{d E}{2 \pi} \prod_{i=1}^{4} f_{i}(E, \delta \mu,\{\mathbf{q}\}) \\
&=+\frac{i g \rho}{2} \int \frac{d \hat{\mathbf{w}}}{4 \pi} \int_{-\delta}^{+\delta} d \xi \int_{-\infty}^{+\infty} \frac{d E}{2 \pi} \prod_{i=1}^{2}\left\{\frac{1}{E+i \epsilon \operatorname{sgn} E+\xi-\delta \mu+2 \mathbf{w} \cdot \mathbf{k}_{\mathbf{i}}} \frac{1}{E+i \epsilon \operatorname{sgn} E-\xi-\delta \mu-2 \mathbf{w} \cdot \boldsymbol{\ell}_{\mathbf{i}}}\right\}, \\
& K=K\left(\mathbf{q}_{\mathbf{1}}, \mathbf{q}_{\mathbf{2}}, \mathbf{q}_{\mathbf{3}}, \mathbf{q}_{\mathbf{4}}, \mathbf{q}_{\mathbf{5}}, \mathbf{q}_{\mathbf{6}}\right)=\frac{i g \rho}{2} \int \frac{d \hat{\mathbf{w}}}{4 \pi} \int_{-\delta}^{+\delta} d \xi \int_{-\infty}^{+\infty} \frac{d E}{2 \pi} \prod_{i=1}^{6} f_{i}(E, \delta \mu,\{\mathbf{q}\}) \\
&=\frac{i g \rho}{2} \int \frac{d \hat{\mathbf{w}}}{4 \pi} \int_{-\delta}^{+\delta} d \xi \int_{-\infty}^{+\infty} \frac{d E}{2 \pi} \prod_{i=1}^{3}\left\{\frac{1}{E+i \epsilon \operatorname{sgn} E+\xi-\delta \mu+2 \mathbf{w} \cdot \mathbf{k}_{\mathbf{i}}} \frac{1}{E+i \epsilon \operatorname{sgn} E-\xi-\delta \mu-2 \mathbf{w} \cdot \ell_{\mathbf{i}}}\right\},
\end{aligned}
$$

where we have defined

$$
\begin{aligned}
& \mathbf{k}_{1}=\mathbf{0}, \quad \mathbf{k}_{2}=\mathbf{q}_{1}-\mathbf{q}_{2}, \quad \mathbf{k}_{3}=\mathbf{q}_{1}-\mathbf{q}_{2}+\mathbf{q}_{3}-\mathbf{q}_{4}, \\
& \ell_{1}=\mathbf{q}_{1}, \quad \ell_{2}=\mathbf{q}_{1}-\mathbf{q}_{2}+\mathbf{q}_{3}, \quad \ell_{3}=\mathbf{q}_{1}-\mathbf{q}_{2}+\mathbf{q}_{3}-\mathbf{q}_{4}+\mathbf{q}_{5},
\end{aligned}
$$

with the conditions $\mathbf{q}_{1}-\mathbf{q}_{2}+\mathbf{q}_{3}-\mathbf{q}_{\mathbf{4}}=0$ and $\mathbf{q}_{1}-\mathbf{q}_{2}+\mathbf{q}_{3}$ $-\mathbf{q}_{\mathbf{4}}+\mathbf{q}_{\mathbf{5}}-\mathbf{q}_{\mathbf{6}}=0$ for $J$ and $K$, respectively. We introduce the Feynman variables $x_{j}, y_{j}(j=1,2$ for $J$ and $j=1,2,3$ for $K$ ) to form the vectors $\mathbf{k}=\sum_{i} x_{i} \mathbf{k}_{\mathbf{i}}$ and $\ell=\Sigma_{i} y_{i} \ell_{i}$. After rotation of the energy integration contour $E \rightarrow i p_{4}$ we get

$$
\begin{aligned}
& \int \frac{d E}{2 \pi} \prod_{i} \frac{1}{E+\xi-\delta \mu+2 \mathbf{w} \cdot \mathbf{k}_{\mathbf{i}}} \\
& =\int \frac{i d p_{4}}{2 \pi} \frac{\delta\left(1-\Sigma x_{k}\right)}{\left[i p_{4}+\xi-\delta \mu+2 \mathbf{w} \cdot \mathbf{k}\right]^{2}} \prod_{n} d x_{n}, \\
& \int \frac{d E}{2 \pi} \prod_{i} \frac{1}{E-\xi-\delta \mu-2 \mathbf{w} \cdot \ell_{\mathbf{i}}} \\
& =\int \frac{i d p_{4}}{2 \pi} \frac{\delta\left(1-\Sigma y_{k}\right)}{\left[i p_{4}-\xi-\delta \mu-2 \mathbf{w} \cdot \ell\right]^{2}} \prod_{n} d y_{n} .
\end{aligned}
$$

Next, we perform the $\xi$ integration by the residues method, and the angular integration. For $J$ the result is

$$
\begin{aligned}
J= & -\frac{i g \rho}{8} \int d p_{4} \epsilon\left(p_{4}\right)\left(\delta \mu-i p_{4}\right) \\
& \times \int\left[\prod_{n=1}^{2} d x_{n} d y_{n}\right] \frac{\delta\left(1-\sum x_{k}\right) \delta\left(1-\sum y_{k}\right)}{\left[\left(\delta \mu-i p_{4}\right)^{2}-v_{F}^{2}|\mathbf{k}-\ell|^{2}\right]^{2}} .
\end{aligned}
$$

After the energy integration, we have

$$
\begin{aligned}
J= & -\frac{g \rho}{8} \int\left[\prod_{n=1}^{2} d x_{n} d y_{n}\right] \delta\left(1-\sum x_{k}\right) \\
& \times \delta\left(1-\sum y_{k}\right) \frac{1}{v_{F}^{2}|\mathbf{k}-\ell|^{2}-\delta \mu^{2}} .
\end{aligned}
$$

This expression is general; we can specialize it to the various crystal structures, as explained in the text.

For $K$ we get, instead of Eq. (A5), the result

$$
\begin{aligned}
K= & -\frac{3 i g \rho}{8} \int d p_{4} \epsilon\left(p_{4}\right) \int\left[\prod_{n=1}^{3} d x_{n} d y_{n}\right] \\
& \times \delta\left(1-\sum x_{k}\right) \delta\left(1-\sum y_{k}\right)\left(\delta \mu-i p_{4}\right) \\
& \times \frac{\left(\delta \mu-i p_{4}\right)^{2}+v_{F}^{2}|\mathbf{k}-\ell|^{2}}{\left[\left(\delta \mu-i p_{4}\right)^{2}-v_{F}^{2}|\mathbf{k}-\ell|^{2}\right]^{4}},
\end{aligned}
$$

which, after energy integration, becomes

$$
\begin{aligned}
K= & -\frac{g \rho}{16} \int\left[\prod_{n=1}^{3} d x_{n} d y_{n}\right] \delta\left(1-\sum x_{k}\right) \\
& \times \delta\left(1-\sum y_{k}\right) \frac{3 \delta \mu^{2}+v_{F}^{2}|\mathbf{k}-\ell|^{2}}{\left[v_{F}^{2}|\mathbf{k}-\ell|^{2}-\delta \mu^{2}\right]^{3}} .
\end{aligned}
$$

\section{APPENDIX B: EXPANSION OF П AROUND THE TRICRITICAL POINT}

Let us consider the expansion of $\Pi(q)$ in $\mathbf{Q}=\mathbf{q} v_{F}$, at finite $T$ and $\mu$, which can be obtained from Eq. (3.4) after introducing the Matsubara frequencies:

$$
\begin{aligned}
\Pi(q)= & -\frac{1}{2} g \rho T \int \frac{d \hat{\mathbf{w}}}{4 \pi} \int_{-\delta}^{+\delta} d \xi \\
& \times \sum_{n=-\infty}^{+\infty} \frac{1}{\left(i \omega_{n}-\delta \mu-\xi-2 \hat{\mathbf{w}} \cdot \mathbf{q} v_{F}\right)\left(i \omega_{n}-\delta \mu+\xi\right)} .
\end{aligned}
$$

Expanding the first denominator in the momentum q we find

$$
\begin{aligned}
\Pi(q)= & \frac{1}{2} g \rho T \sum_{n=-\infty}^{+\infty} \int \frac{d \hat{\mathbf{w}}}{4 \pi} \int_{-\infty}^{+\infty} d \xi \\
& \times \sum_{m=0}^{\infty} \frac{1}{\bar{\omega}_{n}^{2}+\xi^{2}} \frac{\left(2 \hat{\mathbf{w}} \cdot \mathbf{q} v_{F}\right)^{2 m}}{\left(i \bar{\omega}_{n}-\xi\right)^{2 m}},
\end{aligned}
$$


where, as in Eq. (2.70),

$$
\bar{\omega}_{n}=\omega_{n}+i \delta \mu \text {. }
$$

Notice that we have inverted the sum over the Matsubara frequencies with the integration over $\xi$. In this way, as we did for the homogeneous case, we are converting the divergence in $\xi$ into a divergence in the series, which can be treated as before by introducing a cutoff in the sum. Performing the angular integration and the integration over $\xi$ with the help of the integral,

$$
\int_{-\infty}^{+\infty} d \xi \frac{1}{\bar{\omega}_{n}^{2}+\xi^{2}} \frac{1}{\left(i \bar{\omega}_{n}-\xi\right)^{2 m}}=(-1)^{m} \frac{\pi}{2^{2 m} \bar{\omega}_{n}^{2 m+1}},
$$

we get eventually

$$
\Pi(q)=\frac{1}{2} g \pi \rho T \sum_{n=-\infty}^{+\infty} \sum_{m=0}^{\infty} \frac{(-1)^{m}}{2 m+1} \frac{Q^{2 m}}{\bar{\omega}_{n}^{2 m+1}} .
$$

By using the definition of the first term in the grand potential as $1-\Pi(q)$ multiplied by $2 / g$, we easily recover the expression (3.42) for $\widetilde{\alpha}$. Analogously, to get $\widetilde{\beta}$ and $\widetilde{\gamma}$, we expand $J$ [see Eq. (3.5)] and $K$ [see Eq. (3.6)].

\section{REFERENCES}

Abrikosov, A. A., 1957, Zh. Eksp. Teor. Fiz. 32, 1442 [Sov. Phys. JETP 5, 1174 (1957)].

Abrikosov, A. A., L. P. Gor'kov, and I. E. Dzyaloshinski, 1963, Methods of Quantum Field Theory in Statistical Physics (Dover, New York).

Agasian, N. O., B. O. Kerbikov, and V. I. Shevchenko, 1999, Phys. Rep. 320, 131.

Agterberg, D. F., and K. Yang, 2001, J. Phys.: Condens. Matter 13, 9259.

Akhiezer, A. I., A. A. Isayev, S. V. Peletminsky, and A. A. Yatsenko, 1999, Phys. Lett. B 451, 430.

Akhiezer, A. I., V. V. Krasil'nikov, S. V. Peletminsky, and A. A. Yatsenko, 1994, Phys. Rep. 245, 1.

Alexander, J. A. X., T. P. Orlando, D. Rainer, and P. M. Tedrow, 1985, Phys. Rev. B 31, 5811.

Alexander, S., and J. McTague, 1978, Phys. Rev. Lett. 41, 702. Alford, M. G., 2000, in Tokyo 1999, Dynamics of Gauge Fields, Tokyo, Japan, 1999, edited by A. Chodos, C. M. Sommerfield, N. Kitazawa, and H. Minakata (Univ. Acad. Press, Tokyo), p. 227.

Alford, M. G., 2001, Annu. Rev. Nucl. Part. Sci. 51, 131.

Alford, M. G., J. Berges, and K. Rajagopal, 1999, Nucl. Phys. B 558, 219.

Alford, M. G., J. Berges, and K. Rajagopal, 2000, Nucl. Phys. B 571, 269.

Alford, M. G., J. A. Bowers, J. M. Cheyne, and G. A. Cowan, 2003, Phys. Rev. D 67, 054018.

Alford, M. G., J. A. Bowers, and K. Rajagopal, 2001a, J. Phys. G 27, 541.

Alford, M. G., J. A. Bowers, and K. Rajagopal, 2001b, Phys. Rev. D 63, 074016.

Alford, M. G., K. Rajagopal, and F. Wilczek, 1998, Phys. Lett. B 422, 247.

Alford, M. G., K. Rajagopal, and F. Wilczek, 1999, Nucl. Phys. B 537, 443.
Alm, T., B. L. Friman, G. Roepke, and H. Schulz, 1993, Nucl. Phys. A 551, 45.

Alm, T., G. Roepke, and M. Schmidt, 1990, Z. Phys. A 337, 355.

Alm, T., G. Roepke, A. Sedrakian, and F. Weber, 1996, Nucl. Phys. A 406, 491.

Alpar, M. A., 1977, Astrophys. J. 213, 527.

Alpar, M. A., 1995, in The Lives of Neutron Stars, edited by M. A. Alpar, Ü. Kiziloglu, and J. van Paradijs (Kluwer, Boston), p. 185.

Alpar, M. A., P. Anderson, D. Pines, and J. Shaham, 1984a, Astrophys. J. 276, 325.

Alpar, M. A., P. Anderson, D. Pines, and J. Shaham, 1984b, Astrophys. J. 278, 791.

Alpar, M. A., H. F. Chau, K. S. Cheng, and D. Pines, 1993, Astrophys. J. 409, 345.

Anderson, M. H., J. R. Ensher, M. R. Matthews, C. E. Wieman, and E. A. Cornell, 1995, Science 269, 198.

Anderson, P., and N. Itoh, 1973, Nature (London) 256, 25.

Anderson, P. W., 1959, J. Phys. Chem. Solids 11, 26.

Aoi, K., W. Dieterich, and P. Fulde, 1974, Z. Phys. 267, 223.

Aslamazov, L. G., 1969, Sov. Phys. JETP 28, 773.

Bailin, D., and A. Love, 1984, Phys. Rep. 107, 325.

Baldo, M., I. Bombaci, and U. Lombardo, 1992, Phys. Lett. B 283, 8 .

Balicas, L., J. Brooks, K. Storr, S. Uji, M. Tokumoto, H. Tanaka, H. Kobayashi, A. Kobayashi, V. Barzykin, and L. P. Gor'kov, 2001, Phys. Rev. Lett. 87, 067002.

Bardeen, J., L. N. Cooper, and J. R. Schrieffer, 1957, Phys. Rev. 106, 162.

Barrois, B. C., 1977, Nucl. Phys. B 129, 390.

Barzykin, V., and L. P. Gor'kov, 2002, Phys. Rev. Lett. 89, 227002.

Beane, S. R., and P. F. Bedaque, 2000, Phys. Rev. D 62, 117502.

Beane, S. R., P. F. Bedaque, and M. J. Savage, 2000, Phys. Lett. B 483, 131.

Beane, S. R., P. F. Bedaque, and M. J. Savage, 2001, Nucl. Phys. A 688, 931.

Bedaque, P. F., 2002, Nucl. Phys. A 697, 569.

Benfatto, G., and G. Gallavotti, 1990, Phys. Rev. B 42, 9967.

Berges, J., and K. Rajagopal, 1999, Nucl. Phys. B 538, 215.

Bernhard, C., J. L. Tallon, C. Niedermayer, T. Blasius, A. Golnik, E. Brücher, R. K. Kremer, D. R. Noakes, C. E. Stronach, and E. J. Ansaldo, 1999, Phys. Rev. B 59, 14099.

Bowers, J. A., J. Kundu, K. Rajagopal, and E. Shuster, 2001, Phys. Rev. D 64, 014024.

Bowers, J. A., and K. Rajagopal, 2002, Phys. Rev. D 66, 065002.

Bradley, C. C., C. A. Sackett, and R. G. Hulet, 1997, Phys. Rev. Lett. 78, 985.

Brown, W. E., J. T. Liu, and H.-c. Ren, 2000a, Phys. Rev. D 61, 114012.

Brown, W. E., J. T. Liu, and H.-c. Ren, 2000b, Phys. Rev. D 62 , 054013.

Brown, W. E., J. T. Liu, and H.-c. Ren, 2000c, Phys. Rev. D 62, 054016.

Bulaevskii, L. N., 1973, Sov. Phys. JETP 37, 1133.

Bulaevskii, L. N., 1974, Sov. Phys. JETP 38, 634.

Burkhardt, H., and D. Rainer, 1994, Ann. Phys. (Leipzig) 3, 181.

Buzdin, A. I., and H. Kachkachi, 1997, Phys. Lett. A 225, 341. Buzdin, A. I., and M. L. Kulic, 1984, J. Low Temp. Phys. 54, 203. 
Buzdin, A. I., and S. V. Polonski, 1987, Zh. Eksp. Teor. Fiz. 93, 747 [Sov. Phys. JETP 66, 422 (1987)].

Buzdin, A. I., and V. V. Tugushev, 1983, Zh. Eksp. Teor. Fiz. 85, 735 [Sov. Phys. JETP 58, 428 (1983)].

Carter, G. W., and D. Diakonov, 1999, Phys. Rev. D 60, 016004. Casalbuoni, R., in QCD@Work: International Workshop on Quantum Chromodynamics: Theory and Experiment, Bari, Italy, 2001, edited by P. Colangelo and G. Nardulli, AIP Conference Proceedings No. 602 (AIP, New York), p. 358.

Casalbuoni, R., A. Deandrea, N. Di Bartolomeo, R. Gatto, F. Feruglio, and G. Nardulli, 1997, Phys. Rep. 281, 145.

Casalbuoni, R., F. De Fazio, R. Gatto, G. Nardulli, and M. Ruggieri, 2002, Phys. Lett. B 547, 229.

Casalbuoni, R., Z. Duan, and F. Sannino, 2000, Phys. Rev. D 62, 094004.

Casalbuoni, R., E. Fabiano, R. Gatto, M. Mannarelli, and G. Nardulli, 2002, Phys. Rev. D 66, 094006.

Casalbuoni, R., and R. Gatto, 1999, Phys. Lett. B 464, 111.

Casalbuoni, R., R. Gatto, M. Mannarelli, and G. Nardulli, 2001, Phys. Lett. B 511, 218.

Casalbuoni, R., R. Gatto, M. Mannarelli, and G. Nardulli, 2002a, Phys. Rev. D 66, 014006.

Casalbuoni, R., R. Gatto, M. Mannarelli, and G. Nardulli, 2002b, Phys. Lett. B 524, 144.

Casalbuoni, R., R. Gatto, and G. Nardulli, 2001, Phys. Lett. B 498, 179.

Casalbuoni, R., R. Gatto, and G. Nardulli, 2002, Phys. Lett. B $\mathbf{5 4 3}, 139$.

Casalbuoni, R., R. Gatto, G. Nardulli, and M. Ruggieri, 2003, Phys. Rev. D 68, 0302077.

Chandrasekhar, B. S., 1962, Appl. Phys. Lett. 1, 7.

Clogston, A. M., 1962, Phys. Rev. Lett. 9, 266.

Collins, J. C., and M. J. Perry, 1975, Phys. Rev. Lett. 34, 1353.

Combescot, R., 2001, Europhys. Lett. 55, 150.

Combescot, R., and C. Mora, 2002, Eur. Phys. J B 28, 397.

Cooper, L. N., 1956, Phys. Rev. 104, 1189.

Davis, K. B., M.-O. Mewes, M. R. Andrews, N. J. van Druten, D. S. Durfee, D. M. Kurn, and W. Ketterle, 1995, Phys. Rev. Lett. 75, 3969.

Decroux, M., and Ø. Fischer, 1982, in Superconductivity in Ternary Compounds, Part II, edited by M. B. Maple and $\varnothing$. Fischer (Springer, Heidelberg), p. 57.

DeMarco, B., and D. S. Jin, 1999, Science 285, 1703.

DeMarco, B., S. B. Papp, and D. S. Jin, 2001, Phys. Rev. Lett. 86, 5409.

Deryagin, D. V., D. Y. Grigoriev, and V. A. Rubakov, 1992, Int. J. Mod. Phys. A 7, 659.

Dupuis, N., 1995, Phys. Rev. B 51, 9074.

Dupuis, N., G. Montambaux, and C. A. R. Sá de Melo, 1993, Phys. Rev. Lett. 70, 2613.

Dyugaev, A. M., I. D. Vagner, and P. Wyder, 2001, JETP Lett. 73, 411.

Eichten, E., and B. Hill, 1990, Phys. Lett. B 234, 511.

Eilenberger, G., 1968, Z. Phys. 214, 195.

Epstein, R. I., and G. Baym, 1992, Astrophys. J. 387, 276.

Evans, N., J. Hormuzdiar, S. D. H. Hsu, and M. Schwetz, 2000, Nucl. Phys. B 581, 391.

Evans, N., S. D. H. Hsu, and M. Schwetz, 1999a, Nucl. Phys. B 551, 275.

Evans, N., S. D. H. Hsu, and M. Schwetz, 1999b, Phys. Lett. B 449, 281.
Frautschi, S. C., 1978, in Proceedings of the Workshop on Hadronic Matter at Extreme Energy Density, Erice, Italy, 1978, edited by N. Cabibbo and L. Sertorio (Plenum, New York), p. 18.

Fried, D. G., T. C. Killian, L. Willmann, D. Landhuis, S. C. Moss, D. Kleppner, and T. J. Greytak, 1998, Phys. Rev. Lett. 81, 3811.

Fulde, P., and R. A. Ferrell, 1964, Phys. Rev. 135, A550.

Gegenwart, P., M. Deppe, M. Koppen, F. Kromer, M. Lang, R. Modler, M. Weiden, C. Geibel, F. Steglich, T. Fukase, and N. Toyota, 1996, Ann. Phys. (N.Y.) 5, 307.

Georgi, H., 1990, Phys. Lett. B 240, 447.

Giannakis, I., J. T. Liu, and H.-c. Ren, 2002, Phys. Rev. D 66, 031501.

Ginzburg, L., and L. D. Landau, 1950, Zh. Eksp. Teor. Fiz. 20, 1064.

Ginzburg, V. L., 1957, Sov. Phys. JETP 4, 153.

Gloos, K., R. Modler, H. Schimanski, C. D. Bredl, C. Geibel, F. Steglich, A. I. Buzdin, N. Sato, and T. Komatsubara, 1993, Phys. Rev. Lett. 70, 501.

Goddard, P., S. Tozer, J. Singleton, A. Ardavan, A. Abate, and

M. Kurmoo, 2002, J. Phys.: Condens. Matter 14, L 495.

Goodman, A. L., 1999, Phys. Rev. C 60, 014311.

Gor'kov, L. P., 1959, Zh. Eksp. Teor. Fiz. 36, 1918 [Sov. Phys. JETP 9, 1364 (1959)].

Gor'kov, L. P., 1960, Sov. Phys. JETP 10, 593.

Gor'kov, L. P., and A. G. Lebed', 1987, Europhys. Lett. 4, 941.

Granade, S. R., M. E. Gehm, K. M. O'Hara, and J. E. Thomas, 2002, Phys. Rev. Lett. 88, 120405.

Gross, D., and F. Wilczek, 1973, Phys. Rev. Lett. 30, 1343.

Gruenberg, L. W., and L. Gunther, 1966, Phys. Rev. Lett. 16, 996.

Gubankova, E., W. V. Liu, and F. Wilczek, 2003, Phys. Rev. Lett. 91, 032001.

Hadjiev, V. G., A. Fainstein, P. Etchegoin, H. J. Trodahl, C. Bernhard, M. Cardona, and J. L. Tallon, 1999, Phys. Status Solidi B 211, R5.

Hong, D. K., 2000a, Nucl. Phys. B 582, 451.

Hong, D. K., 2000b, Phys. Lett. B 473, 118.

Hong, D. K., 2001, Acta Phys. Pol. B 32, 1253.

Hong, D. K., V. A. Miransky, I. A. Shovkovy, and L. C. R. Wijewardhana, 2000, Phys. Rev. D 61, 056001.

Houzet, M., and A. I. Buzdin, 2000, Europhys. Lett. 50, 375.

Houzet, M., A. I. Buzdin, L. Bulaevskii, and M. Maley, 2002, Phys. Rev. Lett. 88, 227001.

Houzet, M., Y. Meurdesoif, O. Coste, and A. I. Buzdin, 1999, Physica C 316, 89.

Hsu, S. D. H., 2000, in Tokyo 1999, Dynamics of Gauge Fields, Tokyo, Japan, 1999, edited by A. Chodos, C. H. Sommerfield, N. Kitazawa, and H. Minakata (Univ. Acad. Press, Tokyo), p. 247.

Hsu, S. D. H., and M. Schwetz, 2000, Nucl. Phys. B 572, 211.

Huxley, A. D., C. Paulson, O. Laborde, J. L. Tholence, D. Sanchez, A. Junod, and R. Calemczuk, 1993, J. Phys.: Condens. Matter 5, 7709.

Isayev, A. A., 2002, Phys. Rev. C 65, 031302.

Isgur, N., and M. B. Wise, 1989, Phys. Lett. B 232, 113.

Isgur, N., and M. B. Wise, 1990, Phys. Lett. B 237, 527.

Ishiguro, T., 2000, J. Supercond. 13, 817.

Jaccarino, V., and M. Peter, 1962, Phys. Rev. Lett. 9, 290.

Kamerlingh-Onnes, H., 1911, Commun. Phys. Lab. Univ. Leiden 120b, 122b, 124c. 
Klein, U., D. Rainer, and H. Shimahara, 2000, J. Low Temp. Phys. 118, 91.

Krey, U., 1973, Int. J. Magn. 4, 153.

Kundu, J., and K. Rajagopal, 2002, Phys. Rev. D 65, 094022.

Landau, L., and E. M. Lifshitz, 1996, Statistical Physics, Part I (Butterworth-Heinemann, London).

Landau, L., E. M. Lifshitz, and L. P. Pitaevskii, 1980, Statistical Physics, Part II (Pergamon, Oxford).

Larkin, A. J., and Y. N. Ovchinnikov, 1964, Zh. Eksp. Teor. Fiz. 47, 1136 [Sov. Phys. JETP 20, 762 (1965)].

Le Bellac, M., 1996, Thermal Field Theory (Cambridge University, Cambridge, England).

Lebed', A. G., 1986, JETP Lett. 44, 114.

Leibovich, A. K., K. Rajagopal, and E. Shuster, 2001, Phys. Rev. D 64, 094005.

Link, B., and R. I. Epstein, 1996, Astrophys. J. 457, 844.

Liu, W. V., and F. Wilczek, 2003, Phys. Rev. Lett. 90, 047002.

London, F., and H. London, 1935, Proc. R. Soc. London, Ser. A 149, 71 .

Machida, K., and H. Nakanishi, 1989, Phys. Rev. B 30, 122.

Maki, K., 1964, Physics (Long Island City, N.Y.) 1, 127.

Maki, K., and H. Won, 1996, Czech. J. Phys. 46, 1035.

Manalo, S., and U. Klein, 2000, J. Phys.: Condens. Matter 28, L471.

Manohar, A. V., and M. B. Wise, 2000, Heavy Quark Physics, Cambridge Monographs on Particle Physics, Nuclear Physics, and Cosmology No. 10 (Cambridge University, Cambridge, England).

Martinez-Pinedo, G., K. Langanke, and P. Vogel, 1999, Nucl. Phys. A 651, 379.

Matsuo, S., S. Higashitani, Y. Nagato, and K. Nagai, 1998, J. Phys. Soc. Jpn. 67, 280.

Meissner, W., and R. Ochsenfeld, 1933, Naturwissenschaften 21, 787.

Mewes, M. O., G. Ferrari, F. Schreck, A. Sinatra, and C. Salomon, 1999, Phys. Rev. A 61, 011403.

Modler, R., P. Gegenwart, M. Lang, M. Deppe, M. Weiden, T. Lühmann, C. Geibel, F. Steglich, C. Paulsen, J. L. Tholence, N. Sato, T. Komatsubara, Y. Onuki, M. Tachiki, and S. Takahashi, 1996, Phys. Rev. Lett. 76, 1292.

Modugno, G., G. Roati, F. Riboli, F. Ferlaino, F. J. Brech, and M. Inguscio, 2002, Science 297, 2240.

Murthy, G., and R. Shankar, 1995, J. Phys.: Condens. Matter 7, 9155.

Muther, H., and A. Sedrakian, 2002, Phys. Rev. Lett. 88, 252503.

Nam, M.-S., J. Symington, J. A. Singleton, S. J. Blundel, A. Ardavan, M. Kurmoo, and P. Day, 1999, J. Phys.: Condens. Matter 11, L477.

Nambu, Y., 1960, Phys. Rev. 117, 648.

Nambu, Y., and G. Jona-Lasinio, 1961a, Phys. Rev. 122, 345.

Nambu, Y., and G. Jona-Lasinio, 1961b, Phys. Rev. 124, 246.

Nardulli, G., 2002a, Riv. Nuovo Cimento 25N3, 1.

Nardulli, G., 2002b, paper delivered at Compact Stars in the QCD Phase Diagram, Copenhagen. Available from conference website http://www.slac.stanford.edu/econf/C010815/ proceedings.html

Nardulli, G., 2002c, in Quark-Gluon Plasma and Heavy Ion Collisions, Frascati, Italy, 2002, edited by W. M. Alberico, M. Nardi, and M. P. Lombardo (World Scientific, Singapore), p. 216.

Neubert, M., 1994, Phys. Rep. 245, 259.

Norman, M. R., 1993, Phys. Rev. Lett. 71, 3391.
O'Hara, K. M., M. E. Gehm, S. R. Granade, S. Bali, and J. E. Thomas, 2001, Phys. Rev. Lett. 85, 2092.

Park, B.-Y., M. Rho, A. Wirzba, and I. Zahed, 2000, Phys. Rev. D 62, 034015.

Pickett, W. E., R. Weht, and A. B. Shick, 1999, Phys. Rev. Lett. 83, 3713.

Pines, D., and A. Alpar, 1985, Nature (London) 316, 27.

Pisarski, R. D., and D. H. Rischke, 1999a, Phys. Rev. Lett. 83, 37.

Pisarski, R. D., and D. H. Rischke, 1999b, Phys. Rev. D 60, 094013.

Pisarski, R. D., and D. H. Rischke, 2000a, Phys. Rev. D 61, 051501.

Pisarski, R. D., and D. H. Rischke, 2000b, Phys. Rev. D 61, 074017.

Polchinski, J., 1993, in Recent Directions in Particle Theory: From Superstrings and Black Holes to the Standard Model (TASI-92), Boulder, Colorado, 1992, edited by J. Harvey and J. Polchinski (World Scientific, River Edge, NJ), p. 235.

Politzer, H. D., 1973, Phys. Rev. Lett. 30, 1346.

Pringle, D. J., J. L. Tallon, B. G. Walker, and H. J. Trodahl, 1999, Phys. Rev. B 59, R11 679.

Rajagopal, K., 1999, Nucl. Phys. A 661, 150.

Rajagopal, K., 2001, in QCD@Work: International Workshop on Quantum Chromodynamics: Theory and Experiment, Bari, Italy, 2001, edited by P. Colangelo and G. Nardulli, AIP Conference Proceedings No. 602 (AIP, New York), p. 339.

Rajagopal, K., and E. Shuster, 2000, Phys. Rev. D 62, 085007. Rajagopal, K., and F. Wilczek, 2001, in At the Frontier of Particle Physics, edited by M. Shifman (World Scientific, Singapore), Vol. 3, p. 2061.

Rapp, R., T. Schafer, E. V. Shuryak, and M. Velkovsky, 1998, Phys. Rev. Lett. 81, 53.

Rapp, R., T. Schafer, E. V. Shuryak, and M. Velkovsky, 2000, Ann. Phys. (N.Y.) 280, 35.

Rapp, R., E. V. Shuryak, and I. Zahed, 2001, Phys. Rev. D 63, 034008.

Rauchschwalbe, U., W. Lieke, C. D. Bredl, F. Steglich, J. Aarts, K. M. Martini, and A. C. Mota, 1982, Phys. Rev. Lett. 49, 1448.

Rischke, D. H., D. T. Son, and M. A. Stephanov, 2001, Phys. Rev. Lett. 87, 062001.

Roati, G., F. Riboli, G. Modugno, and M. Inguscio, 2002, Phys. Rev. Lett. 89, 140403.

Ruderman, M., 1991, Astrophys. J. 382, 587.

Ruderman, M., T. Zhu, and K. Chen, 1998, Astrophys. J. 492, 267.

Saint-James, D., G. Sarma, and E. J. Thomas, 1969, Type II Superconductivity (Pergamon, Oxford, England).

Samohkin, K. V., 1997, Physica C 274, 156.

Sarma, G., 1963, J. Phys. Chem. Solids 24, 1029.

Schafer, T., 2000, Nucl. Phys. B 575, 269.

Schafer, T., 2001, Int. J. Mod. Phys. B 15, 1474.

Schafer, T., and F. Wilczek, 1999a, Phys. Rev. Lett. 82, 3956.

Schafer, T., and F. Wilczek, 1999b, Phys. Lett. B 450, 325.

Schafer, T., and F. Wilczek, 1999c, Phys. Rev. D 60, 074014.

Schafer, T., and F. Wilczek, 1999d, Phys. Rev. D 60, 114033.

Schimanski, H., K. Gloos, F. Martin, R. Modler, C. Geibel, C. Schank, and F. Steglich, 1994, Physica B 199, 125.

Schreck, F., L. Khaykovich, K. L. Corwin, G. Ferrari, T. Bourdel, and J. Cubizolles, 2001, Phys. Rev. Lett. 87, 080403.

Sedrakian, A., 2001, Phys. Rev. C 63, 025801. 
Sedrakian, A., T. Alm, and U. Lombardo, 1997, Phys. Rev. C 55, R582.

Sedrakian, A., and U. Lombardo, 2000, Phys. Rev. Lett. 84, 602.

Shankar, R., 1994, Rev. Mod. Phys. 66, 129.

Shimahara, H., 1994, Phys. Rev. B 50, 12760.

Shimahara, H., 1997, J. Phys. Soc. Jpn. 66, 541.

Shimahara, H., 1998a, J. Phys. Soc. Jpn. 67, 736.

Shimahara, H., 1998b, J. Phys. Soc. Jpn. 67, 1872.

Shimahara, H., 2002, J. Phys. Soc. Jpn. 71, 1644.

Shimahara, H., S. Matsuo, and K. Nagai, 1996, Phys. Rev. B 53, 12284.

Shimahara, H., and D. Rainer, 1997, J. Phys. Soc. Jpn. 66, 3591. Shovkovy, I. A., and L. C. R. Wijewardhana, 1999, Phys. Lett. B 470, 189.

Shuster, E., and D. T. Son, 2000, Nucl. Phys. B 573, 434.

Singleton, J., N. Harrison, C. H. Mielke, J. Schlueter, and A. M. Kini, 2001, J. Phys.: Condens. Matter 13, L899.

Singleton, J., J. A. Symington, M.-S. Nam, A. Ardavan, M. Kurmoo, and P. Day, 2000, J. Phys.: Condens. Matter 12, L641.

Son, D. T., 1999, Phys. Rev. D 59, 094019.

Son, D. T., and M. A. Stephanov, 2001, Phys. Rev. Lett. 86, 592. Splittorff, K., D. T. Son, and M. A. Stephanov, 2001, Phys. Rev. D 64, 016003.
Symington, J. A., J. Singleton, M.-S. Nam, A. Ardavan, M. Kurmoo, and P. Day, 2001, Physica B 294-295, 418.

Takada, S., 1970, Prog. Theor. Phys. 43, 27.

Takada, S., and T. Izuyama, 1969, Prog. Theor. Phys. 41, 635.

Tallon, J., C. Bernhard, M. Bowden, P. Gilbert, T. Stoto, and D. Pringle, 1999, IEEE Trans. Appl. Supercond. 9, 1696.

Tanatar, M. A., T. Ishiguro, H. Tanaka, and H. Kobayashi, 2002, Phys. Rev. B 66, 134503.

Tenya, K., S. Yasunami, T. Tayama, H. Amitsuka, T. Sakakibara, M. Hedo, Y. Inada, Y. Huga, E. Yamamoto, and Y. Ōnuki, 1999, Physica B 259-261, 692.

Thomas, F., B. Wand, T. Luhmann, P. Gegenwart, G. R. Stewart, F. Steglich, J. P. Brison, A. Buzdin, L. Glemot, and J. Flouquet, 1996, J. Low Temp. Phys. 102, 117.

Uji, S., H. Shinagawa, T. Terashima, T. Yakabe, Y. Terai, M. Tokumoto, A. Kobayashi, H. Tanaka, and H. Kobayashi, 2001, Nature (London) 410, 908.

Vonderfecht, B. E., C. G. Gearhart, W. H. Dickhoff, A. Polls, and A. Ramos, 1991, Phys. Lett. B 253, 1.

Werthamer, N. R., H. Helfand, and P. C. Hohenberg, 1966, Phys. Rev. 147, 295.

Wilczek, F., 2000, Nucl. Phys. A 663, 257.

Won, H., and K. Maki, 2002, Physica B 322, 315.

Yang, K., 2001, Phys. Rev. B 63, 140511.

Yang, K., and D. F. Agterberg, 2000, Phys. Rev. Lett. 84, 4970. Yin, G., and K. Maki, 1993, Phys. Rev. B 48, 650. 